IvBSIR 81-2264

\title{
Models for the Migration of Low Molecular Weight Additives in Polyolefins
}

L. E. Smith, S. S. Chang, F. L. McCrackin

G. A. Senich and F. W. Wang

Polymer Science and Standards Division

Center for Materials Science

U.S. Department of Commerce

National Bureau of Standards

Washington, DC 20234

Annual Report for the Period

October 1, 1979 - September 30, 1980

Issued April 1981

Prepared for

Bureau of Foods

QC Food and Drug Administration

100 Washington, DC 20201 

MODELS FOR THE MIGRATION OF LOW MOLECULAR WEIGHT ADDITIVES IN POLYOLEFINS

L. E. Smith, S. S. Chang, F. L. McCrackin

G. A. Senich and F. W. Wang

Polymer Science and Standards Division

Center for Materials Science

U.S. Department of Commerce

National Bureau of Standards

Washington, DC 20234

Annual Report for the Period

October 1, 1979 - September 30, 1980

Issued April 1981

Prepared for

Bureau of Foods

Food and Drug Administration

Washington, DC 20201

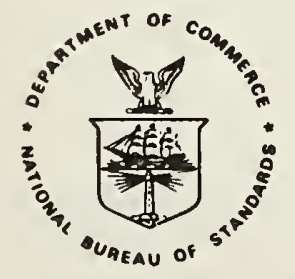

U.S. DEPARTMENT OF COMMERCE, Malcolm Baldrige, Secretary NATIONAL BUREAU OF STANDARDS, Ernest Ambler, Director 
$\ldots+\cdots, 8=0$

(5)

;

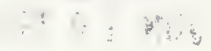


Abstract

Food Packaging is an important encounter in the daily life. The low molecular weight components in the packaging materials may migrate into the foods. This program, sponsored by the Bureau of Foods of the Food and Drug Administration, is to provide theoretical models, reliable data base, and methodology to study the migration phenomena and to provide reasonable worst-case estimates for the concentrations of the indirect additives in food. In this annual report we present a relationship of diffusion coefficients of gaseous diffusants in polyolefins based on free volume theory, procedures and results of inverse gas chromatography for migrant-polymer interaction parameters and diffusivities of oligomers in polyethylene, methods and preliminary results of spectrofluorimetry on the migration of antioxidants in polyethylene, results and correlations of extraction experiments with radioactive labeled migrants. It was found that anhydrous ethanol and lower members of pure triglycerides can successfully simulate the extractive behavior of food oils. The accelerating action of $n$-heptane over that of the food oil is quantified.

Key Words: Antioxidants, Diffusion, Food Packaging, Spectrofluorimetry, Inverse Gas Chromatography, Migration, $0 i l$ Simulants, 01 igomers, Radioactive Tracer, Polyolefins 

I. An Empirical Relationship Between the Diffusion Coefficient

in Hydrocarbon Polymers and Its Interpretation by

Fractional Free Volume.

II. Activation Energies for Diffusion in Polyolefins.

III. IGC Measurement of the Polymer-Migrant Interaction Parameter . . .

IV. IGC Measurement of Polymer-Migrant Diffusion Coefficients

Theory . . . . . . . . . . . . IV

Experiment and Calculations .......... IV-7

Gas Phase Contributions . . . . . . . . . IV-8

Polymer Phase Contributions ............. IV-11

Concentration Effects .................. IV-17

Capillary Columns ..................... IV-18

Adsorption Effects...................... IV-18

Other IGC Studies of Diffusion in Polymers ......... IV-19

Conclusions and Future Work ............ IV-21

V. Fluorescence Measurements of Antioxidant Migration from

Plastics.................. V 1

Introduction ................ . . . . $v-1$

Experimental ....................... $V-1$

Results and Discussion................... $V-6$

VI. Experimental Measurement of Migration by Extraction . . . . . VI-1

Introduction ................ . . VI-1

Experimental Run Designation............... VI-5

Estimation of Diffusion Coefficients . . . . . . . . VI-6

Results of Extraction Experiments ........... VI-10

Typical Examples of Extraction Experiments ........ VI-11

Correlation of Diffusion Coefficients .......... . VI-12

Effect of Temperature ................... VI-13

Effect of Solvent ...................... VI-14

Effect of Different Polyolefins ............. VI-16

Effect of Migrants ................. VI-19

Effect of Migrant Concentrations ............ . VI-20

Summary of Conclusions ................. VI-21 



\section{Introduction}

The daily encounters of food packaging materials become an important aspect of life as more foods are sold in packaged form for the purpose of preservation and ease of distribution. The Bureau of Foods of the Food and Drug Administration has regulatory responsibility of the use of packaging materials in contact with food. Any substances that migrate from the food packaging materials into foods are considered to be indirect food additives. The regulatory decisions on the types of packaging materials to be used on various types of foods are based on the indirect food additives that can be expected to migrate from the package into the food, its toxicological knowledge and expected amount of migration during storage and usage. Since it is impossible to test every conceivable case of combinations of polymer-migrant-food, the program at the National Bureau of Standards, sponsored by the Food and Drug Administration, is aimed to provide sound technical assistance and reliable data to the Food and Drug Administration and to recommend theoretical or empirical estimation and extrapolation methods to yield at least a reasonable worst-case estimates for regulatory purposes. However, this report represents only the technical opinions of the authors as representatives of the National Bureau of Standards and should not be taken in any way as regulatory decisions or recommendations of the Bureau of Foods.

In this report we present an empirical relationship of diffusion coefficients of gaseous diffusants and polymers based on free volume theory, procedures and results of inverse gas chromatography and spectrofluorimetry, results and correlations of extraction experiments with radioactive labeled migrants.

An empirical relationship between the zero concentration diffusion coefficient of gases in polyolefins, similar to the one presented in a previous report, has been interpreted by means of free volume theory. 
Inverse gas chromatography has been used to generate the specific retention volume $\mathrm{V}_{\mathrm{g}}{ }^{\circ}$ and then to estimate the polymer-migrant interaction parameter $x^{\infty}$ needed to calculate the partition coefficients from solution theory. Relative ranking of diffusivities of probe molecules in the polymer phase above the polymer glass transition temperature or in the liquid phase can also be estimated from inverse gas chromatographic method.

A procedure for using spectro-fluorimeter as a selective and sensitive method detection of migrants, especially antioxidants, have been developed and presented in this report together with preliminary results on the migration of $N, N^{\prime}$-diphenyl-p-phenylenediamene from low density polyethylene into $n$-heptane.

In the past year we have substantially completed a large number of tests involving the migration of radioactive ${ }^{14} \mathrm{C}$-labeled straight chain paraffinic oligomers (n-octadecane and n-dotriacontane) and an antioxidant (butylatedhydroxytoluene) from two different polyethylenes (high density or linear polyethylene and low density or branched polyethylene) and isotactic polypropylene into various solvents including a variety of triglycerides, alcohols, hydrocarbons and water at temperatures from room temperature to $60^{\circ} \mathrm{C}$. Among the correlations for various parameters, it was found that either the pure triglycerides, such as tributyrin and trioctanoin, or anhydrous ethanol may successfully be employed as an oil simulant in all cases studied. The accelerating action of $n$-heptane over that of the food oil is quantified.

Future works in the additive migration program would be mainly in the area of migration from glassy polymers, where the diffusion coefficients are many orders of magnitude slower than that in the amorphous or semi-crystalline polymers above their glass transition temperatures. 


\section{SECTION I}

An Empirical Relation for Diffusion of Gases in Hydrocarbon Polymers and in Interpretation in Terms of Fractional Free Volume 

The zero concentration diffusion coefficient, D, of gases in polyolefins', (polyethylenes of different crystallinity and natural rubber) shows a remarkable regularity. Not only is the order of the gases according to $D$ the same for all polyolefins, but the diffusion coefficient of a gas in a polymer is given in terms of its diffusion coefficient, $D_{r}$, in natural rubber by

$$
D=K D_{r}^{m}
$$

where the values of $K$ and $m$ depend only on the polyolefin and not on the gas. For natural rubber, $K$ and ii must equal 1. Experimental diffusion coefficients at $25^{\circ} \mathrm{C}$ of eleven vapors in three polyethylene films and in natural rubber are given in table 1 and plotted on $\log -\log$ scales in figure 1 . The points lie on straight lines, justifying equation (1). The relative standard deviation of the fit of the diffusion coefficients to equation ( 1 ) is 9.5 percent. This compares favorably with the wide variation of $D$ which varies by a factor of 1000 .

The empirical rule formulated in equation (1) can be derived on the basis of the fractional free volume concept of the transport properties of polymers. In a rough approximation, the zero concentration diffusion coefficient of a gas in a polymer depends, according to Fujita ${ }^{2}$, on the fractional free volume $f$ of the polymer by

$$
D=A e^{-B / f}
$$


Table 1. Diffusion Coefficients $0 \times 10^{8} \mathrm{~cm}^{2} / \mathrm{s}$, at $25^{\circ} \mathrm{C}$ in Natural Rubber $\left(\alpha_{v}=0\right)$ and Three Polyethylenes and The Corrected Value $D_{r a}$ in Natural Rubber.

\begin{tabular}{|c|c|c|c|c|c|}
\hline & $\alpha_{y}$ & Vol ume & Crystal & iity & \\
\hline Gas & 0 & 0.29 & 0.43 & 0.77 & $D_{r a}$ \\
\hline $\mathrm{O}_{2}$ & 173 & 120 & 46 & 17 & 169 \\
\hline A & 136 & 96 & 36 & 11.6 & 136 \\
\hline $\mathrm{CO}_{2}$ & $125^{7^{2}}$ & 97 & $37 . \overline{2}$ & 12.4 & 134 \\
\hline CO & 135 & 82 & 33.2 & 9.6 & 126 \\
\hline $\mathrm{N}_{2}$ & 117 & 74 & 32 & 9.3 & 117 \\
\hline $\mathrm{CH}_{4}$ & 89 & 54 & 19.3 & 5.7 & 86.1 \\
\hline $\mathrm{CH}_{3} \cdot \mathrm{CH}_{3}$ & 40 & 24 & 6.8 & 1.46 & 39.5 \\
\hline $\mathrm{CH}_{2}: \mathrm{C}: \mathrm{CH}_{2}$ & 50 & 31 & 10.5 & 2.5 & $52^{\circ}$ \\
\hline $\mathrm{CH}_{3} \cdot \mathrm{CH}: \mathrm{CH}_{2}$ & 31 & 20 & 5.8 & 1.1 & 33.1 \\
\hline $\mathrm{CH}_{3} \cdot \mathrm{CH}_{2} \cdot \mathrm{CH}_{3}$ & 21 & 12 & 3.22 & 0.49 & 21.2 \\
\hline $\mathrm{SF}_{6}$ & 11.5 & 5.6 & 1.35 & 0.16 & 11.1 \\
\hline
\end{tabular}




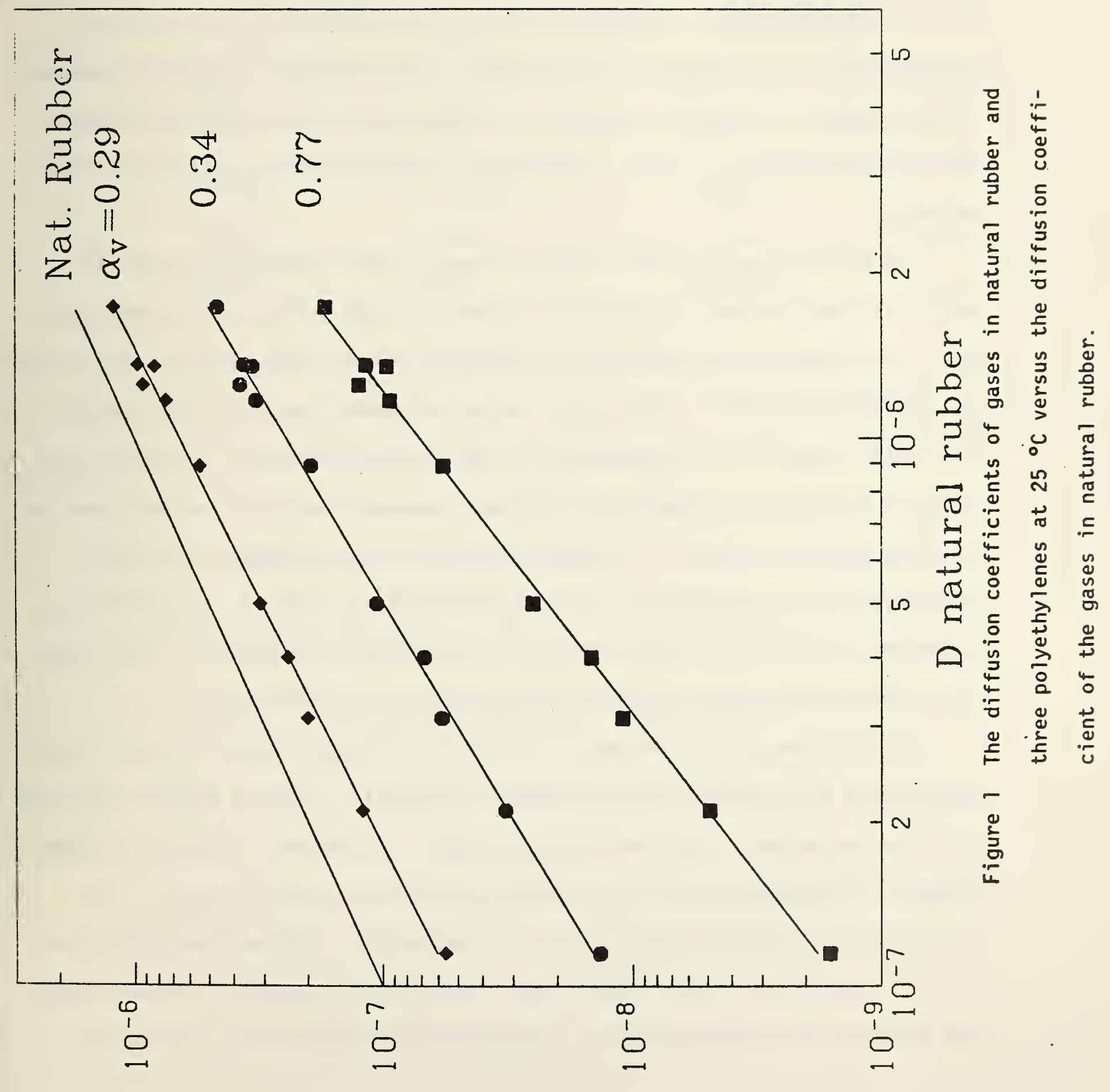


as long as the whole interaction of the gas and polymer can be taken into account by $f$ which is a characteristic property of the polymer at the temperature of the experiment. $B$ depends on the size and shape of the diffusing molecule but is independent of the polymer. The factor $A$ is certainly dependent on the polymer. According to Fujita, A also depends on the size and shape of the diffusing molecule, but is independent of the temperature of the diffusing molecule.

The four polymers in this study are very similar chemically and differ mainly in their volume crystallinity; shown in table 2 which varies from 0 to 0.77. The crystallinity affects the diffusion coefficients mainly by the detour and blocking factors 3,4 . The detour factor decreases the diffusivity because the crystal lamellae are impermeable to the diffusing molecule so that it must travel a longer path through the amorphous component than would be the case for a purely amorphous material. Because the detour factor depends only on the supercrystalline morphology of the sample, it does not depend on the diffusing molecule or the fraction free volume of the amorphous component of the polymer. Thus, the detour factor only affects the factor $A$ in equation (2).

The blocking factor reduces the diffusivity because some of the more narrow passages in the amorphous layers between the parallel lamellae become impassable to larger molecules. Thus the blocking factor is dependent on both the polymer through its morphology and on the diffusing molecule through its size. The blocking factor affects both $A$ and $f$ in equation (2). Because the size of the diffusing molecules used in this study, from $0_{2}$ to $\mathrm{SF}_{6}$, covers a limited range, the effect of the blocking factor on the diffusion coefficients is assumed to 
Table 2. $K, m$ and Volume Crystallinity, $\alpha_{v}$ for Three Polyethylenes and Natural Rubber $\left(\alpha_{v}=0\right)$ at $25^{\circ} \mathrm{C}$.

$\begin{array}{lcl}\frac{\alpha}{v} & k & m \\ 0 . & \bar{i} & \frac{j}{1} \\ 0.29 & 2.791 & 1.105 \\ 0.43 & 18.72 & 1.314 \\ 0.77 & 1326 . & 1.712\end{array}$


be small; $A$ is therefore assumed to depend only on the polymer. This automatically excludes very small $\left(\mathrm{H}_{2}, \mathrm{He}\right)$ and very large penetrant molecules.

With these assumptions, equation (1) is derived from equation (2). Let $A_{r}$ and $f_{r}$ be the values of $A$ and $f$ for natural rubber. Then by equation (2),

$$
D_{r}=A_{r} e^{-B / f} r
$$

Eliminating $B$ from equations (2) and (3) gives

$$
D=\left(A / A_{r}{ }^{f} / f\right) D_{r} f / f
$$

Equation (4) is identical to equation (1) if one defines

$$
\begin{aligned}
& m=f_{r} / f \\
& K=A / A_{r}^{m}
\end{aligned}
$$

with both $m$ and $K$ dependent on the polymer but independent of the diffusing molecule.

The fractional free volume of the amorphous phase of a polymer is given by f. Therefore, if the amorphous material in the semi-crystalline polymers were unaltered by the crystals, its fraction free volume $f$ would be equal to that of natural rubber, $f_{r}$ and the value of $m$ would be one by equation (5). In fact, the fractional free volume of the amorphous phase is seen to be reduced by the crystals; for the polyethylene as a volume crystallinity of 0.77 , one obtains

$$
f=0.584 f_{r}
$$


i.e., a little more than half the fractional free volume in the fully amcrptivunatural rubber. Figure 2 shows the values of $f / f_{r}$ for the polymers investigated.

The polymer molecules in the amorphous phase of a semi-crystalline polymer are more constrained than those of a completely amorphous polymer, so that the fractional free volume is expected to be less. However, its reduction by almost a factor of two is surprising.

Equation (1) was found to fail for diffusion coefficients of hydrogen and helium. This may be understood from the derivation of equation (1). The blocking factor was assumed to be independent of the diffusing molecule. This assumption is not valid for hydrogen or helium which are very small and can diffuse in many channels in the polymer that block larger molecules.

A similar consideration can be applied to the $B / f$ term in equation (2). If the interactions between the diffusing molecules and the polymer vary with the diffusing molecules, the fractional free volume of the polymer does not adequately describe this interaction. Then the value of $B$ depends on this interaction so that it also depends on the polymer instead of depending only on the diffusing molecule. Because all of the polymers investigated were chemically similar, the interaction of a particular molecule is nearly the same in all of the polymers.

One of the sources of the derivation between the experimental values of $D$ and the values computed by equation ( 1 ) is experimental errors of $D_{r}$, the diffusion coefficients of the gases in natural rubber. Therefore, the values of $D_{r}$ were adjusted to improve the fit. The adjusted values, equation (1) fit the diffusion coefficients with a relative standard error of 4.7 percent. The values of $k$ and $m$ are shown in table 2 . 


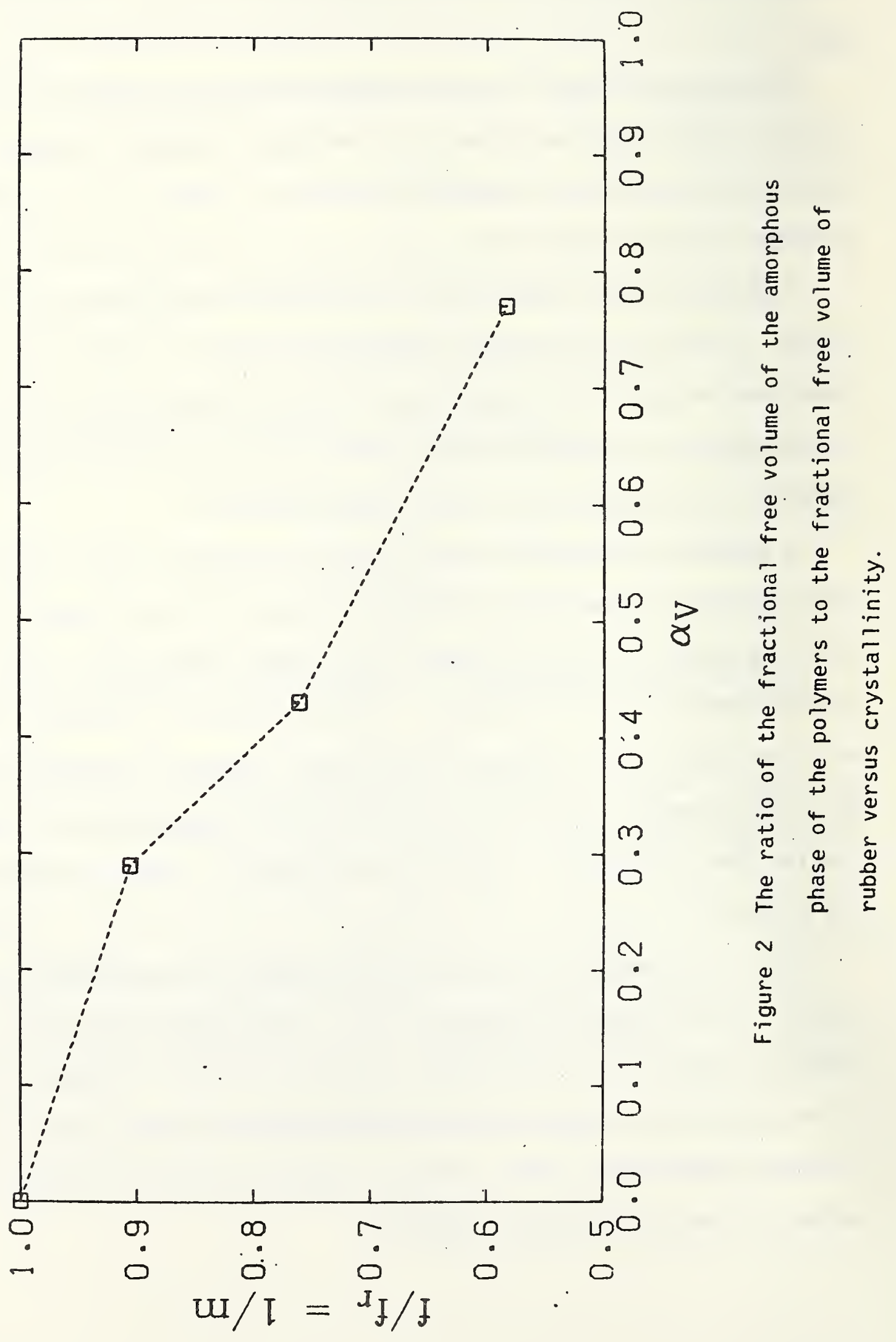




\section{References}

1. A. S. Michaels and H. J. Bixler, J. Polym. Sci., 50, 413 (1961).

2. H. Fujita, Adv. Polymer Sci., 3, 1 (1961).

3. C. H. Klute, J. Appl. Polym. Sci., I, 340 (1959).

4. A. S. Michaels and R. B. Parker, Jr., J. Polym. Sci., 4l, 53 (1959).

5. A. Peterlin and F. L. McCrackin, J. P. Sym. Sci., Physics, in press. 



\section{SECTION II}

Activation Energies for Diffusion in Polyolefins 
. 
Diffusion coefficients and activation energies for diffusion of manv migrants in polyolefins have been collected by Flynn. The activation energies for high and low density polyethylene given by Flynn as well as those given in the Polymer Handbook are shown in Figure 1 versus the molecular weight of the migrant. Despite the large scatter, the activation energies in high and low density polyethylene are seen to be the same, and to increase with increasing molecular weight of the migrant.

Figure 2 shows the activation energies of five other polyolefins. The line is the same $l$ ine as in Figure 1 and gives the average value for polyethylene. The increase in the activation energies with the molecular weight of the migrant is again observed. The activation energies of migration in isotactic polypropylene and polyisbutylene are seen to be larger than in polyethylene, while the activation energies in stereoblock polypropylene and hydrogenated polybutadiene are seen to be larger than in polyethylene. The data for poly(4methylpentene-4) are not sufficient to draw any conclusions.

The scatter in these curves are not unexpected. Many experimental measurements of the activation energies are based on few measurements of the diffusion coefficients so they are inaccurate. Also, the activation energy in a given polymer depends on the chemical structure and shape of a migrant in addition to the molecular weight of the migrant. However, these figures can still provide the best estimate of the activation energy for diffusion of a migrant in a polyolefin when no other information is available. 


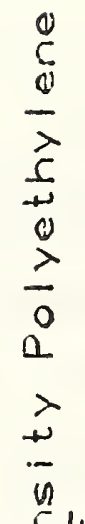

5 L.i.

$\stackrel{0}{\circ} \alpha$ $3+>$

0.

0 C

co 0

ᄃ. $\frac{5}{0} 3$

- I

$c+\square$

.

5

in

4 .

$\overline{0}$

4

(I)
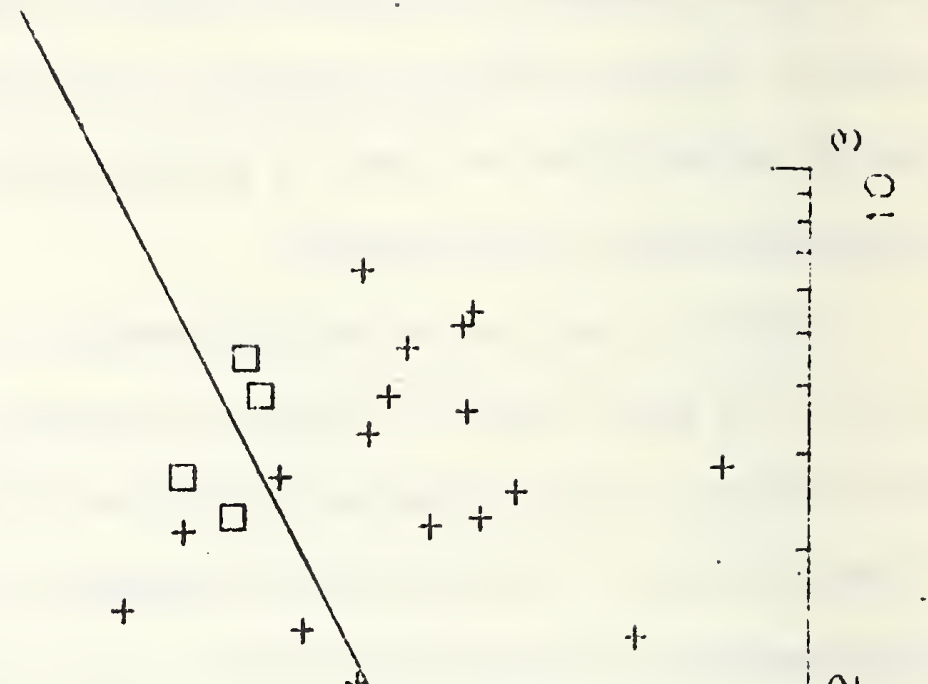

5

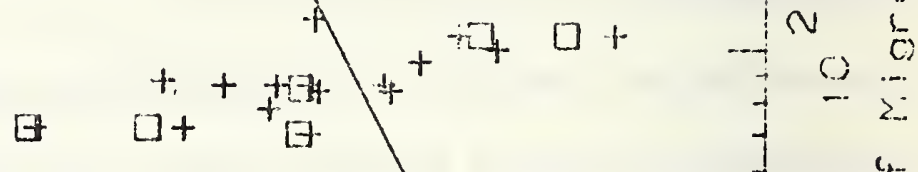

$+$
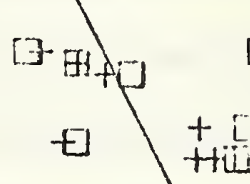

b

juiti 
\begin{tabular}{lll}
0 & 0 \\
0 & 0 & 1 \\
\hline & 0 & 0
\end{tabular}

每入

И

$-0 \geqslant$

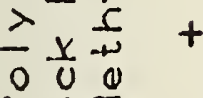

$00 \Sigma$

- $\bar{\Omega} \frac{1}{\sigma}$

$4 \frac{1}{0}$

$0+0$

○口

$\begin{array}{lll}n & 0 \\ 4 & 0 & 0\end{array}$

4 (1)

$\div-7$

$0+$

ᄂ. 03

$4 . \Omega$

$\lambda=\frac{\lambda}{0}$

o) 0 a

ᄂ

500

c 0

1
0

4 0

$\begin{array}{lll}0 & 0 & 1 \\ > & 0 & 0\end{array}$

$-1>$

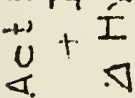
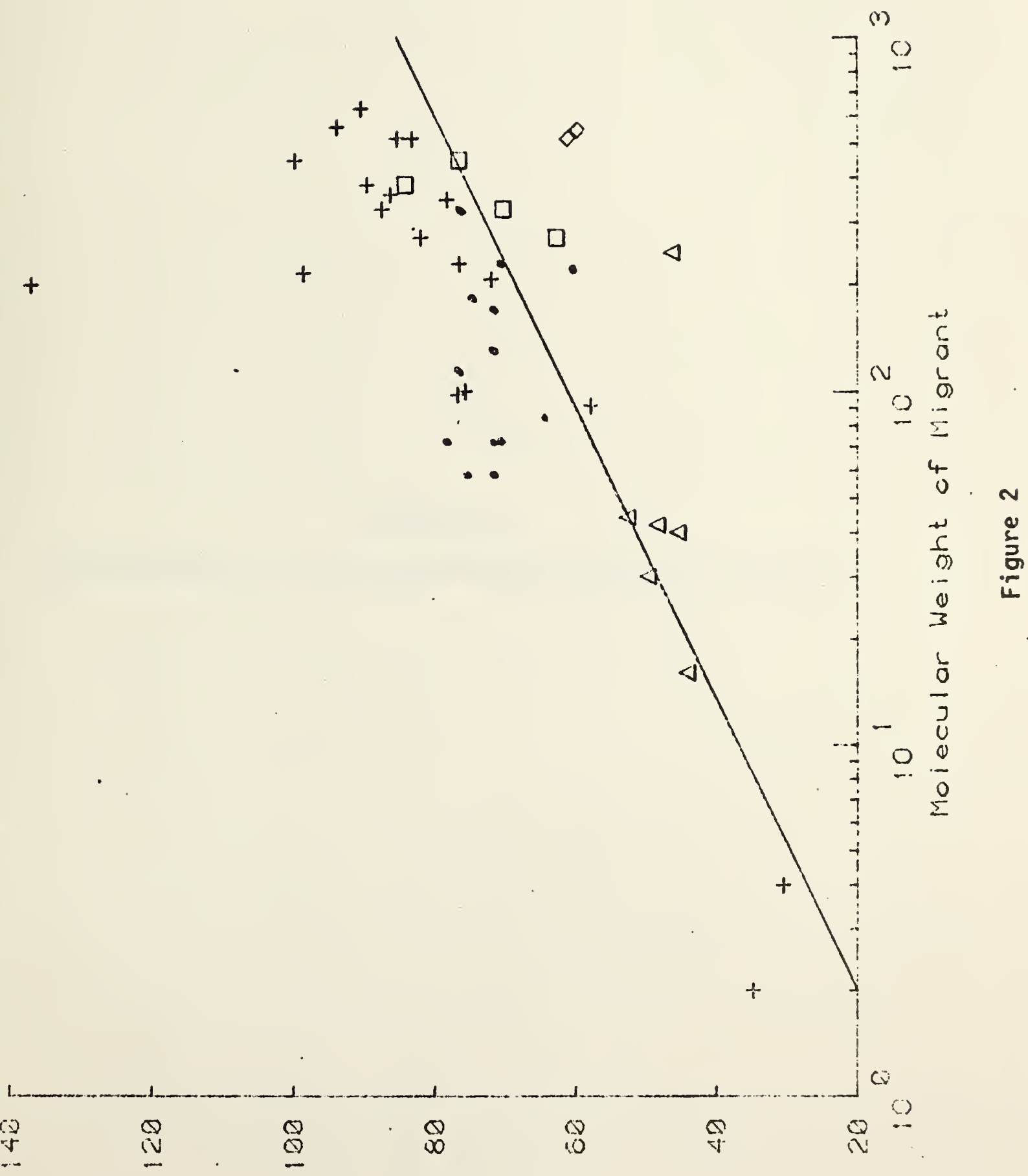

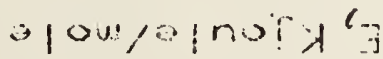


. 
SECTION III

IGC Measurement of the Polymer-Migrant Interaction Parameter 

The polymer-migrant interaction parameter, $x$, can be determined experimentally from the results of osmotic pressure, swelling, sorption, vapor pressure, and heat of mixing studies. Such studies are often conducted at relatively high migrant concentrations. This necessitates extrapolation to zero migrant concentration in order to estimate the interaction parameter $x^{\infty}$ needed to calculate the partition coefficient describing the distribution of a migrant between the solvent and polymer phases. Inverse gas chromatography (IGC) provides a method for the determination of $x^{\infty}$ at migrant or probe levels so low that extrapolation to zero concentration may not be required. The specific retention volume, $V_{g}^{0}$, is the IGC result from which $x^{\text {w }}$ is calculated as previously outlined in NBSIR 80-1999. In order to investigate the capabilities and limitations of this method, we have determined both $v_{g}^{0}$ and $x^{\infty}$ for several migrants in the linear polyethylene SRM 1475 at temperatures above and below the polymer melting point. The results for decane-SRM 1475 and an analysis of the source of errors encountered in this typical determination are given in the previously cited NBS report. Determination of the total mass of polymer in the column makes a significant contribution to the overall uncertainty in $v_{g}^{0}$. We have found that this quantity can be obtained more reliably from gravimetric determinations on a calcined diatomite or glass bead support than by solvent refluxing treatments. When these methods are applied our results for decane-SRM 1475 are identical, within the limits of uncertainty, to literature data available for similar systems.

The $X^{\infty}$ parameter can be calculated from an IGC determined $V_{g}^{0}$ with the aid of a theory which describes the behavior of polymer solutions. These theories provide a link between $V_{g}^{0}$ and $x$ by relating $x^{\infty}$ to the weight fraction activity coefficient of the probe. The latter quantity is readily calculated from the experimentally determined $V_{g}^{0}$. 
Several theoretical treatments are possible but all lead to a similar relation between $x^{\infty}$ and $v_{g}^{0}$. The accuracy of $\chi^{\infty}$ depends on the uncertainties in $v_{g}^{0}$ and input parameters needed for the solutions theories. The latter are difficult to ascertain, however, and errors in $x^{\infty}$ have not been estimated in our work. The agreement between values calculated from the Flory equation-of-state theory and the Sanchez and Lacombe lattice fluid based treatment of polymer solutions is generally good.

A large body of IGC-determined polymer-solvent interaction parameters is available. Table $I$ is a compendium of references to $x$ values derived from major IGC studies on a variety of polymer-solvent systems. It should be noted that all the studies cited have been conducted at temperatures above the polymer glass transition. Values of $x^{\infty}$ derived from IGC experiments are in good agreement with those obtained from other methods of measurement, as discussed in two interlaboratory comparisons $(1,2)$.

Specific retention volumes and interaction parameters determined below the melting point of a semicrystalline polymer have a greater degree of uncertainty than the corresponding results obtained from the molten polymer. The crystalline material present below $T_{m}$ does not contribute to bulk sorption; therefore, the effective mass of polymer in the column is that of the amorphous fraction. As the crystalline content of a semicrystalline polymer is a nominal value that can be determined to an accuracy of about $5 \%$ at best, this additional uncertainty will contribute to the error in the effective mass of polymer in the column and be reflected in the experimental $v_{g}^{0}$ and the $x^{\infty}$ value derived therefrom.

\section{References}

1. W. E. Hammers and C. L. DeLigny, J. Polym. Sci., Phys., 12, 2065 (1974).

2. Y.-K. Leung and B. E. Eichinger, J. Phys. Chem., 78, 60 (1974). 
TABLE 1

Literature Sources of IGC $\times$ Values

\begin{tabular}{|c|c|c|}
\hline Polymer $^{\mathrm{a}}$ & Probe Type $^{b}$ & Reference \\
\hline Linear polyethylene & $A, B, D, E$ & 1 \\
\hline Branched polyethylene & $A, B, C, D, E, F$ & $1,2,14$ \\
\hline Polyethyl ene copolymers & $A, C, D, E, F$ & $2,3,14$ \\
\hline Polypropylene & $A, C, D, E, F$ & 3,14 \\
\hline Poly(vinyl acetate) & $A, C, D, E$ & 4 \\
\hline Poly(vinyl methyl ether) & $A, C, D, F$ & 5 \\
\hline Poly(vinyl chloride) & $A, F$ & 6 \\
\hline Poly( $\varepsilon$-caprol actone $)$ & $A, F$ & 6 \\
\hline Polystyrene & $A, B, C, D, E, F$ & $5,7,8,9$ \\
\hline Poly(methyl methacrylate) & $A, B, D, E$ & 9 \\
\hline Polyisoprene & $A, B, \dot{D}, E$ & 3,10 \\
\hline Polyisobutylene & $A$ & 11 \\
\hline Polysiloxanes & $A, B, D, E$ & 12,13 \\
\hline \multicolumn{3}{|c|}{$a_{\text {all }}$ measurements reported are above the polymer $T_{g}$} \\
\hline \multicolumn{3}{|c|}{$\begin{aligned}{ }^{b}=\text { normal alkane } & B=\text { branched alkane } C=\text { chlorinated al kane } \\
D=\text { aromatic } & E=\text { branched aromatic } F=\text { compounds containing } \\
& \text { heter atomis }\end{aligned}$} \\
\hline
\end{tabular}

1. H. P. Schreiber, Y. B. Tewari, and D. Patterson, J. Polym. Sci. Phys., 11, 15 (1973).

2. R. D. Newman and J. M. Prausnitz, AIChE J., 19, 704 (1973).

3. K. Ito and J. E. Guillet, Macromol., 12, 1163 (1979).

4. D. D. Deshpande and 0. S. Tyagi, Macromol., 11, 746 (1978).

5. C. S. Su and D. Patterson, Macromol., 10, 708 (1977).

6. 0. Olabisi, Macromol., 8 , 316 (1975).

7. M. Galin and M. C. Rupprecht, Polymer, 19, 506 (1978).

8. F. H. Covitz and J. W. King, J. Polym. Sci. A-1, 10, 689 (1972).

9. G. DiPaola-Baranyi and 1. F. Guillet, Macromol., 11, 228 (1978).

10. Y. B. Tewari and H. P. Schreiber, Macromol., 5, 329 (1972).

11. Y.-K. Leung and B. E. Eichinger, J. Phys. Chem., 78, 60 (1974).

12. H. R. Summers, Y. B. Tewari, and H. P. Schreiber, Macromol., 5, 12 (1972).

13. M. Galin, Macromol., 10, 1239 (1977).

14. G. DiPaola-Baranyi, J. E. Guillet, H.-E. Jeberien, and J. Klein, Macromol. Chem., 181, 215 (1980). 


\section{.}


SECTION IV

IGC Measurements of Polymer-Migrant Diffusion Coefficients 
THEORY

The nrobe or migrant injected onto a chromatographic column resides partly in the carrier gas and partially in the stationary phase during transit through the column. Diffusion of the probe vapor in the gas phase can occur parallel to the background flow of carrier gas sweeping the vapor through the column both with and opposed to the carrier flow direction. This longitudinal gaseous diffusion process, that is, diffusion in the gas phase along the carrier flow direction through the column, causes an increase in width of the eluted peak over its initial injection distribution. An additional gas phase peak spreading mechanism originates in the multiplicity of path lengths possible around the packed support particles and leads to an associated broadened distribution of elution times. Peak spreading can also be attributed to diffusive processes in the stationary phase. If the carrier gas flow rate is very rapid, equilibrium partitioning of the vapor between the gas and stationary phases cannot be established. The residence time in the stationary liquid phase is influenced by both the diffusivity and the distance in the stationary phase over which diffusion must occur, among other factors. Longitudinal diffusion in the liquid phase, however, has been found to be insignificant compared to peak spreading arising from the corresponding gas phase process and can be ignored in all cases. Peak broadening is commonly quantified by the construct of the height equivalent to a theoretical plate $H$, related to the width at half-height $w_{1 / 2}$ for an eluted gaussian-shaped peak as follows:

$$
H=\frac{\sigma^{2}}{L}=\frac{L}{5.54}\left(\frac{w_{1 / 2}}{t_{R}}\right)^{2}
$$

where $L$ is the column length, $\sigma$ the standard deviation, and $t_{R}$ the retention time of the peak at the maximum.

An equation attributed to van Deemter et al. (3) is often used to relate $H$ to the carrier gas flow velocity $u$ and is of the form: 


$$
H=A+B / U+C u
$$

where the A term arises from the number of possible gas paths through the column, $B$ from longitudinal diffusion of the probe in the carrier gas, and $C$ from the resistance to mass transfer in the 1iquid or stationary phase. The coefficients in equation 2 are defined as follows:

$$
\begin{aligned}
& A=2 \lambda d_{p} \\
& B=2 \gamma D_{g} \\
& C=\frac{8 d_{f}^{2} k}{\pi^{2} D_{\ell}(1+k)^{2}}
\end{aligned}
$$

where $\lambda$ is a measure of support packing irregularities, $d_{p}$ the average support particle diameter, $\gamma$ a correction for the tortuosity of the gas flow in the column, $D_{g}$ the probe diffusivity in the gas phase, $d_{f}$ the effective thickness of the liquid phase film, $D_{\ell}$ the diffusivity of the probe in the liquid phase, and $k$ the column capacity factor commonly given as:

$$
k=\frac{t_{R}-t_{M}}{t_{M}}
$$

$t_{M}$ being the time taken to elute an unsorbed material, such as methane or air, from the column. Equation 2 indicates that at high flow rates $H$ will be proportional to $u$ as illustrated in Figure 1. The probe-liquid diffusion coefficient can then be determined from the constant of proportionality $C$ by Equation 5 if the film thickness is known. Equation 5 is applicable for diffusion into a spherical particle. A more commonly encountered situation is diffusion into a uniform stationary phase film on a planar solid surface which leads to a similar relation for $\mathrm{C}$ :

$$
C=\frac{2 d_{f}^{2} k}{3 D_{\ell}(1+k)^{2}}
$$

While equation 2 gives a suitable explanation of peak spreading in some cases, other results have raised questions about its utility. Bohemen and 


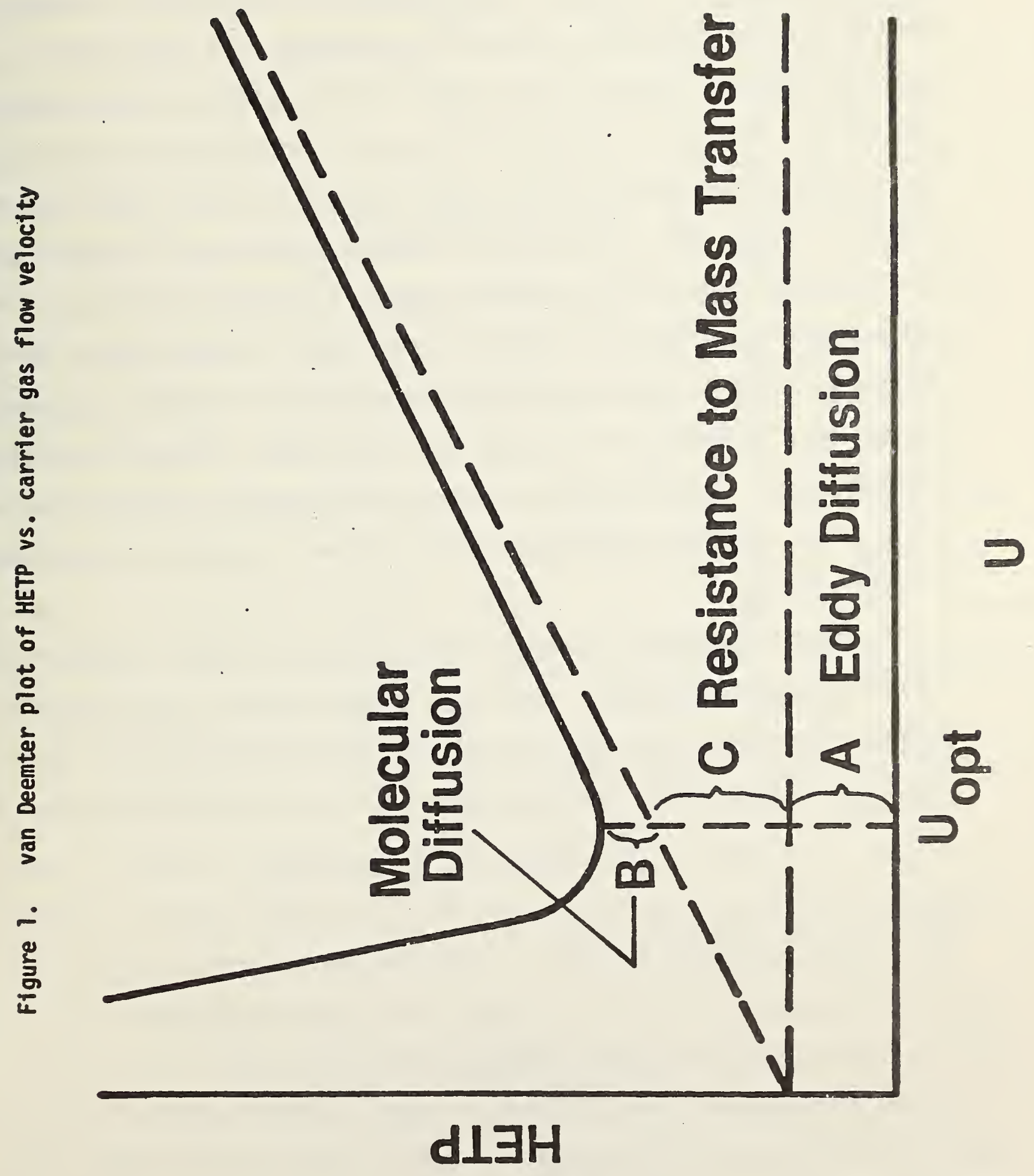


Purnell (4) determined the coefficients in equation 2 for several systems and found $A$ values which were unexpectedly independent of the particle size and sometimes negative, a result not predictable by the van Deemter treatment. Giddings (5) has developed a theory which postulated that the $A$ term is velocity dependent, having a zero value at low flow rate and reaching the 1 imiting value of equation 3 at high velocity. A serious question to this prediction was raised by experiments with air samples where there is no sorption by the stationary phase $(k=0)$. The expected asymptotic approach to a limiting value of A at high flow rates was not realized; rather, a minimum in the $H$ vs. $u$ plot was observed in all cases as typified by the work of Kieselbach (6). These discrepancies indicate that the simplifications made by van Deemter in neglecting other mechanisms of diffusion are not valid for all systems. Giddings and coworkers (7) examined several modified forms of the van Deemter equation and found that none could account for the experimental results obtained on columns of varying length and outlet pressure.

An equation proposed by Jones (8) takes several additional sources of peak spreading into consideration: resistance to mass transfer in the gas phase normal to the carrier gas flow direction (D), velocity distribution effects (E), and a correlation between these two processes ( $F)$. This equation is given as:

$$
H=A+B / U+(C+D+E+F) u
$$

where $C$ is as in equation 7 and

$$
\begin{aligned}
& D=\frac{C_{D} k^{2} d_{g}^{2}}{D_{g}(1+k)^{2}} \\
& E=\frac{C_{E} d_{p}^{2}}{D_{g}} \\
& F=\frac{2 \theta\left(C_{D} C_{E}\right)^{T / 2} k d_{p} d_{g}}{D_{g}(1+k)}
\end{aligned}
$$


$d_{g}$ being the diffusion path length in the gas, $\theta$ the correlation coefficierit between the $D$ and $E$ terms, and $C_{D}$ and $C_{E}$ geometric constants. The $D$ term was discounted by van Deemter in the derivation of equation 2 because of the much greater magnitude of $D_{g}$ compared to $D_{\ell}$. This simplification is valid for the thick liquid films used in their work, studies of columns with roughty 0.3 mass fraction stationary phase loading. If low column loadings which reduce $d_{f}$ to about $0.01 \mathrm{~d}_{\mathrm{g}}$ are used, then the expected difference in diffusivity between liquid and gas phases is overcome and the $D$ term cannot be ignored. In addition, if the support surface is not completely covered then $C_{D}$ must increase by a factor of the average number of times for diffusion to the wall before contact with the liquid phase is made. The functional form of the $D$ term given in equation 9 is not correct since its plate height contribution is predicted to approach zero as the vapor becomes increasingly insoluble in the liquid phase. This difficulty has been examined by Purnell (9) who concludes that the discrepancy is a consequence of neglecting the nonuniform velocity profile. For an infinite value of $k$, equation 9 is expected to give a result about an order of magnitude less than the theoretical limiting $C_{D}$ value of 0.115 derived for an ideal distillation process.

The $E$ contribution to spreading arises from diffusion caused by differences in the velocity of the carrier gas in various parts of the column. When the support is porous the carrier gas in the interior of the particles is relatively stagnant. The time taken by the probe vapor to diffuse from the particle interior to the flowing carrier stream contributes to the peak broadening process. The diffusion distance from the particle interior to the fast flowing carrier stream is on the order of the particle radius. Other velocity differences can arise from short and long range channels formed by support packing irregularities and transcolumn effects caused by the influence of the tube walls and coiling of the column. These effects are summarized by Giddings (10) who estimates approximate magnitudes for contributions of the many possible velocity gradients combined in the $E$ term of equation 8. 
While the first three terms in equation 8 are independent of one another, the $D$ and $E$ terms are not since molecules which have a lower average velocity will spend a smaller fraction of time in the gas phase and therefore not experience as much gaseous diffusion as molecules with higher than average velocity. The total variance of two dependent contributors to the variance is given as:

$$
\sigma_{i j}^{2}=\sigma_{i}^{2}+\sigma_{j}^{2}+2 \theta_{i j} \sigma_{i} \sigma_{j}
$$

The $F$ term of equation 11 originates from the final term in the above equation and corresponds to the interaction between the $D$ and $E$ terms.

Giddings (11) has given an expression simitar to equation 3 which accounts for all known plate height terms contributing at least $1 \%$ of the total plate height for packed GC columns in the absence of interfacial adsorption as:

$$
H=\sum_{i} \frac{1}{1 / A_{i}+T / E_{j} u}+\frac{B}{u}+\sum_{i} C_{i} u+D u+H_{t c}
$$

The $H_{t c}$ term represents the contribution of column-wide velocity inequality effects, such as that arising from column coiling, and could alternatively be included in $E$. If the $A$ term is small, a situation of ten encountered at normal operating velocities, it can be neglected and the gaseous diffusion $D$ and $E$ terms combined. A compilation of the $C, D$, and $E$ coefficients expected to apply in various situations and a means of calculating their magnitude from measurable column parameters is given in reference 10.

The plate height equations discussed to this point apply only to the peak spreading in a localized area of the chromatographic column. A final modification should be made to equations 8 and 13 to account for the variation in local carrier velocity along the column caused by the drop in pressure from column inlet to outlet. The effect of this correction is primarily on the liquid mass transfer term, as discussed by Da] Nogare and Juvet (12). The final expression for the 
experimentally accessible column average plate height $\bar{H}$ dependence on the carrier gas outlet velocity $u_{0}$ is similar to equation 8 and is given as:

$$
\bar{H}=A+\frac{B}{u_{0}} \quad\left(\frac{2 p_{0} C}{p_{i}+p_{0}}+D+E+F\right) u_{0}
$$

where $p_{i}$ and $p_{0}$ are the column inlet and outlet pressure. The gas phase diffusivity found in equations $4,9,10$, and 11 must be stated at the outlet pressure of the column. The form of this modification is based on the relationship between the local column velocity and the average carrier gas velocity. The preceeding treatment assumes a distance average of this velocity. Other methods (7) involve the use of a pressure average velocity and yield a slightly different equation for $\bar{H}$ but agree reasonably well with equation 14 even at large values of the inlet pressure.

The previous description of the theory of peak broadening in packed gas chromatographic columns is complicated by the ill-defined nature of packing regularity, stationary phase geometry, and gas flow paths in the column. Such variables necessitate the introduction of empirical geometry or flow coefficients into the equations describing peak spreading. This limitation is overcome when the geometrically simple open tubular column is employed. Golay (13) has obtained an exact solution for the case of peak broadening arising from a capillary column with a uniform liquid phase coating on the inner wall of the tube and a parabolic carrier gas flow velocity distribution. In this instance $\bar{H}$ is given by the equation:

$$
\bar{H}=\frac{2 D_{g}^{0}}{u_{0}}+\frac{1+6 k+11 k^{2}}{24(1+k)^{2}}+\frac{r^{2} u_{0}}{D_{g}^{0}}+\frac{2 k}{3(1+k)^{2}} \frac{d^{2} u}{D_{l}}
$$

where $r$ is the inner radius of the capillary tube and $d$ the liquid phase thickness. Although the apparent simplicity of this equation when compared to equation 13 would indicate that capillary columns are the medium of choice for the chromatographic study of diffusion phenomena, these systems suffer from the same diffi- 
culty encountered for packed columns, the poor definition of the stationary phase film thickness, which will be discussed in the following section.

\section{EXPERIMENTS AND CALCULATIONS}

IGC determinations of diffusivity in the polymer phase are made from a study of probe peak broadening at varying carrier gas velocities; a schematic illustration of typical results is shown in Figure 1. The slope at high flow rates is often identified with the quantity $C$ in the van Deemter equation (equation 2) and the diffusion coefficient can be calculated from $C$ according to equation 7. Such a calculation assumes that liquid phase mass transfer is the primary mechanism of peak spreading in this region of high carrier gas flow velocity. If the other kinetic processes discussed in the previous section are operative then the limiting slope at high flow rate of Figure 1 should also reflect these contributions as indicated by equations 8 and 14 . The contribution of each of these terms is calculated for the system octadecane-linear polyethylene (SRM 1475) studied in this laboratory as described below. The polymer was coated onto 180-150 $\mathrm{m}$ diameter (80-100 mesh) glass beads from a hot xylene solution by slowly evaporating the solvent. The coated beads were then packed into $6.35 \mathrm{~mm}$ outer diameter stainless steel mass fraction of polymer were employed. These are designated as column $A$ and $B$ respectively in the remainder of the report. About $0.1 \mathrm{\mu l}$ of 1 iquid, either neat octadecane $\left(\mathrm{C}_{18}\right)$ or a $0.5 \%$ by mass heptane solution of $\mathrm{C}_{18}$, was injected and the output of a flame ionization detector recorded on a strip chart recorder operating with a high chart speed. At least three symmetrical peak were collected for a determination of the mean width at half maximum at each carrier gas flow rate. A symmetrical peak is defined as that having a front to rear tangent slope ratio of $1 \pm 0.2$. The helium carrier gas flow rate was measured with a soap film flow meter. Inlet pressure was monitored with a calibrated pressure gauge of appropriate range while the outlet pressure was atmospheric in all cases. Concurrent injections of methane gas, assumed to have $k=0$, were employed to 
determine $t_{M}$ of equation 6 . An electronic integrator was used to time the peak maxima to within $0.01 \mathrm{~min}$. All experiments were conducted at a column temperature of $150 \pm 0.3^{\circ} \mathrm{C}$. The column capacity factor is 17.84 for $\mathrm{A}$ and 118.8 for $B$ under these conditions. A plot of $H$ vs $u$ was constructed for eight flow velocities in the high flow rate region and linear least squares fit yielded a slope for column $A$ of $7.05 \times 10^{-3}$ s with calculated standard deviation of $8.8 \%$ and $1.89 \times 10^{-2} \mathrm{~s}$ with calculated standard deviation of $7.4 \%$ for $B$.

Gas Phase Contributions

Before the gas phase contributions to the experimentally determined slopes can be estimated, a value for the diffusivity of $C_{18}$ in helium at $150^{\circ} \mathrm{C}$ is required. Two methods were used to estimate this diffusion coefficient, one attributed to Arnold (14) and a treatment of Fuller, Schettler, and Giddings (15). The following parameters were used in the calculation according to Arnold: $V_{A}=7.21 \mathrm{~cm}^{3} / \mathrm{mol}, V_{B}=407 \mathrm{~cm}^{3} / \mathrm{mol}, C_{A}=78, C_{B}=866$. The FSG treatment employed $v_{A}=2.88$ and $v_{B}=372.2$. At $150^{\circ} \mathrm{C}$ and a column outlet pressure of 1 atm the two treatments give values of 0.317 and $0.268 \mathrm{~cm}^{2} / \mathrm{s}$ respectively for the diffusion coefficient. An intermediate value of $0.292 \mathrm{~cm}^{2} / \mathrm{s}$ is used in all subsequent calculations.

llass transfer in the gas phase can be estimated from equation 9 if values of $C_{D}$ and $d_{g}$ are available. The distance over which lateral gaseous diffusion must take place depends both on the average separation between two liquid phase areas and the degree of coverage of the support surface. A probe vapor molecule diffusing to a support surface not covered by the polymer phase will continue to diffuse in the gas phase until the polymer is encountered, thus lengthening the diffusion path. The distance $d_{g}$ is assumed to be on the order of the average particle diameter. A value of 0.115 is assumed for the constant $C_{D}$ based on the theoretical limiting result discussed earlier. For averaqe size particle nf $165 \mu \mathrm{m}$, D for column $A$ is calculated as follows: 


$$
\begin{aligned}
D & =\frac{0.115(17.84)^{2}\left(1.65 \times 10^{-2} \mathrm{~cm}\right)^{2}}{\left(0.292 \mathrm{~cm}^{2} / \mathrm{s}\right)(1+17.84)^{2}} \\
& =9.61 \times 10^{-5} \mathrm{~s}
\end{aligned}
$$

A value of $1.05 \times 10^{-4} \mathrm{~s}$ is obtained for column $\mathrm{B}$. The choice of the average particle diameter for the distance $d_{g}$ was checked by calculating $D$ for the systems studied by Kieselbach (6) and comparing the calculated values to his experimental results. The agreement to within a factor of 2 in the five cases studied lends support to the choice of $d_{g}$ for the mean path length.

The $E$ term employed to account for interaction of different flow chanels of unequal velocity has been studied by Giddings (16). When parameters typical of a packed nonporous support are assumed the following approximate equation is obtained for $C_{E}$ :

$$
C_{E}=\frac{0.63 k+0.43}{k+1}
$$

Substituting this equation and data for column $A$ into equation 10 allows the $E$ term to be calculated as shown:

$$
\begin{aligned}
E & =\frac{(0.63(17.84)+0.43)\left(1.65 \times 10^{-2} \mathrm{~cm}\right)^{2}}{(17.84+1)\left(0.292 \mathrm{~cm}^{2} / \mathrm{s}\right)} \\
& =5.77 \times 10^{-4} \mathrm{~s}
\end{aligned}
$$

The corresponding value for column $B$ is $5.86 \times 10^{-4} \mathrm{~s}$.

The correlation between the $D$ and $E$ terms can be estimated from equation 11 if the correlation coefficient $\theta$ is known. Independent variables have a $\theta$ of zero while strict correspondence between two variables gives a value of unity. The value of this coefficient is not certain but estimates for capillary columns (8) suggest that 0.8 is a reasonable choice. Substituting into the equation for column A gives:

$$
\begin{aligned}
F & =\frac{2(0.8)(0.115 \times 0.619)^{1 / 2}(17.84)\left(1.65 \times 10^{-2} \mathrm{~cm}\right)^{2}}{\left(0.292 \mathrm{~cm}^{2} / \mathrm{s}\right)(1+17.84)} \\
& =3.77 \times 10^{-4} \mathrm{~s}
\end{aligned}
$$


The corresponding result for column $B$ is $3.98 \times 10^{-4} \mathrm{~s}$.

The last gas phase contribution to peak spreading that will be considered arises from column coiling, a component of the $\mathrm{H}_{\text {tc }}$ term of equation 13. The contribution of this term can be estimated from an equation given for columns of circular tube cross section (10) as:

$$
H_{t c}=\frac{7 r_{0}^{4} \tau^{2}}{12 R_{0}^{2} D_{g}}
$$

where $r_{0}$ is the tube radius, $R_{0}$ the coil radius, and $\tau$ the tortuosity, which should have a value of 0.63 for packed, impenetrable spheres (17). The columns employed in this study have an inner radius of $0.229 \mathrm{~cm}$ and a coil radius of $5.72 \mathrm{~cm}$ leading to an $\mathrm{H}_{\text {tc }}$ term of $6.62 \times 10^{-5} \mathrm{~s}$.

The gas phase contributions to peak spreading are summarized in the following table and compared to the limiting slope C calculated from the van Deemter equation least squares fit.

Table 2 Gas Phase Contributions to Peak Spreading

\begin{tabular}{|c|c|c|c|c|c|c|}
\hline Column & $D(\mathrm{~ms})$ & $E$ & $\mathrm{~F}$ & $\mathrm{H}_{t c}$ & $\Sigma$ gas phase & C \\
\hline A & 0.096 & 0.577 & 0.377 & 0.066 & 1.12 & 7.05 \\
\hline B & 0.105 & 0.586 & 0.398 & 0.066 & 1.16 & 18.9 \\
\hline
\end{tabular}

It is evident that the peak spreading attributed to gas phase diffusion can make a significant contribution to the overall kinetic broadening and plate height. Equations that neglect these contributions should be applied only when they are assuredly negligible. Gas phase diffusion contributions can be minimized by ellploying low molecular weight carrier gases at high temperatures to increase the diffusivity $\mathrm{D}_{\mathrm{g}}$.

The capacity factor also contributes to the magnitude of the $C, D$, and $F$ terms. As the magnitude of $k$ increases, the $k^{2} /(1+k)^{2}$ term of $D$ (equation 9) approaches unity while the $k /(1+k)^{2}$ term of $C$ approaches zero as illustrated in Table 3. 
Table 3 Magnitude of Various k-dependent Terms

$\begin{array}{rlllc}k & \frac{k}{(1+k)^{2}} & \frac{k^{2}}{(1+k)^{2}} & \frac{k}{1+k} & \frac{1+6 k+11 k^{2}}{24(1+k)^{2}} \\ 1 & .25 & .25 & .5 & .118 \\ 10 & .083 & .83 & .909 & .399 \\ 100 & .0098 & .98 & .990 & .452 \\ 1000 & .0010 & .998 & .999 & .458\end{array}$

A large capacity factor may cause the gas phase mass transfer terms to greatly exceed the stationary phase term; an example is provided in a study by Kieselbach (6). His findings indicate that the $C$ term is dominant for early sample peaks (k 2 ) but becomes insignificant for late peaks (k 30$)$ primarily due to the influence of the $k /(1+k)^{2}$ factor as indicated in Table 3 . The gas diffusion terms are thought to be large when $k$ is not near unity and the stationary phase loadings are less than a mass fraction of 0.1 . A determination of the probe-stationary phase diffusion coefficient is favored when the probe has a $k$ value near unity since the $C$ term should be prominent. Increasing the stationary phase loading in order to maximize the $C$ term by thickening the coating can be partially offset by the expected increase in retention time and corresponding decrease in $k /(1+k)^{2}$. Polymer Phase Contribution

The stationary phase contribution to peak broadening is customarily related by the van Deemter $\mathrm{C}$ term, found from a plot of $\mathrm{H}$ vs. $\mathrm{u}$ at large $\mathrm{u}$, to the diffusivity as indicated in equation 7 . Such a procedure can only succeed if the other contributions to peak spreading discussed previously are small when compared to that arising from liquid or stationary phase mass transfer. This has been assumed to be the case in many previous studies of probe-polymer diffusion. A further consideration is the nature of the carrier velocity on which the plate height depends. Equation 14 indicates that while the plate height contribution of the $B, D, E$, and $F$ terms are directiy or inversely proportional to the carrier 
outlet velocity $u_{0}$, the $C$ term contribution to $\bar{H}$ depends on the average velocity, in this case a distance average. Consequently, the often-employed plot of $\mathrm{H}$ vs. carrier velocity suggested by equation 2 will not be linear at high flow velocity for any variant of velocity if both liquid and gas phase mass transfer effects are nonnegligble. Purnell (9) has suggested a method for resolving the liquid and gas phase mass transfer contributions which involves first determining $A$ and $B$ of equation 14 and employing these values to reduce the experimental $\bar{H}$ to the peak spreading arising only from mass transfer effects, $H_{M T}$. In terms of an equation:

$$
H_{M T} / u_{0}=\left(\bar{H}-A-\frac{B}{u_{0}}\right) / u_{0}=\frac{2 p_{0}}{p_{i}+p_{0}} C+D+E+F
$$

When the left-hand side of this equation is plotted against the pressure correction $2 p_{0} /\left(p_{i}+p_{0}\right)$ a straight line with slope $C$ and intercept $(D+E+F)$ should be obtained. Evaluation of the constants $A$ and $B$ requires that experiments be conducted over a wide range of carrier gas flow velocities. Limited low velocity data precludes experimental determination of these constants in many cases; however, they can be estimated from theoretical considerations. The multipath A term defined in equation 3 has been found to have a $\lambda$ value between one half and unity in many studies (18) and a value of 0.5 is assumed for these calculations. The longitudinal diffusion $B$ term most often has a $\gamma$ value near one (18) and unity is assumed here. These values were used to calculate the $A$ and $B$ terms of equation 18 and a fit of $H_{M T} / u_{0}$ against the pressure correction term gives a linear least squares 1 ine with a slope of $1.64 \times 10^{-2} \mathrm{~s}$ and calculated standard deviation of $5.5 \%$ for column $\mathrm{A}$ and $2.19 \times 10^{-2} \mathrm{~s}$ with calculated standard deviation of $6.6 \%$ for $B$. The smaller standard deviations of these slopes compared to the corresponding values for the van Deemter fit discussed earlier indicate the better applicability of the Purnell method. The $C$ value obtained by this method for Column $A$ is more than twice that obtained from the simple van Deemter analysis. This is attributed to the latter's combination of the effects of polymer and gas phase mass transfer in a nonlinear manner 
which can be noted by comparing the $C$ term obtained from equation 2 to its complement in equation 14, the expression in parenthesis. For the case of column $A$ where the gas phase terms contribute significantly to the peak broadening, the van Deemter equation based analysis underestimates the magnitude of $C$. Gas phase mass transfer effects for column B contribute only $6 \%$ to the total broadening, as indicated in Table 2, and this is reflected in the similar results of the two methods for determining $\mathrm{C}$ which differ by onty $15 \%$.

While the method of determining the polymer phase mass transfer contribution can be important under certain column conditions, the most significant parameter affecting a diffusivity determination is the distance over which diffusion occurs. This distance is identified with the effective film thickness $d_{f}$ of equation 7. It is often assumed that the stationary phase is distributed as a uniform thin film over the surface of the spherical glass bead column packing. The effective film thickness for this geometry can be calculated from the expression:

$$
d_{f}=\frac{M_{2}}{4 \pi r^{-2} N \rho}
$$

where $M_{2}$ is the mass of polymer phase in the column, $\bar{r}$ the average support particle radius, $\mathrm{N}$ the number of support particles in the column, and $\rho$ the polymer density. The results are $3.46 \times 10^{-6} \mathrm{~cm}$ for $A$ and $3.24 \times 10^{-5} \mathrm{~cm}$ for column $B$. Such $a$ configuration requires a high amount of stationary phase surface area, however, and a geometry in which the 1 iquid phase is confined by capillary forces into annular regions around the contact points between support particle spheres has been advocated as a means of minimizing the exposed stationary phase area. This geometry has been observed for both low molecular weight (11) and polymeric (19) stationary phases coated onto glass bead supports. A factor of three decrease in the surface to volume ratio of polystyrene coatings in IGC columns on prolonged treatment at $180^{\circ} \mathrm{C}$ led Klein and Waltnitzki (20) to suggest that surface tension can be effective in minimizing the surface area of the supported polymer at elevated temperatures. 
Giddings (21) has compared the contribution to peak spreading expected from a hypothetical support coated with two regions, a thin film of uniformly adsorbed stationary phase and a second region where the liquid phase collects at the support contact points, and concludes that for glass bead supports at mass fraction loadings up to about 0.015 the bulk of the liquid phase is present at the bead contact points and controls the plate height performance by virtue of its greater thickness. An equation has been proposed $(22)$ to relate $C$ to measurable column parameters for this geometry and is as follows:

$$
C=\frac{d_{p}{ }^{2} k}{12 D_{\ell}(1+k)^{2}}\left(\frac{m_{2} \rho_{s}}{18.75 \rho}\right)^{1 / 2}
$$

where $\rho_{s}$ is the density of the support particles and $m_{2}$ the mass fraction of polymer phase in the column. This treatment assumes that the spherical support particles pack randomly and are surrounded by 6.25 nearest neighbors. The relation for $C$ is similar to equation 7 differing only by the explicit expression for $d_{f}$, given in terms of the particle diameter as:

$$
d_{f}=\left(\frac{m_{2} \rho s}{18.75 \rho}\right)^{1 / 4} \quad \frac{d_{p}}{4 \sqrt{5}}
$$

The average film thickness calculated for a polymer stationary phase collected at bead contact points is $5.28 \times 10^{-4} \mathrm{~cm}$ for column $A$ and $9.24 \times 10^{-4} \mathrm{~cm}$ for column $B$.

Both equations 7 and 20 have been used to calculate the diffusion coefficient of octadecane in HDPE. For column $A$ assuming a thin uniform film, $D_{\ell}$ can be calculated by substituting equation 19 into equation 7 :

$$
\begin{aligned}
D_{\ell} & =\frac{2}{3} \frac{17.84}{(1+17.84)^{2}\left(1.64 \times 10^{-2} \mathrm{~s}\right)}\left(\frac{6.10 \times 10^{-4} \mathrm{~g}}{4 \pi\left(8.25 \times 10^{-3} \mathrm{~cm}\right)^{2} \times 2.11 \times 10^{5} \times 0.978 \mathrm{~g} / \mathrm{cm}^{3}}\right)^{2} \\
& =2.4 \times 10^{-11} \mathrm{~cm}^{2} / \mathrm{s}
\end{aligned}
$$

Assuming a geometry where the polymer collects at the bead contact points for column $A, D_{\ell}$ is calculated according to equation 20 as follows: 


$$
\begin{aligned}
D_{\ell} & =\frac{\left(1.65 \times 10^{-2} \mathrm{~cm}\right)^{2}(17.84)}{12\left(1.64 \times 10^{-2} \mathrm{~s}\right)(1+17.84)^{2}}\left(\frac{6.10 \times 10^{-4} \mathrm{~g} \times 2.019 \mathrm{~g} / \mathrm{cm}^{3}}{18.75 \times 0.978 \mathrm{~g} / \mathrm{cm}^{3}}\right)^{1 / 2} \\
& =5.7 \times 10^{-7} \mathrm{~cm}^{2} / \mathrm{s}
\end{aligned}
$$

The corresponding values for column $B$ are $2.6 \times 10^{-10}$ and $2.1 \times 10^{-7} \mathrm{~cm}^{2} / \mathrm{s} \mathrm{re-}$ spectively. It is evident that the uniform thin film geometry assumption leads to unreasonably low values of the diffusion coefficient. In addition, an order of magnitude discrepancy exists between results for the two columns. The assumption of a collection of polymer stationary phase at bead contact points yields diffusion coefficients of reasonable magnitude and column-to-column agreement within a factor of three. Limited data are available in the literature on diffusion of the higher alkanes in HDPE; however, studies of similar diffusants, linear aliphatic esters with 25 to 45 backbone units, have been conducted by Klein and Briscoe (23). Extrapolating their experimental results to an 18 unit chain leads to an expected diffusion coefficient of $5 \times 10^{-6} \mathrm{~cm}^{2} / \mathrm{s}$ in linear polyethylene at $152^{\circ} \mathrm{C}$. While the two results cannot be compared directly due to the different chemical nature of the diffusion species and the chain length extrapolation required, their agreement within an order of magnitude is encouraging.

An IGC study of diffusion of benzene and carbon tetrachloride in polyethylene containing columns has shown that the diffusion coefficient increases by a factor of four to nine as the stationary phase film thickness is increased to four times the original value (24). These authors propose that the short contact time between probe vapor and polymer results in only a portion of the film, termed the effective layer, taking part in probe exchange between the polymer and vapor phase. The thickness of the effective layer is thought to increase more slowly than the overall increase in film thickness. The existence of an effective layer could further complicate the film geometry question since the geometrically determined thickness would not correspond to the distance over which diffusion takes place. The diffusion coefficient determined in 
this study decreased by a factor of three as the thickness of the film collected at the support contact points approximately doubled. This trend is contrary to the benzene and carbon tetrachloride results previously discussed. The effective layer concept does not apply to these polyethylene-octadecane studies since the contact time between polymer and probe is sufficiently long that diffusion of at least two thirds of the probe molecules through the entire film thickness is possible for each "theoretical plate" of column length.

The distribution of film thickness also plays an important role in determining the magitude of the diffusivity. The previous calculations assume that the film thickness is constant. In an actual column, however, the film thickness would be expected to be distributed about the mean values employed here. An alternate form of equation 7 can be used to estimate the effects of different film thicknesses. The equation is:

$$
C=\frac{k}{D_{\ell}(1+k)^{2}} \sum_{i} q_{i} x_{i} d_{i}^{2}
$$

where $q_{i}$ is a configuration factor and $x_{j}$ the volume fraction per unit column volume of stationary phase segments with thickness $d_{i}(25)$. Assuming a uniform configuation factor and holding $x_{i}$ constant gives:

$$
c=\frac{k q x}{D_{l}(1+k)^{2}} \sum_{i} d_{i}^{2}=z \sum_{i} d_{i}^{2}
$$

which reduces to:

$$
C=z d^{2}
$$

for a uniform film of thickness $d$. For a distribution with an equal volume of film $0.1 d$ and 10d in thickness:

$$
C^{\prime}=z \sum 5\left(\frac{d}{10}\right)^{2}+\frac{1}{20}(10 d)^{2}=5.05 d^{2}
$$

This distribution, resulting from the same volume of stationary phase present in the uniform thickness case, leads to a liquid mass transfer coefficient five 
times larger and a diffusivity value five times smaller than those corresponding to a uniformly distributed film. Table 4 summarizes the effects of different thickness distributions on the diffusion coefficient $D_{l}^{\prime}$ compared to that expected from a uniform film of thickness $d, D_{\ell}$. All the geometries considered require the same volume of stationary phase per unit column volume and have an average thickness of $d$.

Table 4 Effect of Thickness Distribution on Diffusivity in the Polymer Phase

$\begin{array}{cccc}\begin{array}{c}\text { Thin Section } \\ \text { Depth }\end{array} & \begin{array}{c}\text { Thick Section } \\ \text { Depth }\end{array} & C^{\prime} / C & D_{\ell}^{\prime} / D_{\ell} \\ \text { d/2 } & 2 d & 1.25 & 0.80 \\ d / 4 & 4 d & 2.13 & 0.47 \\ d / 10 & 10 d & 5.05 & 0.20 \\ d / 50 & 50 d & 25.01 & 0.04 \\ d / 100 & 100 d & 50.01 & 0.02\end{array}$

It is evident from these examples that thick sections of the polymer film can exert a disproportionate influence and lead to a considerable reduction in the polymer-probe diffusion coefficient when compared to the value calculated for an IGC experiment that assumes an ideal polymer layer with uniform thickness.

Concentration Effects

The diffusivity is often quite sensitive to the concentration of the diffusing species, depending approximately either linearly or exponentially on concentration (26). In the IGC determination of the diffusion coefficient discussed previously, the probe concentration in the nolymer filn varies both along the column length due to increasing peak broadening with transit time and with the depth of penetration into the polymer film expected from Fickean diffusion. The diffusivity determined is therefore a concentration-averaged value over all column conditions. The actual concentration of octadecane in the polymer film 
has been estimated to range from 0.01 to 1.5 weight percent for column $A$ and from 0.001 to 0.16 for column B. The diffusion coefficients calculated for column B correspond to concentrations about an order of magnitude lower than for column A.

\section{Capillary columns}

The plate height equation for capillary columns (equation 15) is superior to that for packed columns by virtue of its analytic expression for the cas phase mass transfer terms. Experiments with low capacity factor probes favor operation in a region where liquid mass transfer effects are prominent, as indicated by Table 3, a situation similar to that for packed columns. Equation 15 was derived for a uniform thin coating of stationary phase on the inner tube wall. In actual practice the stationary phase coating will never be uniform and the film geometry is envisioned as similar to the case of a porous diatomite support where most of the stationary phase is contained in surface pores of varying dimensions (11). The geometry of the polymer film on the capillary wall is thought to be even more irregular than that of a film collected at the contact points of a spherical glass bead support. An ill-defined diffusion distance, a consequence of the uncertain film geometry, leads to similar difficulties in calculating the polymer-probe diffusivity from the liquid mass transfer term as discussed above for packed columns.

\section{Adsorption effects}

This discussion has neglected any effect of interfacial adsorption on peak spreading but such a mechanism may be operable at the polymer-support interface and even override the liquid phase mass transfer induced broadening in some cases if the sorption isotherm is nonlinear. Several expressions for adsorption-desorption kinetic contributions are available (11). The nonpolar nature of the system examined in these studies and the symetry of the resulting chromatographic elution peaks suggest that adsorption contributions to peak spreading are minimal. 
OTHER IGC STUDIES OF DIFFUSION IN POLYMERS

Several attempts have been made to compare experimental $C$ terms to those calculated from equation 20 for the low molecular weight stationary phase trio-tolyl phosphate on glass bead supports. Such a comparison can serve as an indication of the maximum possible accuracy to which the stationary phase-probe diffusion coefficient can be determined. The two quantities agree within about $30 \%$ for loadings between 0.1 and $0.25 \%$ by mass on 540 and $980 \mu \mathrm{m}$ diameter beads with the calculated value becoming too small by a factor of two as the loading reached $1 \%$ by mass (22). A more detailed study (27) revealed that the calculated and experimental C agreed to about 10\% for $230 \mu \mathrm{m}$ diameter beads whereas for smaller beads (95 $\mu \mathrm{m}$ diameter) the two terms concur within a factor of 1.5. Column loadings ranged between 0.09 and 1.05\%. Agreement is poor when smaller support diameters $(40 \mu \mathrm{m})$ or liquid loadings greater than $1 \%$ are examined, the calculated value being at least a factor of three smaller than the experimental. These data suggest that a minimum uncertainty in $D_{l}$ of about $10 \%$ is 1 ikely even under the most favorably conditions.

A number of IGC studies have been concerned with diffusion in silicone elastomers (28-31). Each of these involve determinations with the polydimethylsiloxane SE-30; however, data on diffusion in the oligomers of this material (28-31), polysiloxanes with aromatic sidegroups (30), and polyethylene glycol are also reported. All the SE-30 studies assume that the polymer is held solely at the supporting bead contact points. Experimental problems with sample injection process and neglect of gas phase mass transfer effects complicate the interpretation of results presented in the early studies (28-30). These problems have been overcome in the work of Millen and Hawkes (31) who studied diffusion of alkanes in SE-30 at temperatures from 50 to $200^{\circ} \mathrm{C}$. Diffusion coefficients in the range of $10^{-7}$ to $10^{-5} \mathrm{~cm}^{2} / \mathrm{s}$ are reported for the alkanes undecane to octadecane and Arrhenius activation energies of 25 to $60 \mathrm{~kJ} / \mathrm{mol}$ can be calculated from their data. 
Diffusion of decane and tetradecane in LDPE has been examined in the $1 \mathrm{GC}$ studies of Gray and Guillet (32). They base their calculations on a uniform polymer film present on the surface of the glass bead support. They found diffusivities for tetradecane ranging from 1 to $7 \times 10^{-8} \mathrm{~cm}^{2} / \mathrm{s}$ over the temperature interval from 125 to $170^{\circ} \mathrm{C}$ while a decane diffusant gave values of 0.4 to 1.3 $\times 10^{-8} \mathrm{~cm}^{2} / \mathrm{s}$ from 30 to $80^{\circ} \mathrm{C}$. The LDPE-tetradecane results obtained above the polymer melting point follow an Arrhenius type temperature dependence and an activation energy of $67 \mathrm{~kJ} / \mathrm{mol}$ can be calculated from their data. The authors attribute a changing degree of polymer crystallinity with temperature for the failure of the LDPE-decane results to reflect an Arrhenius temperature dependence. If the polymer is assumed to reside primarily at the bead contact points rather than being uniformly distributed on the support surface, equation 20 can be used to calculate the diffusion coefficient from the tabulated $C$ data of reference 32. For the mean support particle diameter of $340 \mu \mathrm{m}$, the diffusivity in LDPE is calculated to be 5.1 to $44 \times 10^{-6} \mathrm{~cm}^{2} / \mathrm{s}$ for tetradecane and 2.1 to $8.1 \times 10^{-6} \mathrm{~cm}^{2} / \mathrm{s}$ for decane over the same temperature ranges indicated previous $7 y$. The desorption and time lag experiments on decane diffusion in LDPE by McCall and Slichter (33) yield a diffusivity of $7.5 \times 10^{-9} \mathrm{~cm}^{2} / \mathrm{s}$, in better agreement with the result of Gray and Guillet based on a uniform film assumption.

Further studies on diffusion in LDPE above the melting point are reported by Braun, Poos, and Guillet (34). Results are given for dodecane, tetralin, two isomers of decalin, BHA, and BHT and are on the order of $10^{-7} \mathrm{~cm}^{2} / \mathrm{s}$ in all cases. Activation energies are also given and range from 38 to $42 \mathrm{~kJ} / \mathrm{mol}$. A uniformly distributed polymer film was again assumed. These diffusion data were criticized by Millen and Hawkes (35) who maintained that equation 5 was employed incorrectly for the calculation. They present revised results obtained by recalculating with equation 7 and indicate that the initial work's neglect of gas phase mass transfer effects could introduce an error of about $5 \%$ in the recalculated diffusivity values. 
Diffusion of toluene and decane in polystyrene has been studied by Gal in and Rupprecht (36) for temperatures of 160 to $180^{\circ} \mathrm{C}$. They determined diffusivities by IGC of about $3 \times 10^{-7} \mathrm{~cm}^{2} / \mathrm{s}$ from the van Deemter equation, determining the film thickness from the surface to volume ratio by studying the surface adsorption of decane below the polymer $\mathrm{Tg}$.

Three amorphous polymers have been examined at temperatures above and below their glass transition by Tait and Abushihada (37). They studied diffusion of buytl ether, ethyl benzene, and propyl benzene in PVC, PS, and PMMA at 45 and $110^{\circ} \mathrm{C}$. The polymer was assumed to be uniformly distributed on the Chrom-W and glass bead supports empioyed. Diffusion coefficients on the order of 1 to $2 \times 10^{-8} \mathrm{~cm}^{2} / \mathrm{s}$ were determined in all cases. Very little change in the diffusivity was noted for a given polymer-solvent pair when the measurement temperature shifted from below to above the polymer glass transition, a finding contrary to the expected increase in diffusivity by several orders of magnitude when passing from a glassy, solid polymer below $\mathrm{Tg}$ to a viscous liquid with greatly increased free volume above $\mathrm{Tg}$.

CONCLUSIONS AND FUTURE WORK

The previous discussion has shown that gas phase mass transfer effects can be estimated and their interference with IGC studies of probe-polymer diffusion minimized by the choice of appropriate column conditions. A more difficult problem arises in accurately defining the mean polymer phase thickness and thickness distribution. These parameters have a strong influence on the magnitude of the diffusivity, yet there is considerable uncertainty associated with them as discussed above. For these reasons the probe-polymer diffusion coefficient probably cannot be determined any more accurately than within a factor of ten even under the most favorable conditions.

While accurate absolute diffusivity measurements seem beyond the reach of the IGC method, the possibility exists for using the technique to rank the relative 
ease of diffusion of many probes in a given polymer. A calibration of a specific column can be accomplished by conducting experiments with a probe-polymer combination of known diffusivity to calculate the mean polymer layer thickness from the measured peak broadening. The same column can then be used under similar operating conditions to determine other probe diffusivities and a relative ranking of many probe diffusion coefficients established. Such experiments are now in progress.

The diffusivity determinations described in this report have been conducted above the polymer glass transition temperature. Studies below the polymer $\mathrm{Tg}$ are also being attempted. These experiments are complicated by a competition between surface adsorption and bulk sorption as the primary probe retention mechanism. In addition, the slow nature of diffusion in glass-like materials may prohibit the entire polymer layer from taking part in probe sorption and only an "effective layer" may participate, again complicating the film thickness issue. 
1. W. E. Hammers and C. L. DeLigny, J. Polym. Sci., Phys., 12, 2065 (1974).

2. Y.-K. Leung and B. E. Eichinger, J. Phys. Chem., 78, 60 (1974).

3. J. J. van Deemter, F. J. Zuiderweg and A. Klinkenberg, Chem. Eng. Sci., $\underline{5}, 271(1956)$.

4. J. Bohemen and J. H. Purne11, "International Gas Chromatography Symposium at Amsterdam, 1958", D. H. Desty, ed., Butterworths, London, 1958, p. 6.

5. J. C. Giddings, Nature, 188,847 (1960).

6. R. Kieselbach, Ana1. Chem., 33, 23 (1961).

7. J. C. Giddings, S. L. Seager, L. R. Stucki, and G. H. Stewart, Anal. Chem., 32, 867 (1960).

8. W. L. Jones, Ana 1. Chem., 33, 829 (1961).

9. H. Purne11, "Gas Chromatography", Wiley, New York, 1962, Ch. 8.

10. J. C. Giddings, "Dynamics of Chromatography", Marcel Dekker, New York, 1965, Ch. 2.

11. J. C. Giddings, Anal. Chem., 35, 439 (1963).

12. S. Dal Nogare and R. S. Juvet, "Gas-Liquid Chromatography", Interscience, New York, 1962, Ch. 5.

13. M. J. E. Golay, "International Gas Chromatography Symposium at Amsterdam, 1958", D. H. Desty, ed., Butterworths, London, 1958, p. 36.

14. J. H. Arnold, Ind. Eng. Chem., 22, 1091 (1930).

15. E. N. Fuller and J. C. Giddings, J. of Gas Chromatogr., 3, 222 (1965).

16. J. C. Giddings, Ana1. Chem., 34, 1186 (1962).

17. J. C. Giddings, "Dynamics of Chromatography", Marcel Dekker, New York, 1965, p. 246.

18. H. Purne11, "Gas Chromatography", Wiley, New York, 1962, Ch. 9.

19. G. J. Courval and D. G. Gray, Can. J. Chem., 54, 3496 (1976).

20. J. Klein and G. Maltnitzki, Polym. Bu11. Berlin, 1, 451 (1979). 
21. J. C. Giddings, Ana1. Chem., 34, 458 (1962).

22. J. C. Giddings, K. L. Mallik and M. Eikelberger, Anal. Chem., 34, 1026 (1962).

23. J. Klein and B. J. Briscoe, Proc. R. Soc. Lond. A., $\underline{365}, 53$ (1979).

24. Ye. N. Rosolovsaya and Ya. Sal'vinski, Polym. Sci. USSR, 18, 1638 (1976).

25. J. C. Giddings, "Dynamics of Chromatography", Marcel Dekker, New York, 1965 , p. 141.

26. J. Crank and G. S. Park, "Diffusion in Polymers", Academic Press, London, 1968, p. 17.

27. S. J. Hawkes, C. P. Russe11, and J. C. Giddings, Anal. Chem., 37, 1523 (1965).

28. S. J. Hawkes, and D. J. Carpenter, Anal. Chem., 39, 393 (1967).

29. L. Butler and S. Hawkes, J. Chromatogr. Sci., 10, 518 (1972).

30. J. M. Kong and S. J. Hawkes, J. Chromatogr. Sci., 14, 279 (1976).

31. W. Millen and S. Hawkes, J. Chromatogr. Sci., 15, 148 (1977).

32. D. G. Gray and J. E. Guillet, Macromolecules, 6, 223 (1973).

33. D. W. McCall and W. P. Slichter, J. Amer. Chem. Soc., 80, 1861 (1958).

34. J.-M. Braun, S. Poos, and J. E. Guillet, J. Polym. Sci., Letters, 14, 257 (1976).

35. W. Millen and S. J. Hawkes, J. Polym. Sci., Letters, 15, 463 (1977).

36. M. Galin and M. C. Rupprecht, Polymer, 19, 506 (1978).

37. P. J. T. Tait and A. M. Abushihada, J. Chromatogr. Sci., 17, 219 (1979). 


\section{SECTION $V$}

Fluorescence Measurements of Antioxidant Migration from Plastics 


\section{Introduction}

Since the fluorescence technique is selective and sensitive and does not require inordinately expensive equipments, it is suitable for the study of the migration from plastics of antioxidants that are often strongly fluorescent. We have developed a procedure to measure by fluorescence technique such migration and have applied the procedure to determine the migration of $\mathrm{N}, \mathrm{N}^{\prime}$-diphenyl-p-phenylenediamine from low-density polyethylene films immersed in $n$-heptane. We describe here the procedure we have developed and the preliminary results of its application.

\section{Experimental}

Antioxidant - N,N'-diphenyl-p-phenylenediamine, hereafter referred to as DPPD, was purified by recrystallization from toluene according to the following procedures. A mixture consisting of 20.0 grams of DPPD and $950 \mathrm{cc}$ of toluene was slowly heated with stirring in a nitrogen-filled glove-bag until the temperature of the mixture reached $43^{\circ} \mathrm{C}$, whereupon DPPD solids seemed completely dissolved. The solution thus obtained was filtered by gravity with a Whatman number-42 filter paper in the glove-bag. After 5 hours during which the filtrate thus obtained cooled down slowly to $22^{\circ} \mathrm{C}$, some DPPD crystals formed. The filtrate with the crystals was placed overnight in a refrigerator at $0.5^{\circ} \mathrm{C}$. The crystals were collected on a Buchner funnel, washed with tolune chilled to $0.5^{\circ} \mathrm{C}$, and dried in a dessicator at $22^{\circ} \mathrm{C}$ and ca $10^{-2} \mathrm{~mm} \mathrm{Hg}$. 
Polyethylene - National Bureau of Standards Standard Reference Material (NBSSRM) 1476, a branched polyethylene whole polymer containing $50 \mathrm{ppm}$ of the antioxidant Santonox (Monsanto) which is 4, 4'-thio-bis-(6-t-Butyl-3-cresol) and soluble in toluene, was purified as follows. To a 2-1iter, one-neck, round-bottom flask equipped with a magnetic stirrer were added 100 grams of NBS-SRM 1476 and 2 liters of toluene. A slow stream of purified nitrogen was bubbled in the mixture for about 30 minutes and then was admitted to the flask through a ground joint loosely stoppering the flask. The contents of the flask were stirred and slowly heated with an oil bath until the bath temperature reached $110^{\circ} \mathrm{C}$, whereupon NBS-SRM 1476 seemed completely dissolved. At this point, the heating was stopped and the solution was allowed to cool down with stirring. When the bath temperature was $66^{\circ} \mathrm{C}$, the solution turned turbid. After the slurry thus obtained cooled down to $22^{\circ} \mathrm{C}$, polyethylene solids were collected on a Büchner funnel, washed with 2 liters of toluene, and dried in a dessicator at $22^{\circ} \mathrm{C}$ and ca $10^{-2} \mathrm{~mm} \mathrm{Hg}$.

Solvent - Commercial "Distilled in Glass", n-Heptane was used for fluorescence measurements and for extraction without further purification.

Blending of DPPD and Polyethylene - To a 500-ml, one-neck, round-bottom flask equipped with a magnetic stirrer were added $300 \mathrm{ml}$ of Toluene "Distilled in Glass", and 30.0 grams of polyethylene. The flask was loosely capped with a ground joint through which a slow stream of purified nitrogen flowed. Then, the contents of the flask were stirred and slowly heated with an oil bath until the bath temperature reached $105^{\circ} \mathrm{C}$, whereupon polyethylene completely dissolved and the heating was stopped, while the stirring was maintained. When the bath temperature was $100^{\circ} \mathrm{C}, 0.030$ gram of DPPD in $10 \mathrm{ml}$ of toluene was added to the polyethylene solution. The solution which turned turbid at $66^{\circ} \mathrm{C}$ was allowed to $\operatorname{cool}$ down to $51^{\circ} \mathrm{C}$, whereupon the flask was stoppered with a teflon-lined stopper and stored overnight in a refrigerator at $0.5^{\circ} \mathrm{C}$. The 
slurry thus obtained was stripped of solvent at reduced pressure with the use of a rotary evaporator, the heating being provided by a water bath at $20^{\circ} \mathrm{C}$. The DPPD-polyethylene powder thus obtained was then dried in a dessicator at $22^{\circ} \mathrm{C}$ and ca $10^{-2} \mathrm{~mm} \mathrm{Hg}$.

Fusion of DPPD-Polyethylene Powder - DPPD-polyethylene powder was fused to form a void-free mass with the use of a two-part tubular device shown in Figure 1. The lower end of the tube was 1 ined with a portion cut from a $22 \mathrm{~mm} x$ $80 \mathrm{~mm}$ soxhlet extraction thimble made from TFE (Teflon/Halon). A quantity of DPPD-polyethylene powder was pulverized in a glass mortar and 13.1 grams of it was placed in the TFE-lined portion of the tube with the use of a long-stem powder funnel, care being taken not to let the powder touch other part of the tube. The powder was then pressed into a compact mass with the use of a flatended glass rod. The two parts of the device were then joined by sealing and then the device was connected to a vacuum line equipped with a diffusion pump. The device was kept at $22^{\circ} \mathrm{C}$ and $\mathrm{ca} 10^{-7} \mathrm{~mm} \mathrm{Hg}$ for about 16 hours and then immersed in an oil bath at $95^{\circ} \mathrm{C}$. The temperature of the $0 i 1$ bath was raised to $178^{\circ} \mathrm{C}$ in two hours, whereupon the valve between the device and the vacuum line was closed. Two days later, the temperature of the oil bath was reduced from $178^{\circ} \mathrm{C}$ to $95^{\circ} \mathrm{C}$ in 90 minutes, whereupon the melt in the device became frozen and the heating of the oil bath was terminated. When the temperature of the oil bath dropped to $22^{\circ} \mathrm{C}$, the aforementioned valve was opened to the vacuum line and then the device was sealed at the constriction. 


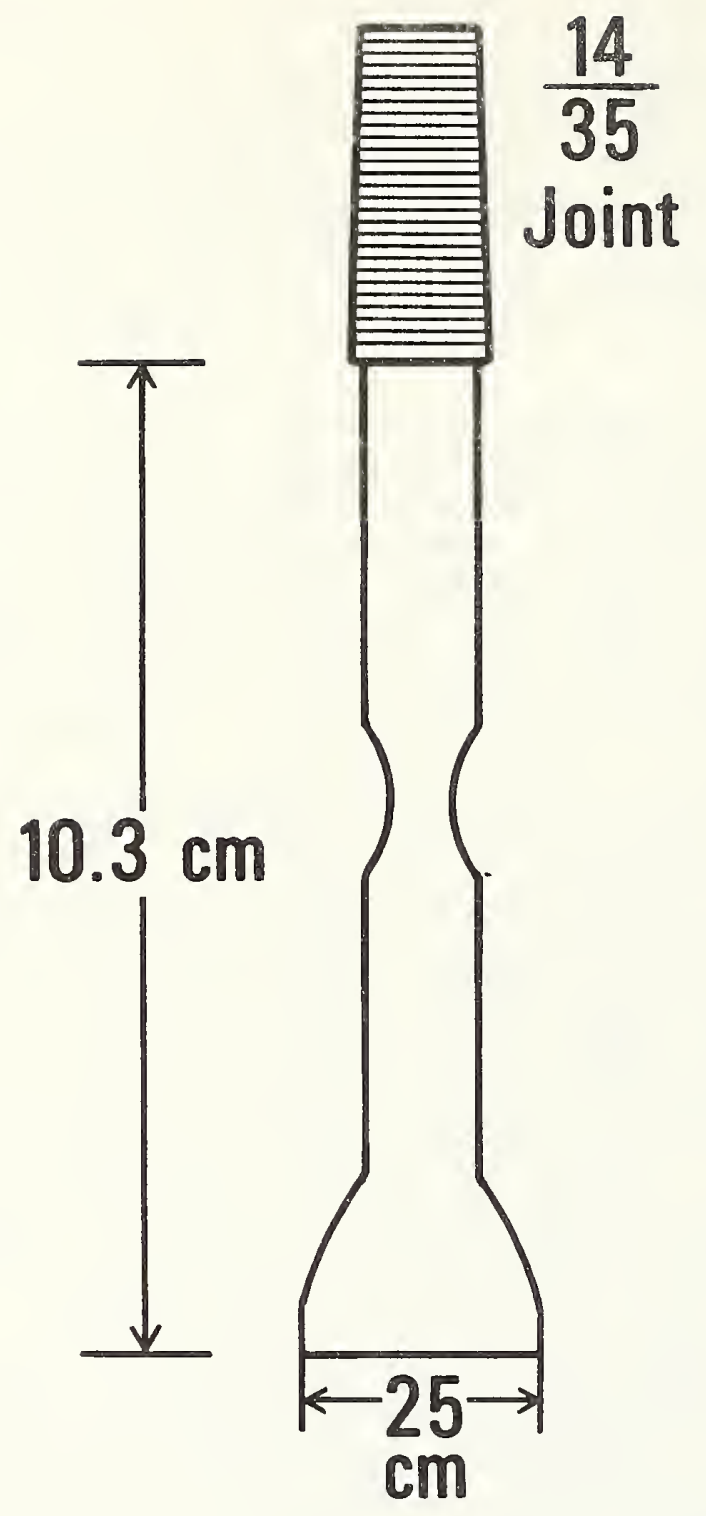

Figure 1.
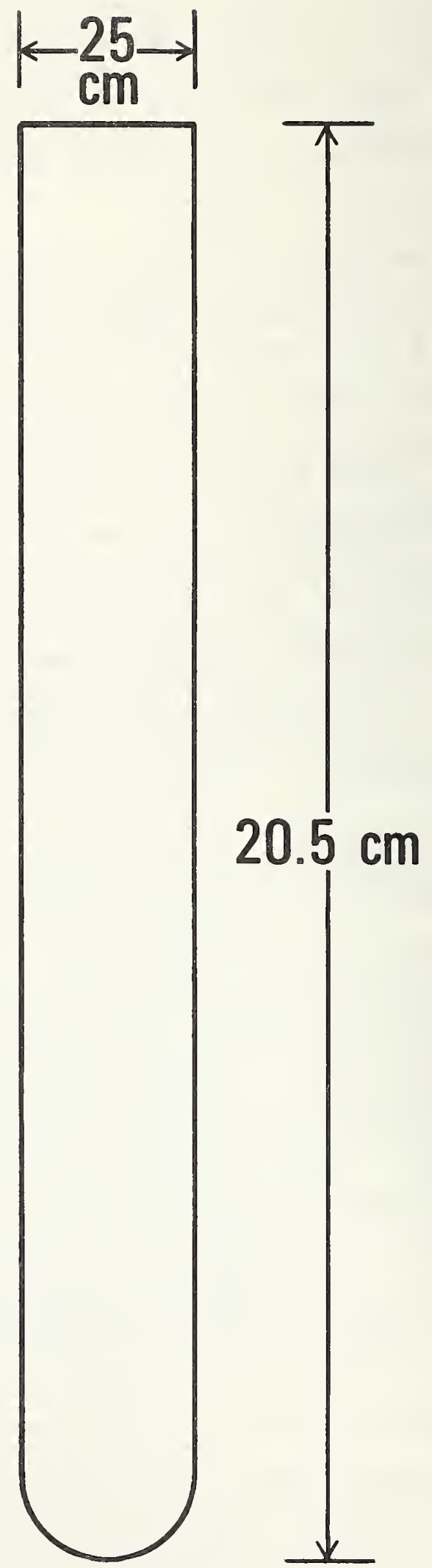

$\Rightarrow x$ 
DPPD-Polyethylene Films - The void-free DPPD-polyethylene mass was shaped into a cylinder with the use of a lathe. Immediately before an extraction experiment, films were sectioned from the DPPD-polyethylene cylinder with the use of a microtome whose knife, as well as the DPPD-polyethylene cylinder, was chilled to $1{ }^{\circ} \mathrm{C}$ just before sectioning. The average thickness, diameter, and density of the seven films thus obtained were $2.67 \times 10^{-2} \mathrm{~cm}, 1.60 \mathrm{~cm}$, and $0.91 \mathrm{~g} / \mathrm{cm}^{3}$, respectively.

Thermal Analysis - The thermal property of DPPD was determined with the use of a differential scanning calorimeter (Perkin-Elmer, DSC-2)*.

Fluorimetry - With the exception of the spectra shown in Figure 3 which were obtained with a Fluorolog Spectrofluorimeter (SPEX Industries, Inc.), a11 fluorescence measurements were obtained with an Amino-Bowman ${ }^{*}$ Spectrofluorimeter. An Analytical working curve relating DPPD concentration to fluorescence intensity was obtained with the use of a device which consisted of a fluorescence cell, a bulb where the DPPD solution was kept during the degassing operations, and a valve for connection to the vacuum line. All solutions were degassed by three freeze-pump-thaw cycles. The excitation wavelength was $300 \mathrm{~nm}$ and the fluorescence intensities at $380 \mathrm{~nm}$ for $\mathrm{n}$-heptane solutions of DPPD were measured to establish the analytical working curve.

The extraction of DPPD from polyethylene films was carried out with the use of an extraction cell shown in Figure 2. It consisted of a fluorescence cell, a solvent chamber, an extraction chamber, and a valve for connection to a vacuum 1 ine.

At the beginning of an extraction experiment, a quantity of $n$-heptane arid DPPD-polyethylene films were placed in a thoroughly cleaned extraction cell according to the following procedure. A teflon-coated stirring magnet and *Certain commerical materials and equipment are identified in this paper to adequateiy specify the experimental procedure. This identification does not imply recommendation or endorsement by the National Bureau of Standards, nor does it imply that the material or equipment identified is necessarily the test available for the purpose. 


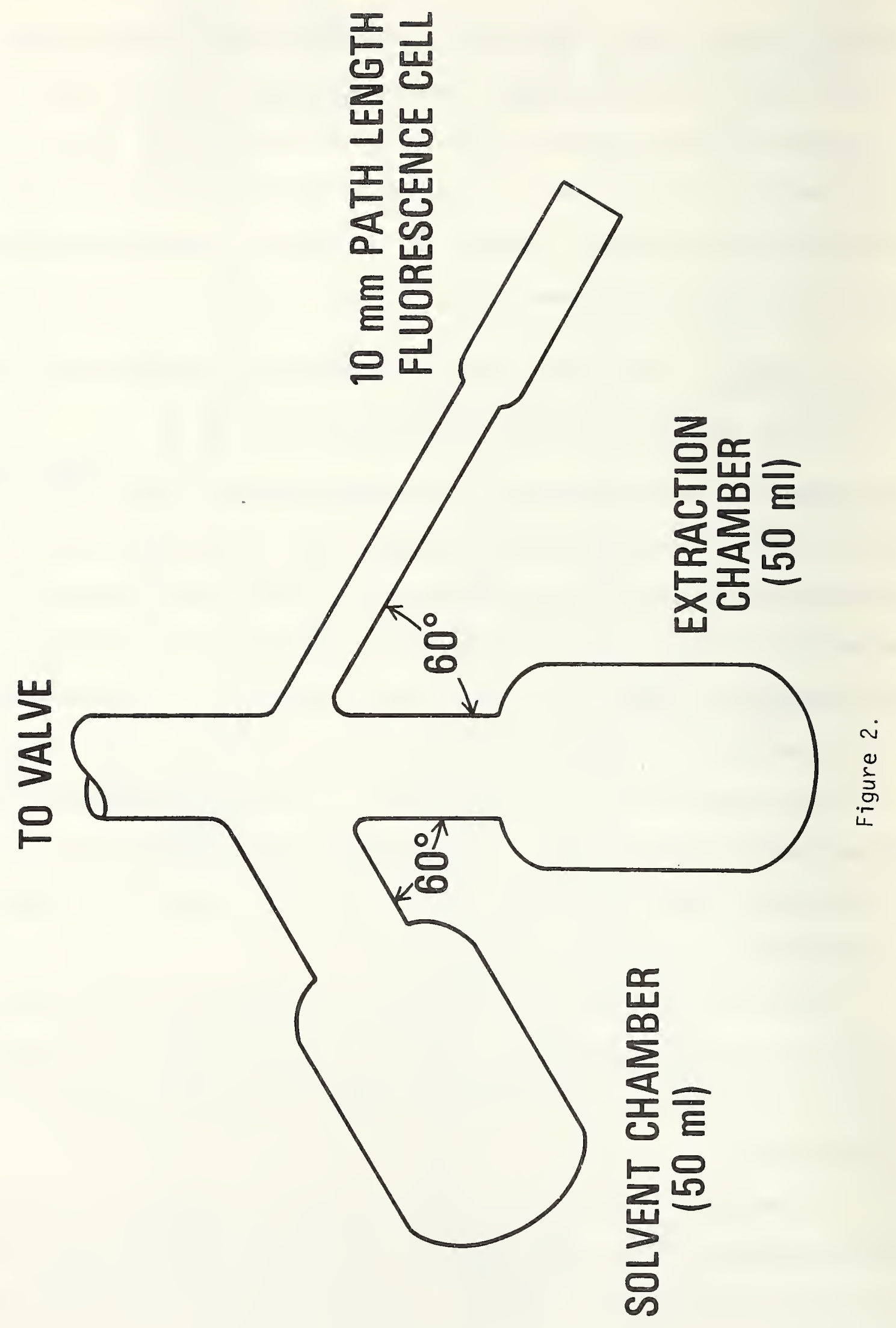


seven DPPD-polyethylene films weighing 0.343 gram were placed in the extraction chamber. After the extraction cell was evacuated with a vacuum line equipped with a diffusion pump, 7.2 grams of $n$-heptane (previously degassed at other part of the vacuum line) was distilled into the solvent chamber. Then the valve was closed and the extraction cell was taken to a $22.0^{\circ} \mathrm{C}$ water bath where its lower part including the two chambers and the fluorescence cell was immersed in the bath water and allowed to equilibrate at $22.0^{\circ} \mathrm{C}$. At the beginning of the extraction experiment, all the $n$-heptane was poured into the extraction chamber, the lower part of the extraction cell was reimmersed in the $22.0^{\circ} \mathrm{C}$ water bath, and the magnetic stirrer was turned on. At various elapsed times, some $n$-heptane solution was poured into the fluorescence cell and its fluorescence intensity at $380 \mathrm{~nm}$ was measured with an Aminco-Bowman spectrofluorimeter whose cell holder was kept at $22.0^{\circ} \mathrm{C}$. The solution was then poured back to the extraction chamber for additional extraction. 


\section{Results and Discussion}

$N, N^{\prime}$-diphenyl-p-phenylenediamine (DPPD)-

(a) Thermal Property - From measurements with a differential scanning calorimeter on DPPD samples in nitrogen atmosphere, we concluded that DPPD may crystallize in two forms, one with the melting point at $146.4{ }^{\circ} \mathrm{C}$ and the other with the melting point at $151.6^{\circ} \mathrm{C}$. The DPPD we purified was mostly in the lower-melting form. However, the relative amounts of the two forms may be altered by thermal treatment. Povet'eva and coworkers ${ }^{1}$ reported a melting point of $147{ }^{\circ} \mathrm{C}$ for a DPPD sample recrystal1ized from $\mathrm{n}$-heptane while Nespurek and Tlustakova ${ }^{2}$ reported a melting point of $152{ }^{\circ} \mathrm{C}$ for a DPPD sample recrystallized from ethanol.

(b) Fluorescence Property - Figure 3 shows the fluorescence spectra of a DPPD solution in $n$-heptane, the solid curve and the dashed curve being for the solution after and before degassing, respectively. The concentration of DPPD was $14.3 \mathrm{ppm}$. Degassing not only increased the overall fluorescence intensity but also made detectable fluorescence below $340 \mathrm{~nm}$ which was completely quenched by oxygen. We do not know yet whether the latter fluorescence is an inherent characteristic of the DPPD-n-heptane system or it is due to some impurities.

Analytical Working Curve - Figure 4 shows the relative fluorescence intensity F of DPPD-n-heptane solution as a function of DPPD concentration $\mathrm{C}$ in ppm. These data for fourteen solutions have been fitted to the equation

$$
F=131 c-1.6 c^{2} \text {. }
$$

The standard deviations for the first and the second coefficients are 3.7 and 0.4 , respectively. 


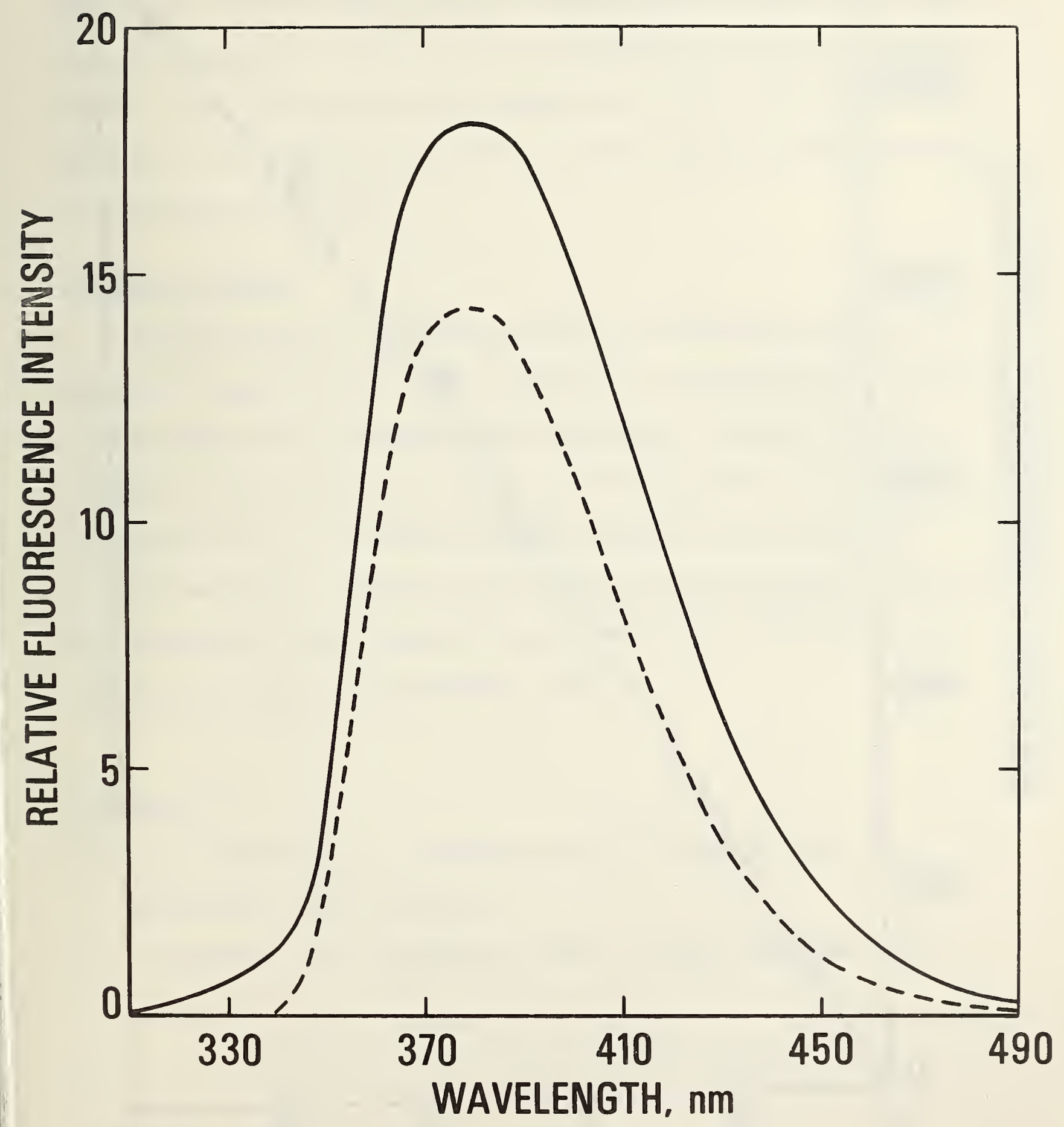

Figure 3. 


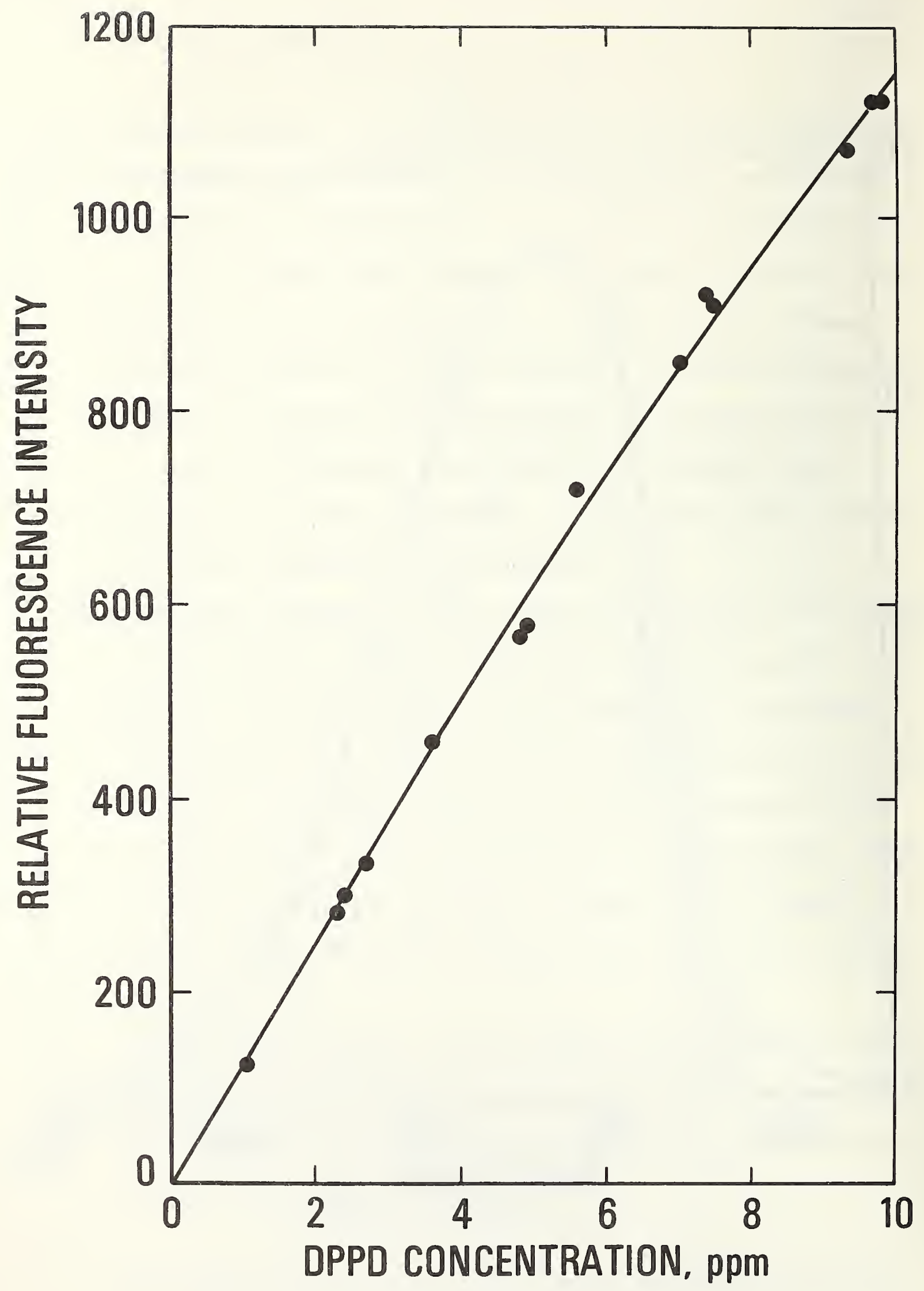

Figure 4. 
Migration of DPPD From Polyethylene Films

A preliminary result of the extraction of DPPD-polyethylene films with well-stirred $n$-heptane at $22.0{ }^{\circ} \mathrm{C}$ is shown in Figure 5 . There, the DPPD concentration of the solution in the extraction chamber is plotted as a function of $t^{1 / 2}$, where $t$ is the elapsed time in minutes. From the initial slope of the plot, we have obtained $D=2.8 \times 10^{-9} \mathrm{~cm}^{2} / \mathrm{sec}$ as an estimate of the diffusion coefficient of DPPD in these polyethylene films at experimental conditions.

\section{Concluding Remarks}

The degradation of antioxidants during film-forming processes and extraction experiments has been an obstacle to the measurements of antioxidant migration by the fluorescence technique. We have developed a procedure that overcomes this obstacle and have applied it to measure the migration of $N, N^{\prime}$-diphenyl-p-phenylenediamine from low-density polyethylene films. Additional experiments are being pursued to confirm the preliminary result described here, and to measure the migration of other antioxidants from polyethylene films.

\section{References}

1. Z. P. Povet'eva, L. A. Chetkina, and V. V. Kopylov, Sov. Phys. Crystallogr., 21, 168 (1976).

2. S. Nespurek and M. Tlustakova, Czech. J. Phys., B21, 1225 (1971). 


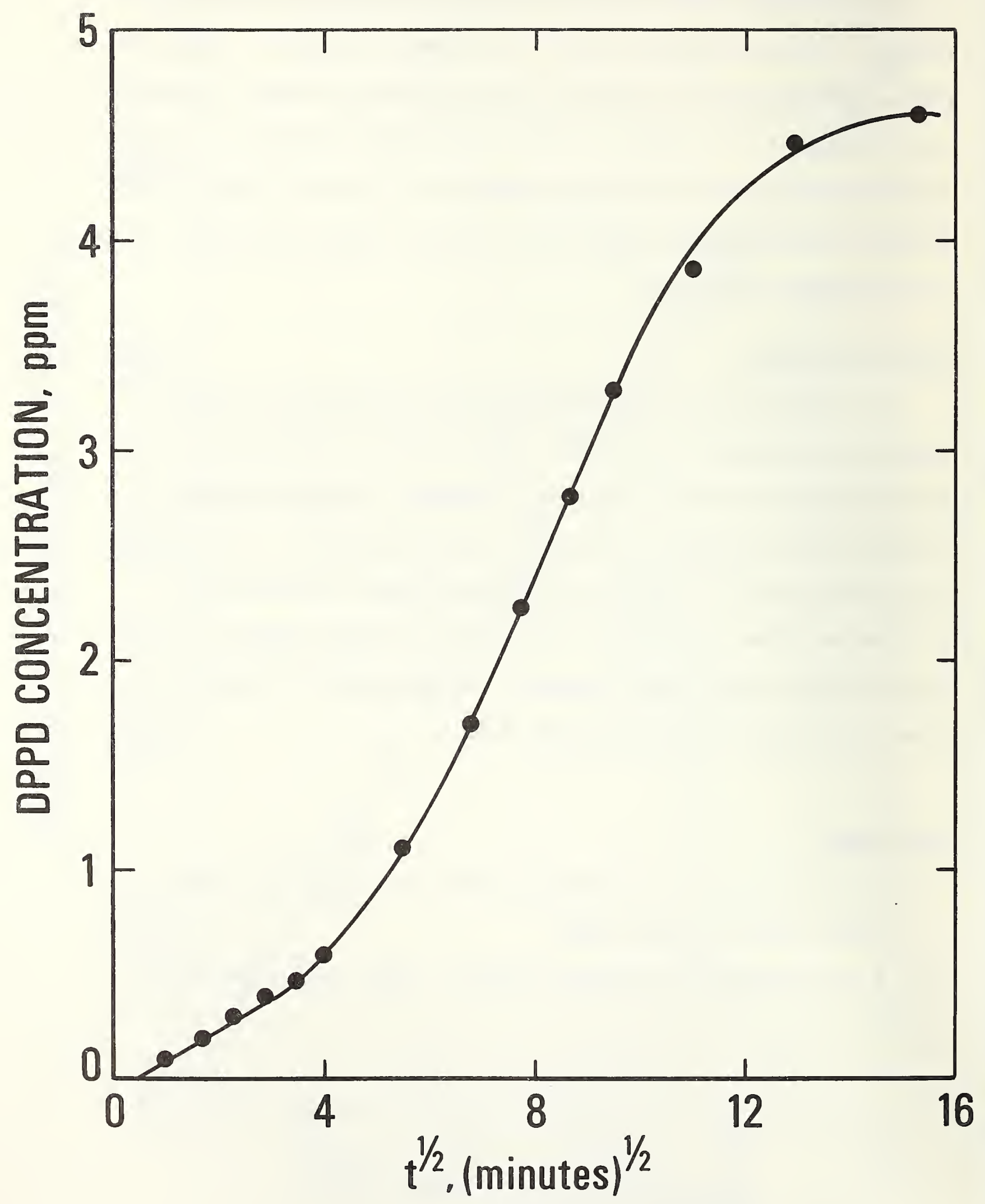

Figure 5 
SECTION VI

Experimental Measurement of Migration by Extraction 


\section{INTRODUCTION}

Diffusion coefficients of low molecular weight migrants moving from polymeric materials into surrounding liquid media under well stirred conditions are measured in this laboratory for the following combinations of base polymer, migrant, migrant concentration, solvent and temperature:
Polymer
Linear Polyethylene (LPE), SRM 1475
Branched Polyethylene (BPE), SRM 1476
Polypropylene (PP)
Migrants
$n$-Octadecane, $\mathrm{n}-\mathrm{C}_{18} \mathrm{H}_{38}$
n-Dotriacontane, $\mathrm{n}-\mathrm{C}_{32} \mathrm{H}_{66}$
BHT or 3,5-di-t-buty1-4-hydroxytoluene

Migrant Con- From less than $100 \mathrm{ppm}$ to greater than $10 \%$

centration

Solvents Accelerating solvents: $n$-heptane, n-octadecane,

Fat and 0il Simulating Solvents: Corn 0il, Ethanol, n-Octanol Tributyrin, Trioctanoin, HB307

Aqueous Solvents: Water, Ethanol-Water Mixtures

Temperatures $24,30,60^{\circ} \mathrm{C}$

Except experimental works on polypropylene and extractions involving $n-$ octanol and HB307 as solvents, most experiments are now completed. Therefore, in this report we present mostly empirical correlations from the large sets of data being collected in the past two years.

\section{EXPERIMENTAL}

\section{Materials}

The characteristics of the base polymers and of the base radioactive labeled migrants are listed in Table $\mathrm{TA}$ and $\mathrm{IB}$ respectively. 
TABLE TA

Characteristics of Polyolefin Samples

\begin{tabular}{|c|c|c|c|}
\hline & $\begin{array}{l}\text { Linear } \\
\text { Polyethylene } \\
\text { NBS-SRM } 1475\end{array}$ & $\begin{array}{c}\text { Branched } \\
\text { Polyethylene } \\
\text { NBS-SRM } 1476\end{array}$ & $\begin{array}{c}\text { Isotactic } \\
\text { Polypropylene } \\
\text { Pro-fax } 6301\end{array}$ \\
\hline$M_{n}$ & $18.310(\mathrm{GPC})$ & & \\
\hline$M_{W}$ & $53,070(\mathrm{GPC})$ & & 290,000 \\
\hline$M_{W}$ & $52,000(L S)$ & & \\
\hline$M_{w} / M_{n}$ & & & 11 \\
\hline $\mathrm{CN}$ & 0.890 & 0.8132 & \\
\hline TCB & 1.010 & 0.9024 & \\
\hline DHN & 1.180 & 1.042 & 2.1 \\
\hline
\end{tabular}

Melt Flow

2.07

1.19

Rate, g/10 min.

Isotacticity, \%

$95-96$

$$
\begin{aligned}
& \text { GPC - Gel Permeation Chromatography } \\
& \text { LS - Light Scattering } \\
& \text { CN - I-chloronaphthalene } \\
& \text { TCB - 1,2,4-trichlorobenzene } \\
& \text { DHN - decahydronaphthalene, Decal in } \\
& t=130^{\circ} \mathrm{C} \text { for polyethylenes } \\
& t=135^{\circ} \mathrm{C} \text { for polypropylene }
\end{aligned}
$$

Melt Index by Procedure A, ASTM Method D 1238-65T, Test Condition D, $190^{\circ} \mathrm{C}$, load $325 \mathrm{~g}$ for SRM 1475 and $2160 \mathrm{~g}$ for SRM 1476.

Density by ASTM Method D 1505-67; sample prepared by Procedure A, ASTM Method D 1928-68.

Isotacticity is determined as fractional insoluble in Decalin at room temperature after entire sample has been dissolved at $160^{\circ} \mathrm{C}$ and allowed to cool.

*Certain commercial materials and equipment are identified in this paper to adequately specify the experimetnal procedure. This identification does not imply recommendation or endorsement by the National Bureau of Standards, not does it imply that the material or equipment identified is necessarily the best available for the purpose. 
TABLE TB

Characteristics of Radioactive Tracers

$$
\begin{aligned}
& \mathrm{n}-\mathrm{C}_{18} \mathrm{H}_{38}{ }^{-1-{ }^{14} \mathrm{C}} \\
& \mathrm{n}-\mathrm{C}_{32}{ }^{\mathrm{H}} 66^{-16,17-}{ }^{14} \mathrm{C}
\end{aligned}
$$$$
\mu \mathrm{C}_{\mathbf{j}} / \mathrm{mg}
$$$$
\mathrm{ng} / 25 \mathrm{dpm}
$$

13.3

0.13

0.83

3,5-di-tert-butyl-4hydroxytoluene- $7-{ }^{14} \mathrm{C}$

45.5

0.25

57.9

0.19 


\section{Sample Plaque Preparation}

The following procedure for the mixing of additives to the polymer stock and the molding of the sample plaques was chosen. A large quantity of polyethylene powder stock was prepared from either NBS-SRM 1475 or 1476 pellets first by dissolution in hot toluene or xylene. Most of the polyethylene precitates out upon cooling. The precipitate, together with the residue obtained by evaporationg the solvent, was dried in a vacuum oven to remove the last trace of solvent.

To a quantity of the polyethylene powder stock, a specific amount of labeled additive dissolved in a highly volatile solvent is mixed. The mixture is then evaporated to dryness in a rotary evaporator, together with a number of glass beads to act as a ball mill, under reduced pressures at relatively low temperatures and further dried in a vacuum oven.

The mixture is then compression molded in a hydraulic press operated at about $180^{\circ} \mathrm{C}$ for 01 igomers and $165^{\circ} \mathrm{C}$ for BHT. Plaques of $125 \mathrm{~mm} \times 125 \mathrm{~mm}$ or less are molded with brass or stainless steel shim stocks of appropriate thickness sandwiched between two sheets of teflon or teflon coated plates. The teflon surfaces are used for the easy removal of the sample plaques without the aid or contamination of mold release agents.

The isotactic polypropylene was received in a granular form from the supplier without any additives. Therefore, it was used directly to form a slurry with the migrant in solution as mentioned above. Experimental Methods

Two extraction methods were used, i.e., (1) continuous extraction into limited solvent volume and (2) discrete extraction into simulated infinite solvent volume. 
In method (1) an extraction vial of $25 \mathrm{ml}$ in volume with a teflon valved cap is used. The solvent in the vial will only meet glass walls and the teflon surfaces during normal experimental processes. A silicon plug is situated above the valve. A small area of the silicon rubber, less than $1 \mathrm{~mm}$ in diameter and used as a septum for the hypodermic needle, may be exposed to the solvent vapor. The polymer sample may sometimes be surrounded by a nichrome or stainless steel screen to prevent it from sticking to the walls or another sample has lower density than the solvent.

The total amount extracted, $M_{t}$, at time $t$ is

$$
M_{t}=C_{s t} W_{s t}+\sum_{i=1}^{t-1} C_{s i} W_{a i}
$$

where $C_{s}, W_{s}$ and $W_{a}$ represent the concentration of the migrant, total weight of the solution (including that of the aliquot) and the weight of the aliquot, respectively. At equilibrium the partition coefficient is estimated as

$$
k=\frac{C_{S \infty}}{C_{p \infty}}=\frac{C_{S \infty \infty}}{M_{0}-M_{\infty}}=\frac{C_{S \infty} W_{p}}{M_{0}-M_{\infty}}
$$

where $M_{0}$ is the amount of migrant originally present in the polymer of weight $W_{p}$, and $M_{\infty}$ is the total amount extracted at long times calculated from the concentration, aliquot weights and solution weights as mentioned above. The amount of migrant present in the aliquot is obtained via radioactivity indicated by liquid scintillation technique.

In method (2), the polymer sample is immersed in about $10 \mathrm{ml}$ of extracting solvent in a typical $20 \mathrm{ml}$ liquid scintillation counting vial. At specific times the sample is removed from the solvent, rinsed and placed in another vial with fresh solvent to repeat the extraction process. The total amount extracted at time $t$ is simply the sum of all extracts:

$$
M_{t}=\sum_{i=1}^{t} M_{i}
$$


Method (1) is able to yield information about the equilibrium partition coefficient at infinite extraction time. However, it suffers from rigid requirements of knowing accurately the ratio of aliquot versus total solution and of keeping track of materials lost during the sampling process for material balance purposes. As extraction time increases, there is only very small change in the concentration of extracted material in the solution, whereas the weighing or ratio error may persist. Therefore, the results at long time or high degree of extraction will show considerable degree of scatter.

Method (2) is much simpler in operation, but simulates a condition of migration into infinite media, and is relatively free from aforementioned experimental difficulties. However, it cannot be used to generate partitioning information nor migration kinetics for migrant that is sparingly soluble in the solvent. It should only be used for convenience when the migrant is highly soluble or miscible with the solvent.

For all the methods mentioned the extraction vials are shaken inside a temperature controlled aluminum block on a shaking table at a rate of about 200 reciprocations per minute.

When the extraction process is ended, radioactivity of the residual low molecular weight species remaining in the polyethylene sample is monitored by dissolving the sample in toluene at high temperatures. We found that the single crystals or precipitates of polyethylene in the counting vial does not interfere with the counting efficiency beyond the normal scattering of the counting results. 


\section{Experimental Run Designation}

In order to utilize the rather 7 imited graphical BASIC language resident in the graphics terminal, we have changed designations for each of the experiments into an all numerical representation as follows:

1) Least significant digit method of observation and repetition number.

2) Tens and Hundreds digits temperature in ${ }^{\circ} \mathrm{C}$.

3) Thousands and Ten Thousands digits solvent code.

4) Hundred Thousands and Millions digits sample code

$\begin{array}{ll}\text { N } & \text { Method or Repetition } \\ \text { TT } & \text { Temperature in }{ }^{\circ} \mathrm{C} \\ \text { SS } & \text { Solvent Code } \\ \text { PP } & \text { Polymer Code }\end{array}$

This coding scheme is described in more detail in Table $\mathrm{IC}$ and $1 \mathrm{D}$. 


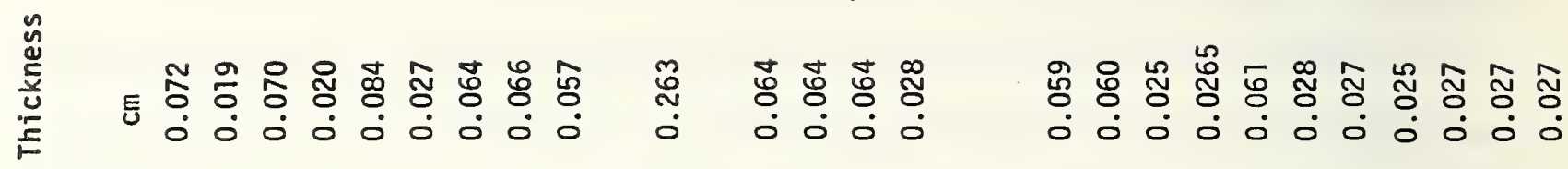

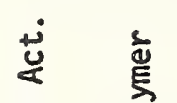

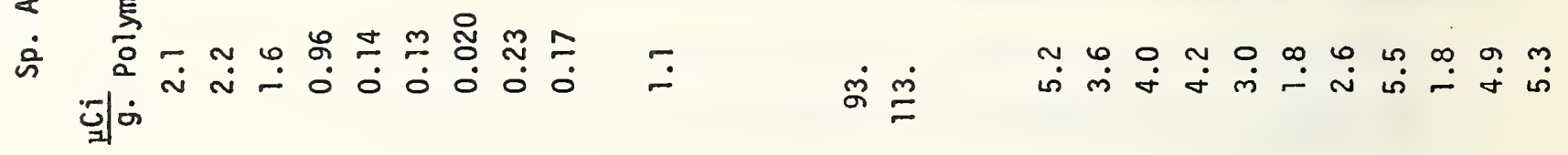

se

$\stackrel{\dot{0}}{0}$

$\stackrel{0}{\infty} \times x \times$

总 $-x \times x \times x \times x \times$

5 $x \times x \quad x \quad x \times x \times x$

$\frac{5}{0}$
ह
ह

‥

管 $x \times x \times$

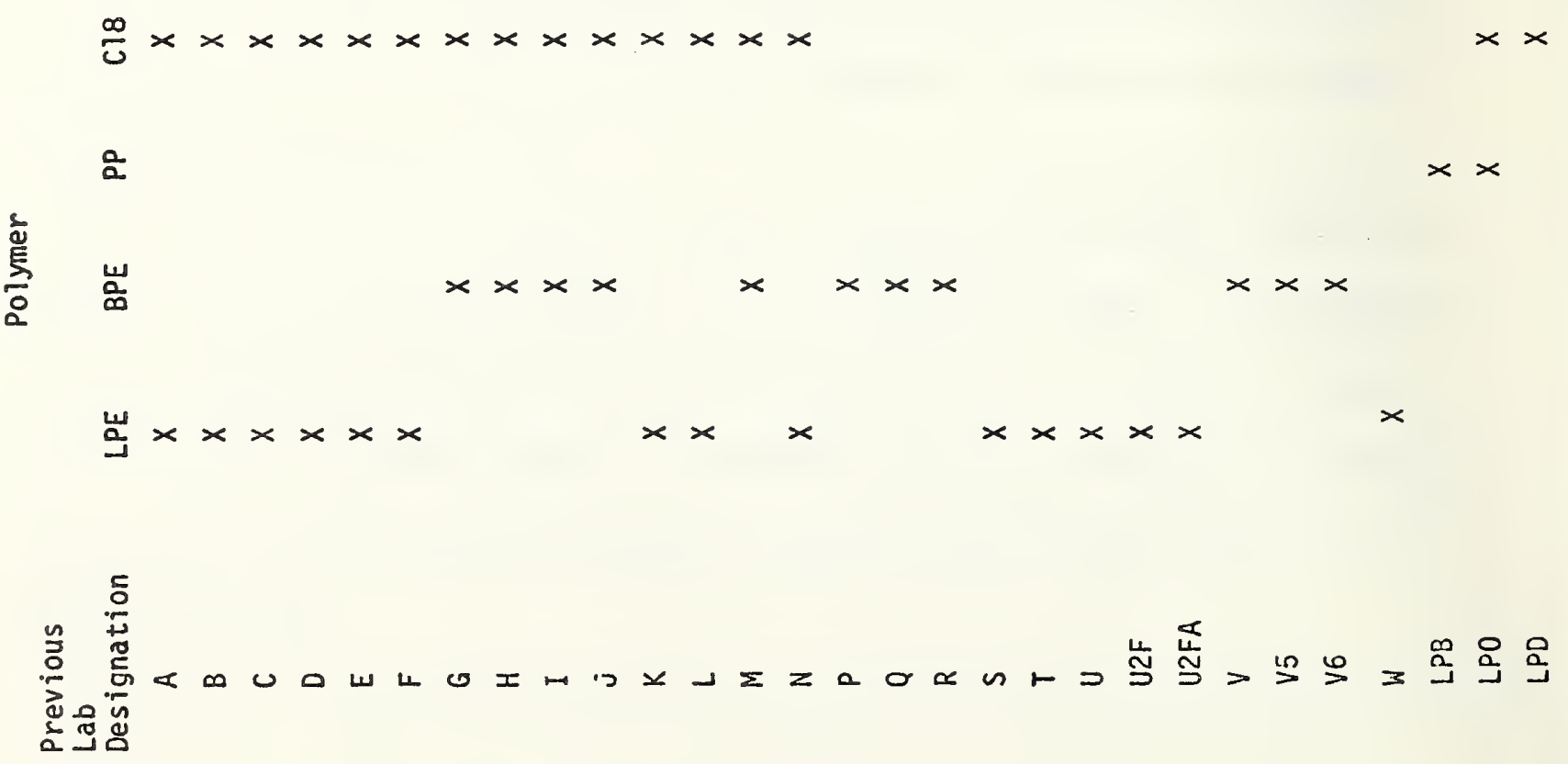

$x \times x \times x \times x$

$\stackrel{0}{\circ}$

$\frac{x}{\circ}-n m+\infty 6 n$ o 
Table 10

\section{$\times 10^{3}$ Solvent}

10, Corn 0i1

20, Ethanol

$21,10 \%$ Ethanol

23, 30\% Ethanol

25, 50\% Ethano 1

27 70\% Ethanol

29 90\% Ethanol

30 n-Heptane

40 n-Octadecane

50 n-Octanol

60 Tributyrin

70 Trioctanoin

80 Water $\times 10^{1}$ Temp.

$24,24^{\circ} \mathrm{C}$

$30,30^{\circ} \mathrm{C}$

$60,60^{\circ} \mathrm{C}$

\section{$\times 10^{0}$ Method}

0, Limited Solvent Volume

1, Unlimited Solvent Volume

$N$, Number of Repetitions 


\section{Estimation of Diffusion Coefficient}

One of the widely used solutions for the diffusion equation $\partial C / \partial t=D$ $\partial^{2} \mathrm{C} / \partial x^{2}$, solving for the case of diffusion between a plane sheet $p$ of thickness $2 l$ and a stirred liquid $s$ of finite volume $V_{S}$, is presented by J. $\operatorname{Crank}^{[1]}$

$$
\frac{M_{t}}{M_{\infty}}=1-\sum_{n=1}^{\infty} \frac{2 \alpha(1+\alpha)}{1+\alpha+\alpha^{2} q_{n}{ }^{2}} e^{-q_{n}^{2} T}
$$

where $\alpha=M_{s \infty} / M_{p \infty}=V_{s} / K V_{p}, K=C_{p \infty o} / C_{s \infty}$ and $T=D t / l^{2}$.

The solution for the non-zero positive roots, $q_{n}$, of

$$
\tan q_{n}=-\alpha q_{n}
$$

lies between $n \pi$ when $\alpha=0$ and $(n-1 / 2) \pi$ when $\alpha=\infty$. At $\alpha<<$,

$$
q_{n} \sim n /(1+\alpha) .
$$

For other values of $\alpha$,

$$
q_{n} \sim[n-\alpha / 2(7+\alpha)] \pi
$$

may be used as the starting value in a reiterative numerical computation.

Equation (1) converges rather slowly, thus enough terms must be used to avoid premature termination of the computation. At small values of $T(<<1)$, approximately $3^{-\log _{10} T}$ terms are required to reach a reasonable precision. Either of the two approaches described as follows may be used to simplify the computational effort.

1) Crank, J., "The Mathematics of Diffusion", Oxford University, Press, 1975. 


\section{Simplified Computation}

At small values of $\alpha(<<1)$, a master curve of $M_{t} /(1+\alpha) M_{\infty}$, which is equal to $M_{p t} M /$ so for absorption or $M_{s t} / \alpha M_{p o}$ for extraction, as a function of $T / \alpha^{2}$ may be constructed from equation (1) as listed in Table $2 \mathrm{~A}$. However, by Timiting the computations to $0.1 \geq T \geq 0.001$, the number of terms required will be 1 imited to about 5 at T 0.1 and about 45 at T 0.001 . The values of $M_{t} /(1+\alpha) M_{\infty}$ at smaller $\alpha$, but at corresponding $T / \alpha^{2}$, are set to equal to the values calculated for larger $\alpha$. The results of this selective computation deviates about $0.001 \%$ at T 0.1 from the more rigorous and tedious computations, and much less than $0.001 \%$ at lower $T$.

Table $2 \mathrm{~A}$ may be divided into three regions.

Region I, $T \leq 0.1$. The system is far from equilibrium, $M_{t} /(1+\alpha) M_{\infty}$ is a function of $T / \alpha^{2}$ oniy.

Region II, $5 \geq T \geq 0.1$. The system is approaching equilibrium, detailed computation must be carried out for different a values (only 2 to 6 terms are required for equation (1) in this region). The results of the computation for the regions approaching equilibrium are listed in Table 2B. For $\alpha<<1$ and $T / \alpha^{2}>10^{3}, M_{t} /(1+\alpha) M_{\infty}=1-\alpha / \sqrt{\pi T}$ before reaching equilibrium.

Region III, T $\geq 5$. For all practical purposes, equilibrium has been reached with the deviation $\delta=1-M_{t} / M_{\infty} \leq 10^{-T}$, where $M_{\infty}=M_{s 0} /(1+\alpha)$ in the case of absorption and $M_{\infty}=M_{p o} \alpha /(1+\alpha)$ in the case of extraction. For $\alpha<1$, equilibrium may be reached much earlier (Table 2B).

\section{Alternate Approximation}

An alternative form of the solution $[1]$

$$
M_{t} / M_{\infty}=(1+\alpha)\left\{1-e^{T / \alpha^{2}} \operatorname{erfc}\left(T^{1 / 2} / \alpha\right)\right\}
$$




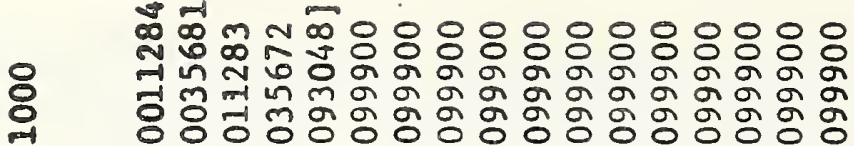

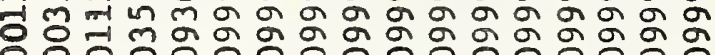

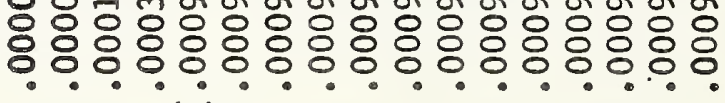

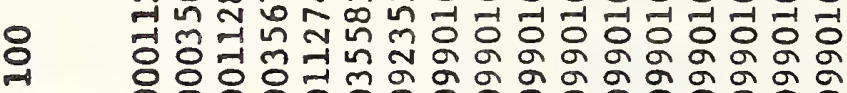

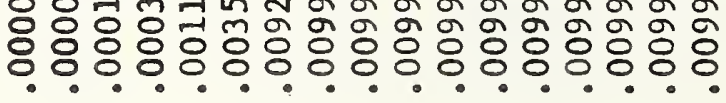

$\infty \mathrm{m}^{\mathrm{N}} \mathrm{N}$

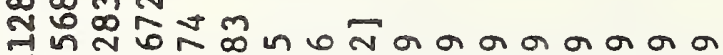

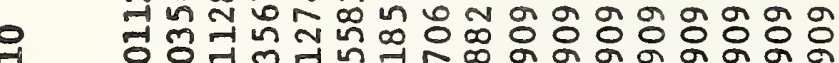

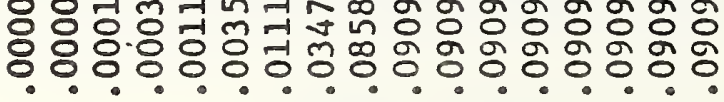

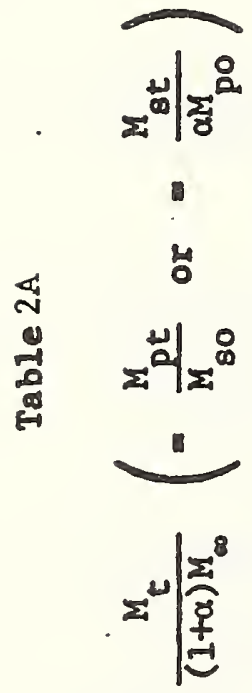

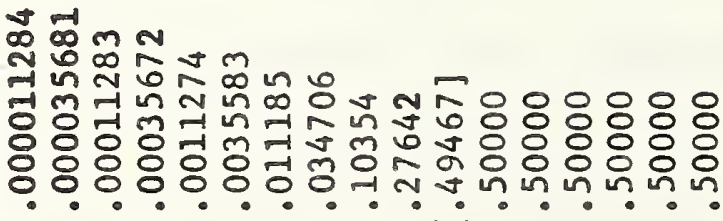

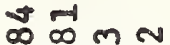

N

$\stackrel{-1}{0}$

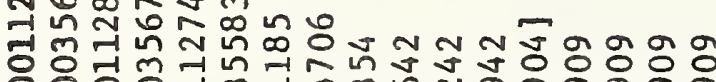
유유.

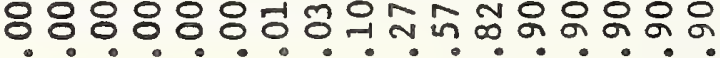

-

एँ

叫

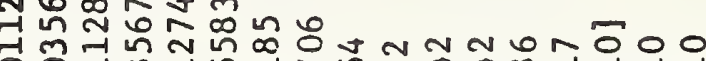

品年

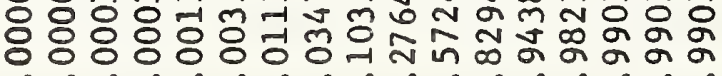

政

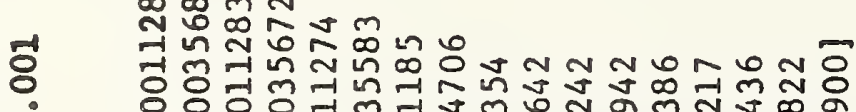

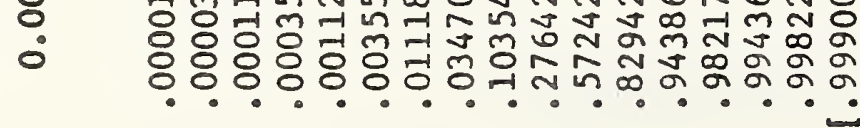

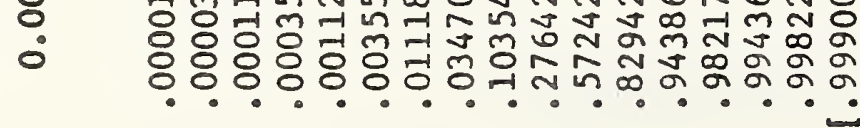

$\int_{E \rightarrow O}^{\infty}$

일

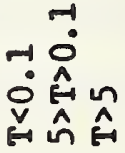

-iं่

등 등 등 


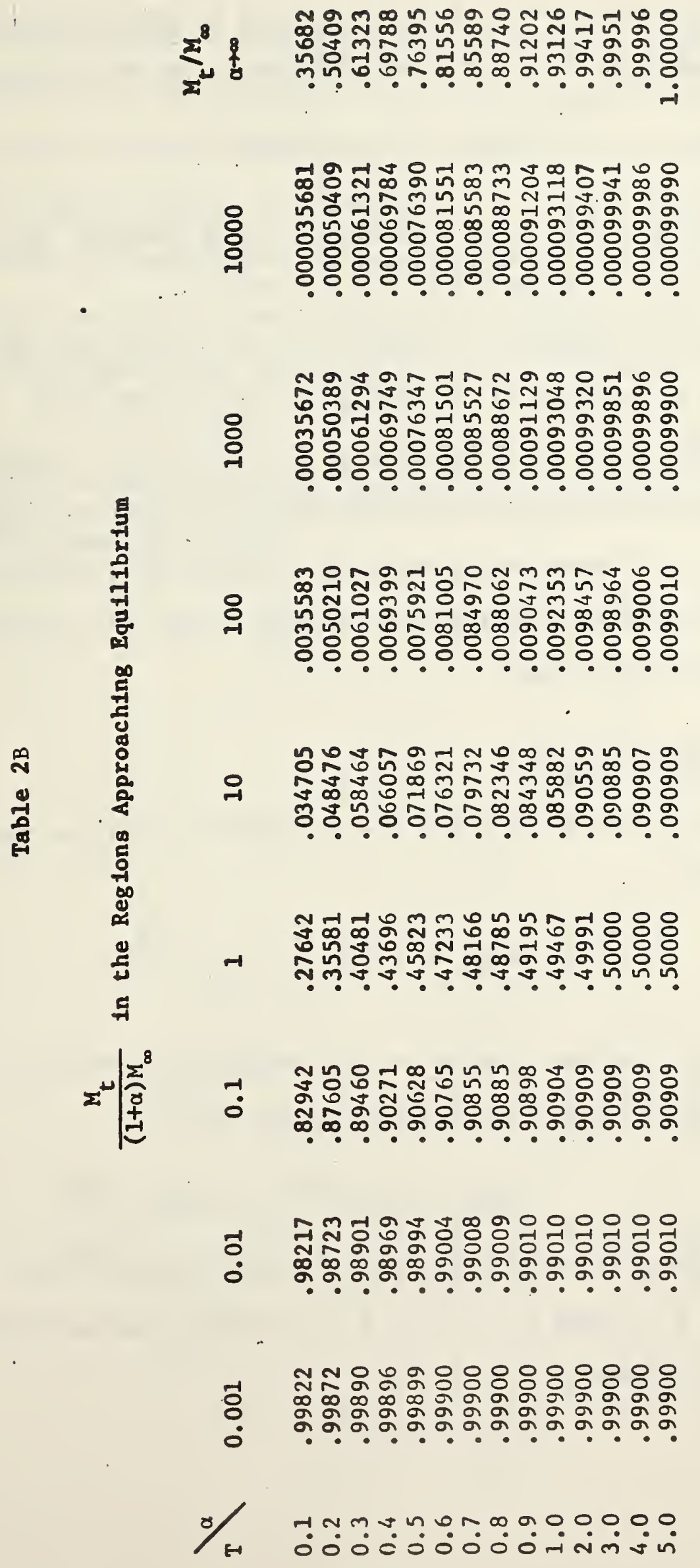


may be used in some cases and is relatively simple in computation. One of the rational approximations ${ }^{[2]}$ for the error function yields the following

$$
M_{t} /(1+\alpha) M_{\infty}=1-\sum_{n=1}^{5} a_{n} \tau^{n}+\varepsilon
$$

where $\tau=1 /\left(1+0.3275911 T^{1 / 2} / \alpha\right), a_{1}=0.254829592, a_{2}=-0.28449636, a_{3}=1.421413741$, $a_{4}=-1.453152027, a_{5}=1.061405429$, and $|\varepsilon|<1.5 \times 10^{-7}$.

At $\alpha<<1$, results computed from equation (2) or (3) deviates less than 0.00001 from that of equation (1) at $T / \alpha^{2}<5$ or at $M_{t} /(1+\alpha) M_{\infty}<0.75$. Maximum deviation of 0.0035 occurs at $T / \alpha^{2} \sim 500$ or at $M_{t} /(1+\alpha) M_{\infty} \sim 0.97$. Therefore, equation (2) or (3) may be used to generate the master curve for equation (1) at $\mathrm{T} \leq 0.1$ (Region I). However equation (2) or (3) does not yield any information about the region approaching equilibrium at larger values of $\alpha$, and hence should be used with discretion.

\section{Computation for Infinite Bath}

when $\alpha \rightarrow \infty, M_{t} / M_{\infty}$ approaches a limit, and equation (1) can be reduced to a function of T only,

$$
M_{t} / M_{\infty}=1-2 \sum_{n=1}^{\infty} \frac{1}{q_{n}^{2}} e^{-q_{n}^{2} T}
$$

where $q_{n}=(n-1 / 2) \pi$. Values of $M_{t} / M_{\infty}$ in the range $5 \geq T \geq 0.1$ are also 1 isted in Table 2. For $T \leq 0.1, M_{t} / M_{\infty}$ is a linear function of $T^{1 / 2}$.

$$
M_{t} / M_{\infty}=2(T / \pi)^{1 / 2}=1.128379 T^{1 / 2}
$$

Deviations of equation (5) from equation (4) is less than $10^{-10}$ at $T<0.05$, about $10^{-6}$ at $T=0.1$, about $5 \times 10^{-4}$ at $T=0.2$ and becomes much greater than 0.0 at $T=0.3$ or higher. 
By combining the use of Equation (2) or (3) at $T \leq 0.1$ (Region I) and the use of Equation ( 1 ) at $T \geq 0.1$ (Region II and III), computational requirements for the solutions of the diffusion equation between a plane sheet and a well stirred liquid may be reduced to a minimum.

The results of computations are shown graphically in Figure $1 A$, where $T / \alpha^{2}$ is used as the abscissa and in Figure $1 B$, where the more familiar reduced time $T=D t / \ell^{2}$ is used as the abscissa, for various values of $\alpha$ as noted along the curves.

By regressive means, diffusion coefficient at any point may be estimated from the above computation method. 


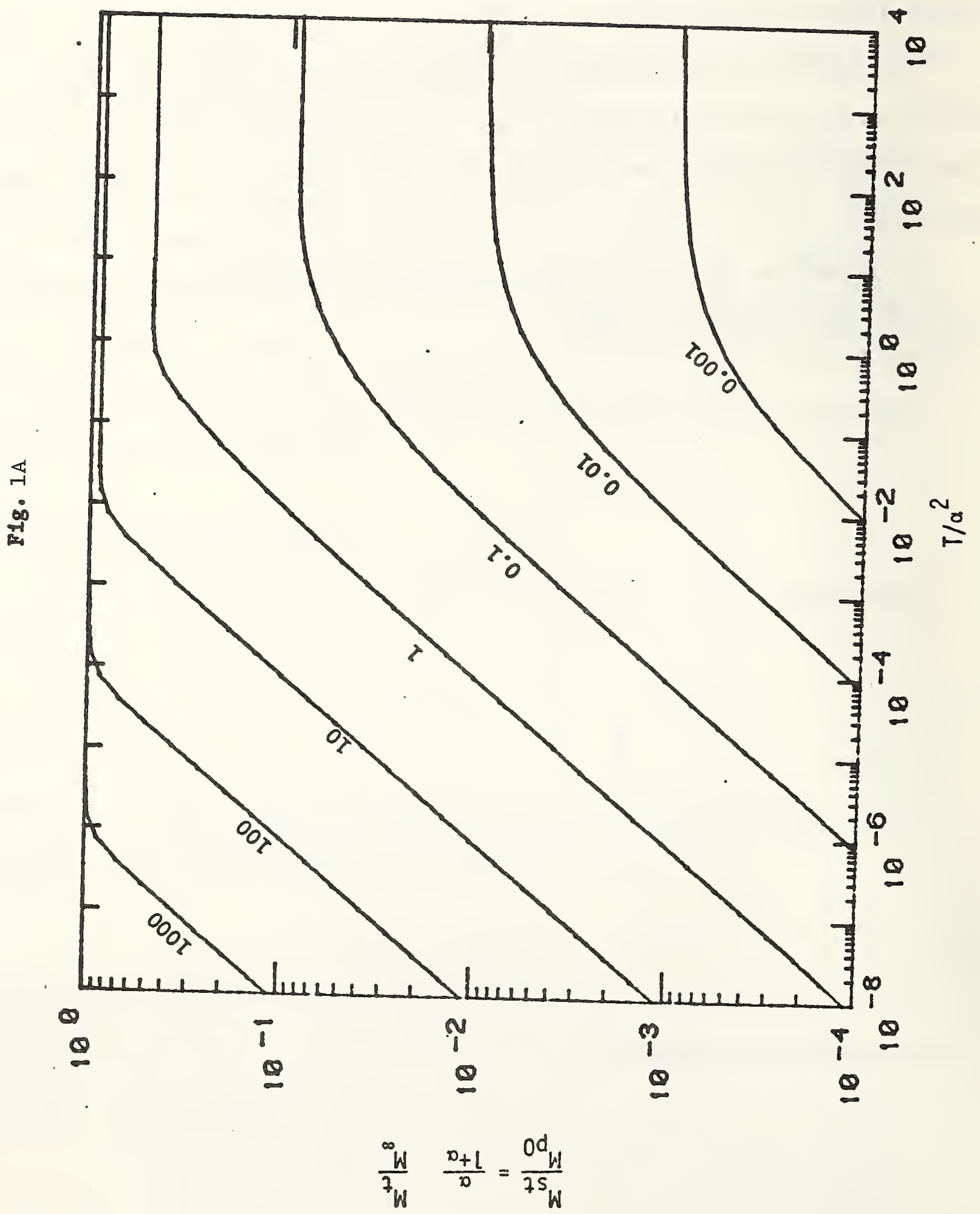




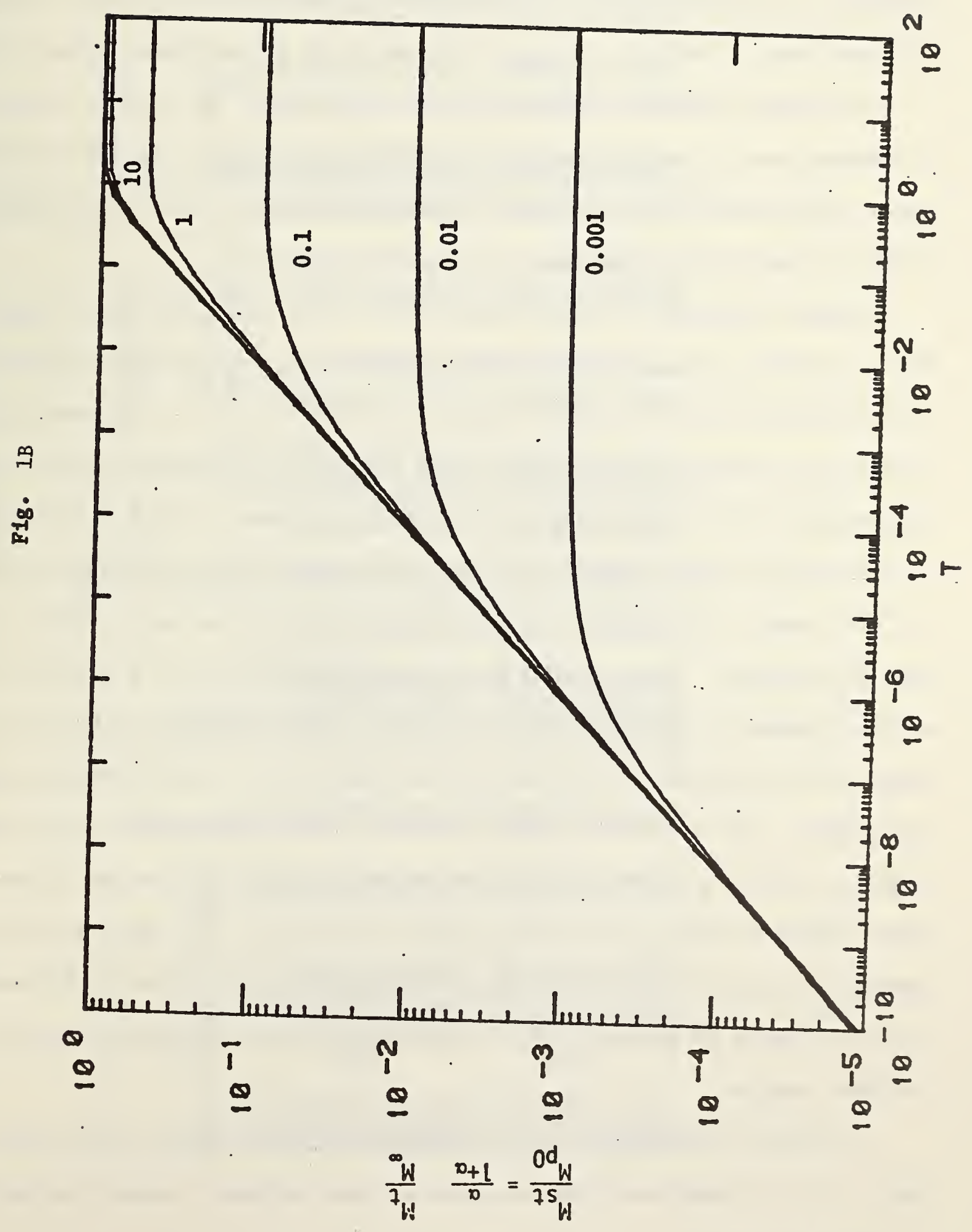




\section{Results of Extraction Experiments}

The results of extraction experiments are summarized in Table $3 \mathrm{~A}$ through $3 \mathrm{I}$ for the extraction of n-octadecane by triglycerides, by ethanol, by ethanol/water mixtures, by $n$-heptane and by $n$-octadecane, of $n$-dotriacotane and of BHT by triglycerides, by ethanol, ocatanol and water and by n-heptane, respectively.

The tables provide information on the test pieces on to their weight, thickness $L$, exposed area A, specific activity in $\mu \mathrm{Ci} / \mathrm{g}$ of polymer, amount extracted at the end of experiment $M_{f} / M_{0}$, the amount of solvent absorption and the maximum of diffusion coefficient observed.

Special problems are encountered in the preparation of sample plagues with BHT as additive. A much longer time is required to mix BHT with polymeric powder in a rotating flask with glass beads acting as a ball mill. Apparently, BHT is relatively insoluble in the polymer even in the molten state therefore, sample plagues made from insufficiently mixed batch of powder mixtures yield autoradiographs of distinctive sharp regions of streaks and patches. Each molding at $185^{\circ} \mathrm{C}$ seems to "fix" some $15-20 \%$ of BHT, i.e. only $80 \%$ of the BHT may be extracted exhaustiveiy even by $n$-heptane. When plagues being remolded at $185^{\circ} \mathrm{C}$ for 5 times, only $20 \%$ of original amount of BHT would be extractable. The remainders of the BHT are combined with the polymer. The residual radioactivities remain with the powderous precipitate, even after the plagues were dissolved in toluene at high temperatures. However, molding at $165^{\circ} \mathrm{C}$ of well mixed batches of BHT and polymeric powder seem to offer rather uniformly distributed sample plagues with less than $5 \%$ of BHT being combined with the polymer. The $M_{f} / M_{0}$ of $n$-heptane extractions of BHT samples, as listed in Table $3 G$ through $3 I$, give indication of the extractable fraction of BHT in those samples.

In order to provide quick cross references the diffusion coefficients from Table 3 are arranged according to migrant, then polymers, temperature and solvents in Table $4 \mathrm{~A}$ to $4 \mathrm{D}$. 


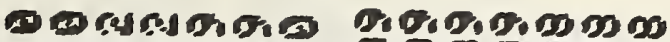

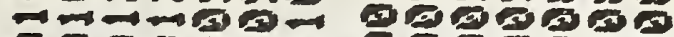

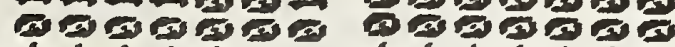

$$
\begin{aligned}
& 111111111111
\end{aligned}
$$

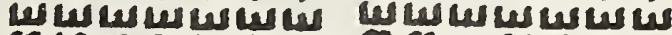

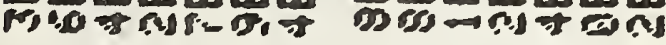

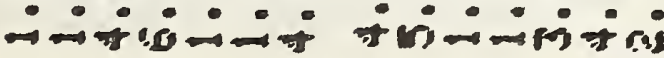

$$
\begin{aligned}
& \frac{2}{9} \text { gs }
\end{aligned}
$$




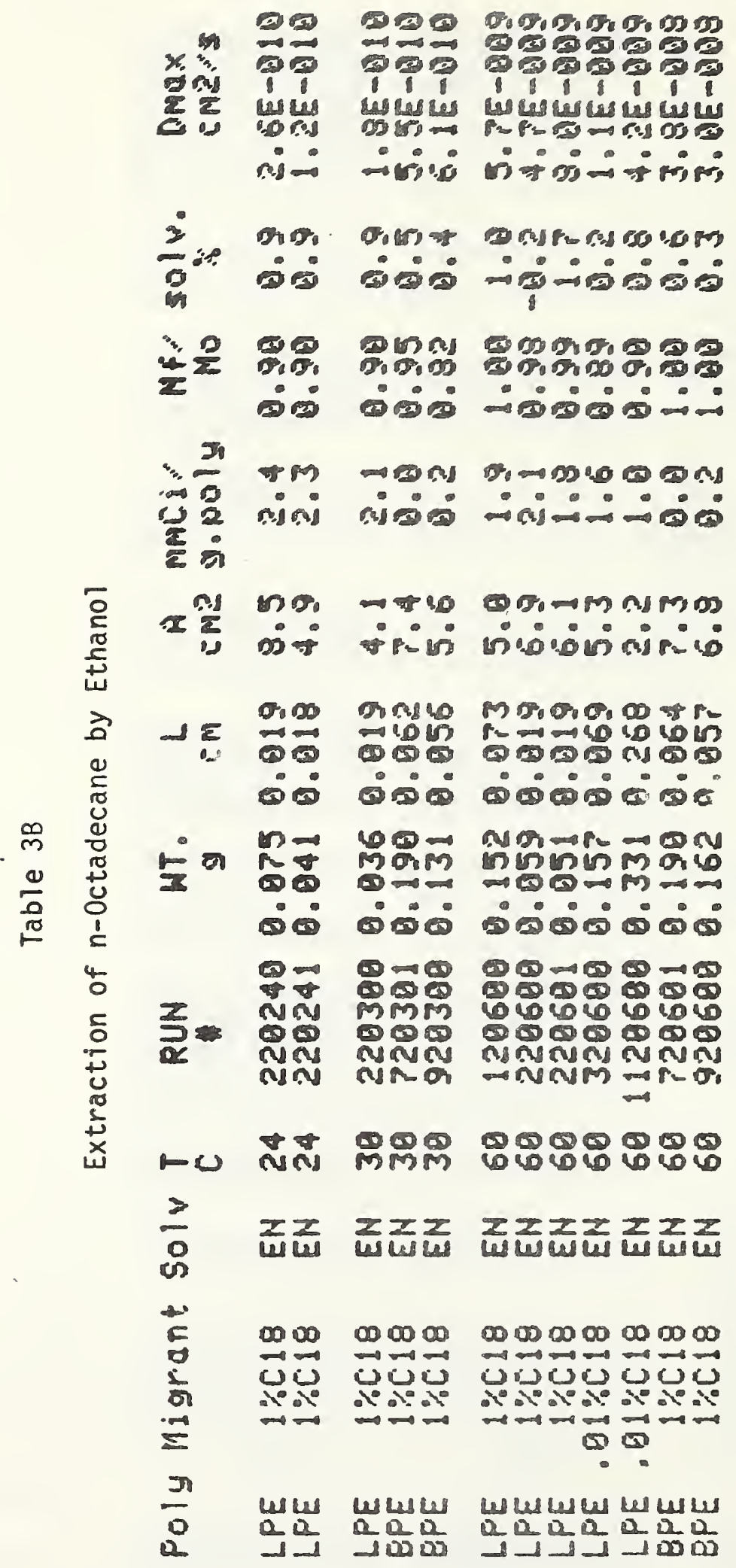




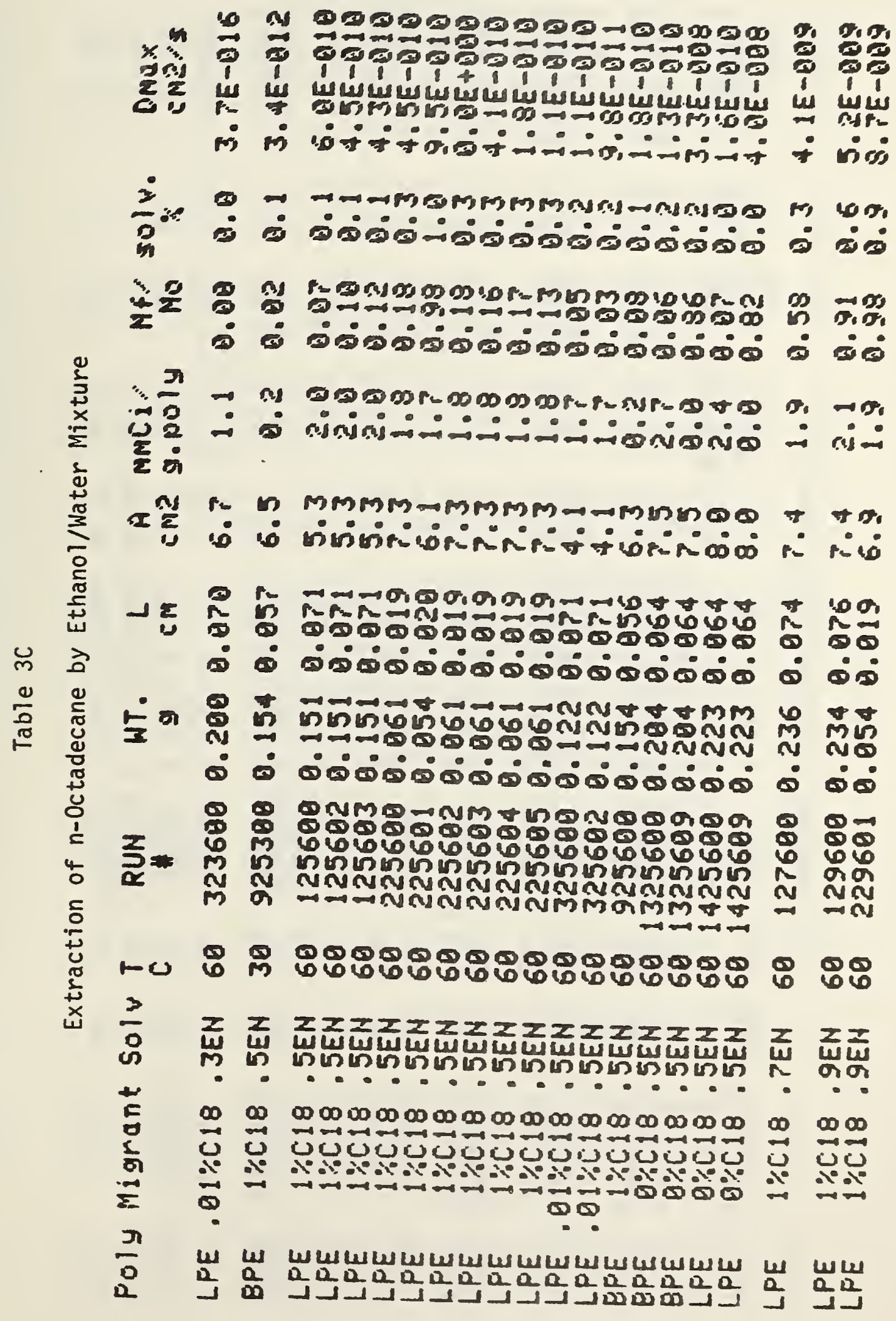




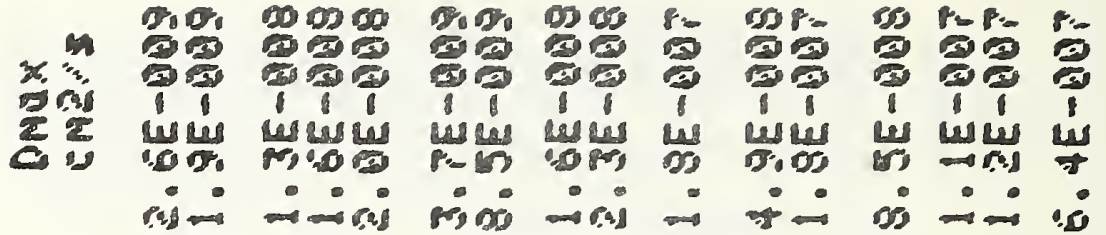

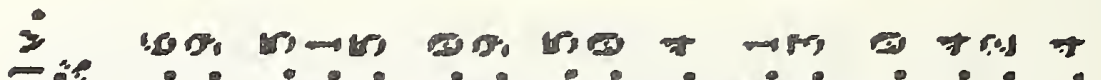

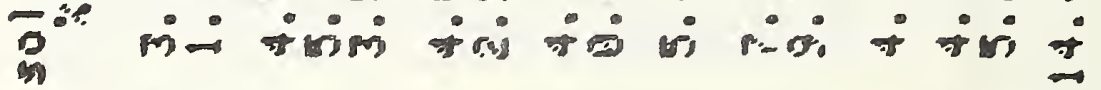

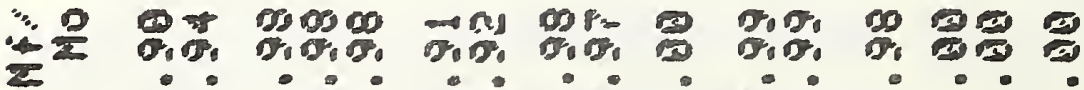

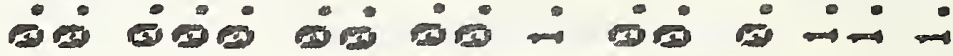

$$
\begin{aligned}
& \text { I }
\end{aligned}
$$

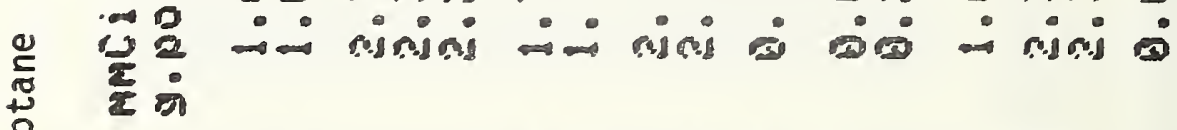

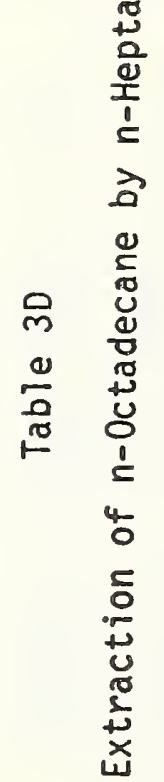

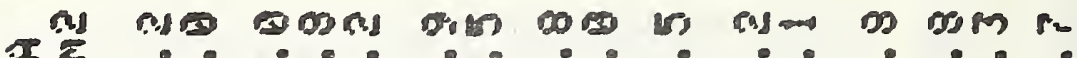

$$
\begin{aligned}
& \text { is एंi }
\end{aligned}
$$

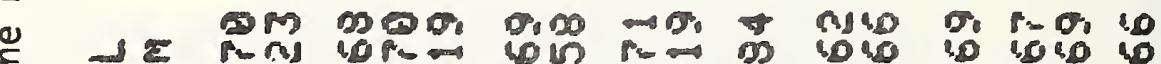

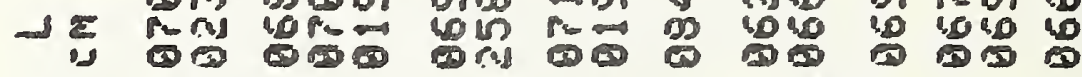

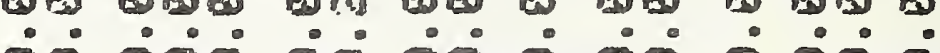

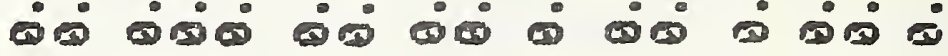

$$
\begin{aligned}
& \therefore \pi \text { D }
\end{aligned}
$$

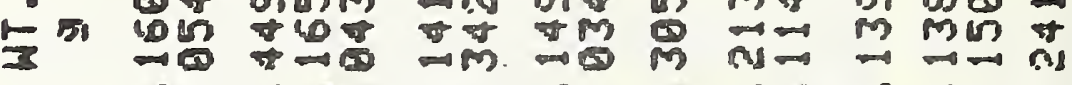

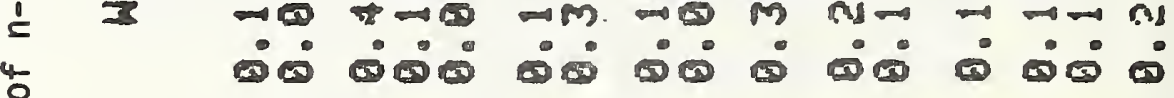

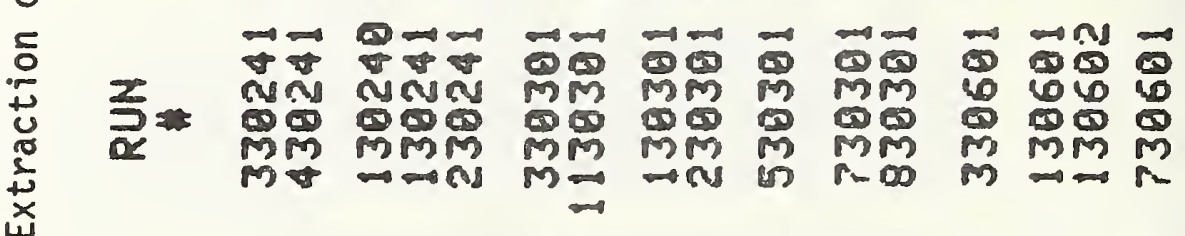

$$
\begin{aligned}
& \text {-U Nल }
\end{aligned}
$$

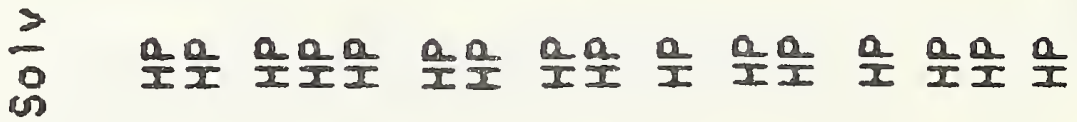

$$
\begin{aligned}
& \begin{array}{llllllllllll}
\hline & 0000 & 000000 & 0000 & 0000 & 00 & 0000 & 00 & 0000 & 00
\end{array}
\end{aligned}
$$

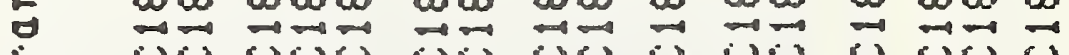

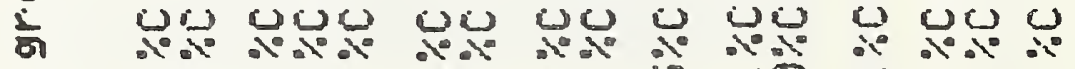

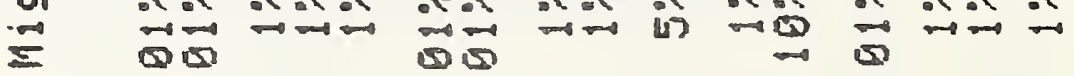

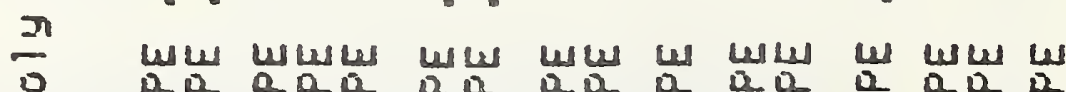

$$
\begin{aligned}
& \text { a a a a a a a a a a a a a a a a }
\end{aligned}
$$




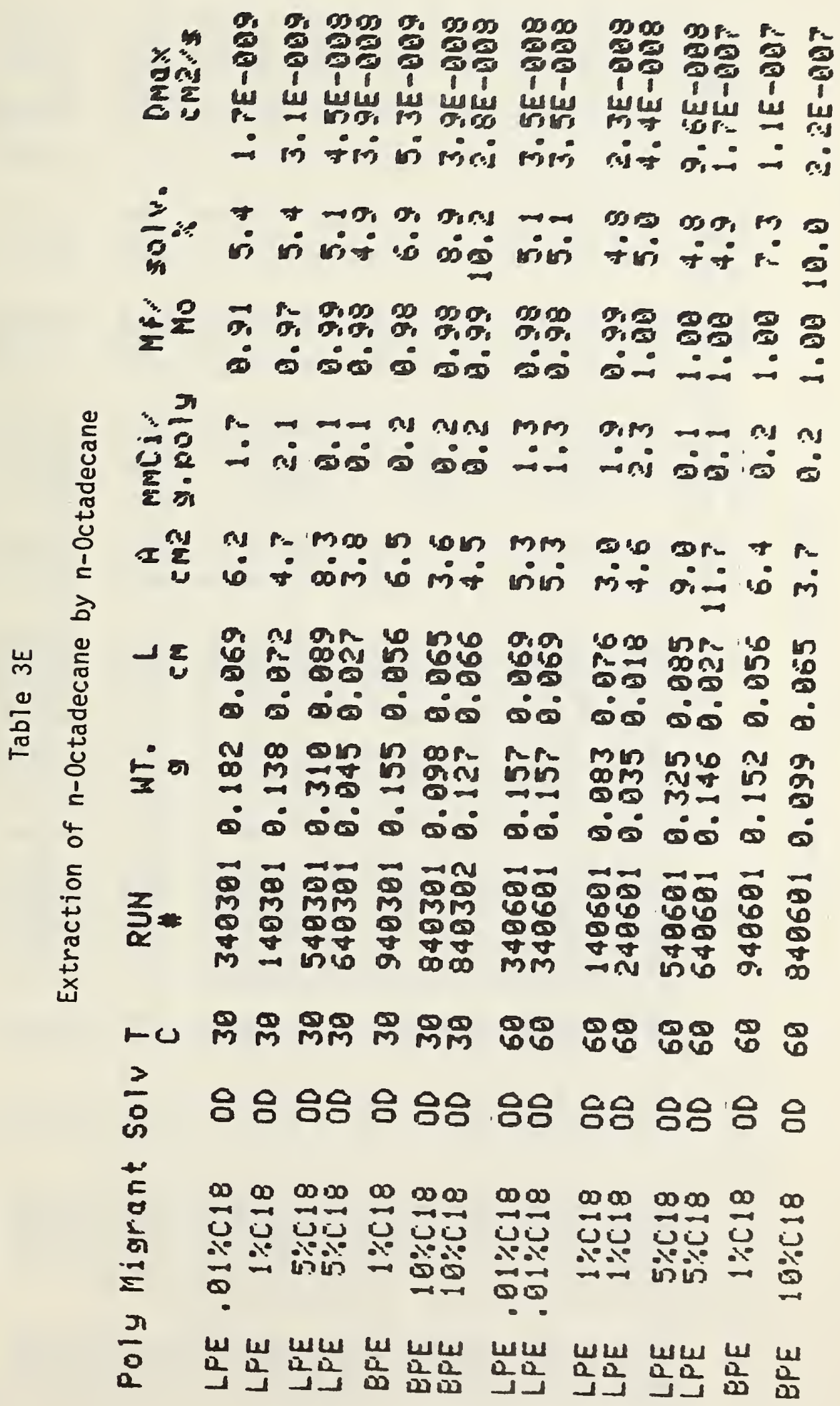




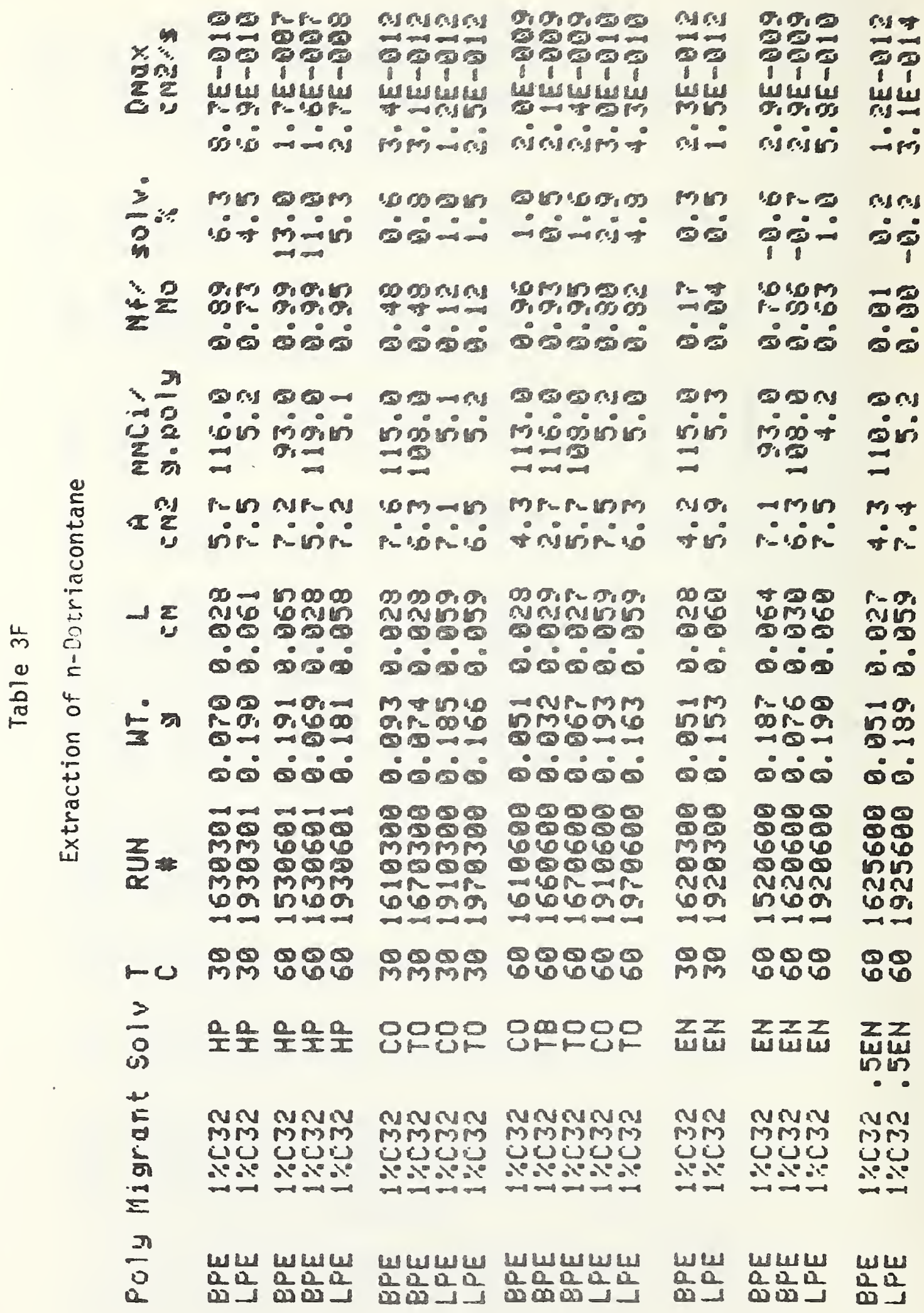




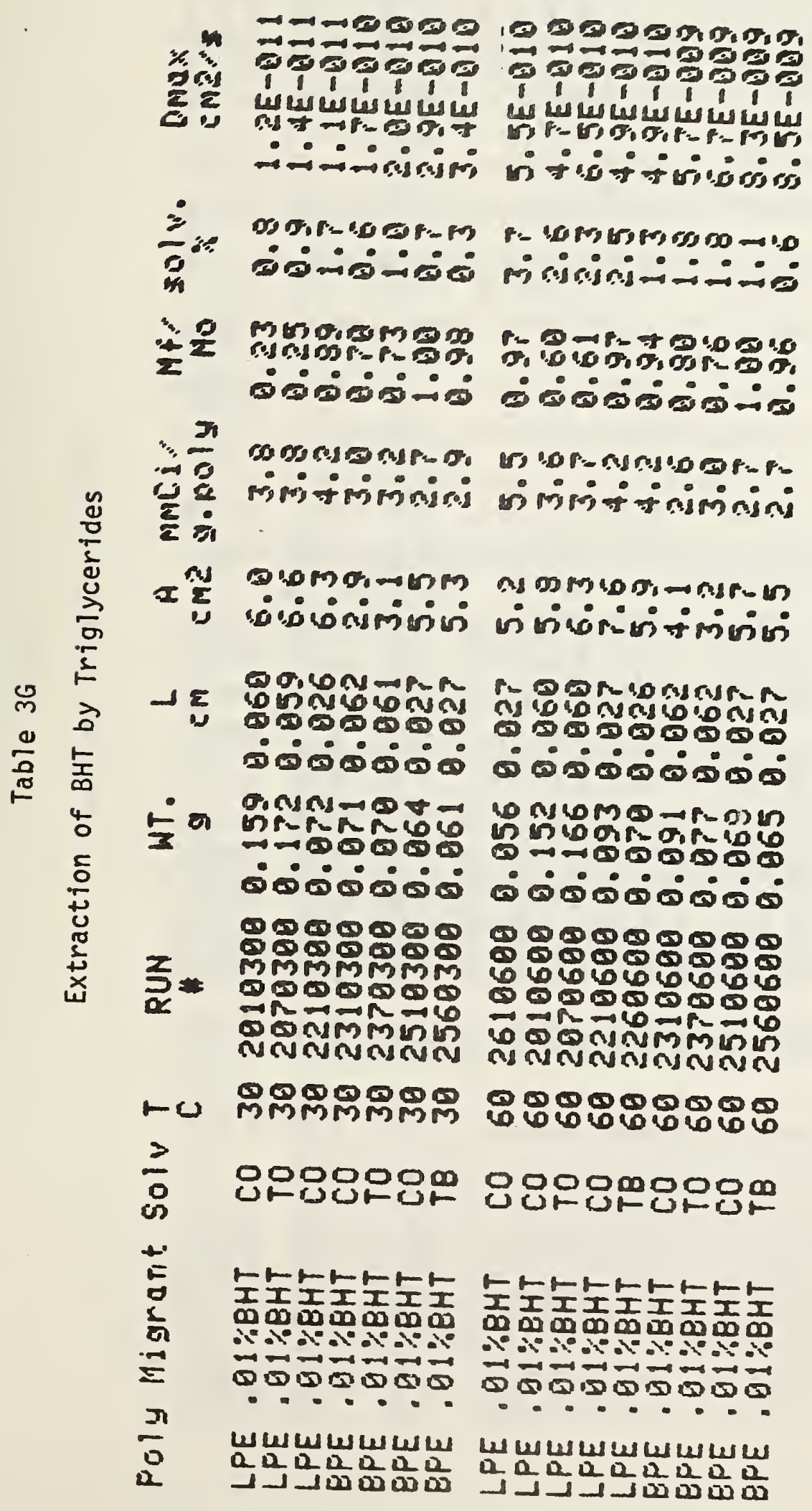




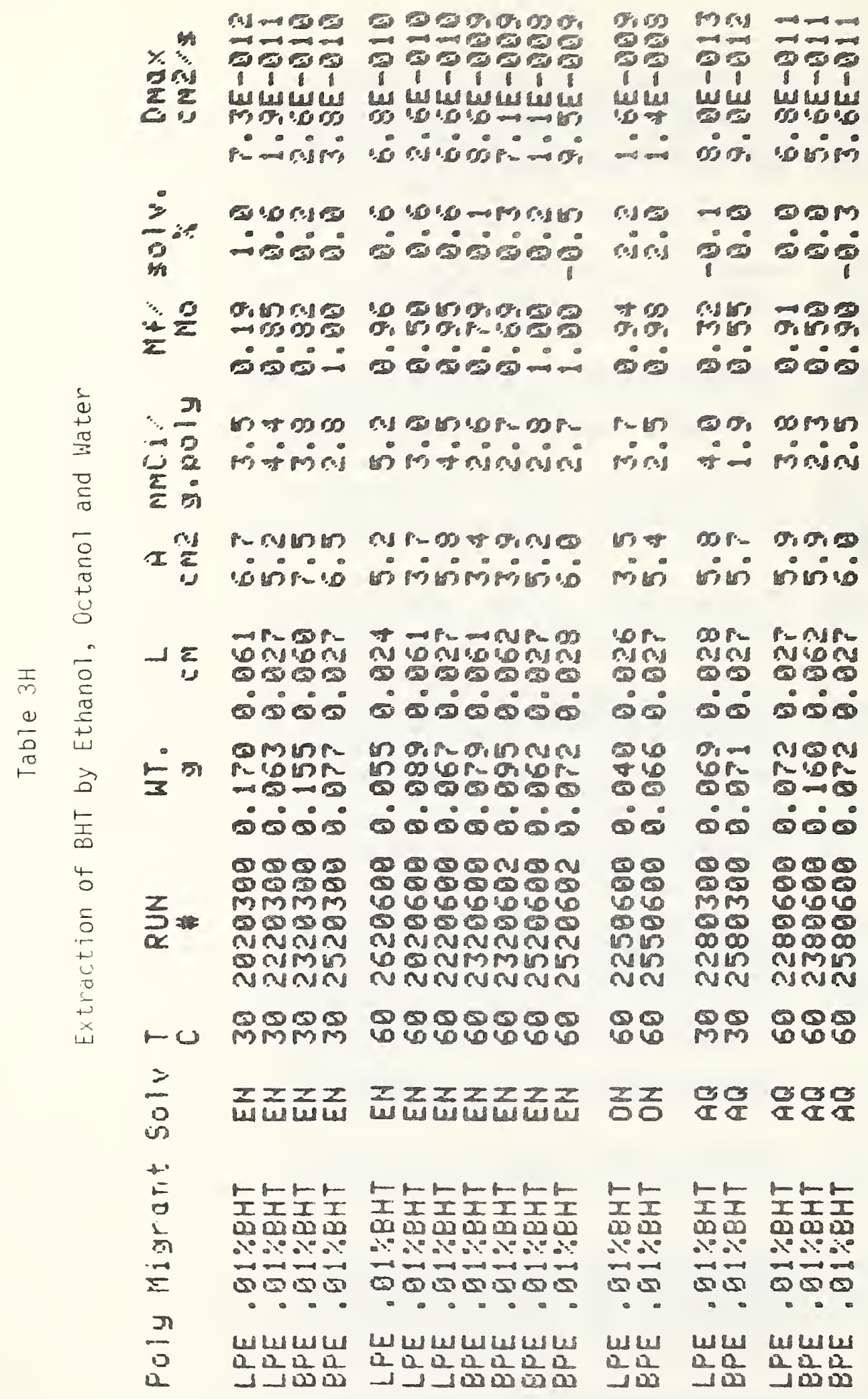


0000000000

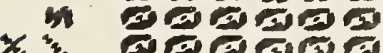

x.

$2 \div$ usentures

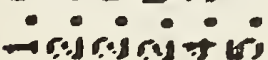

; $\cos c 000=$

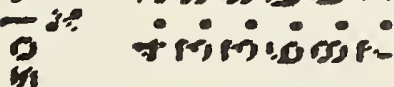

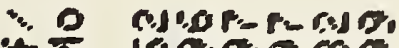
$2=00 \% 0000 \%$ $z$

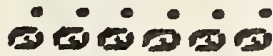
$\because \frac{\pi}{2} \cos a s-\sigma_{0} \cos$

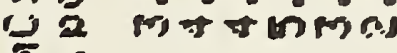
$\approx 2$

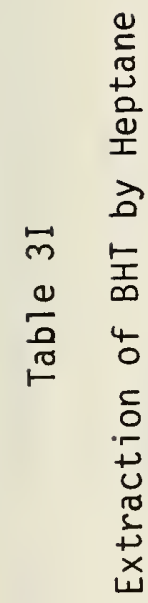

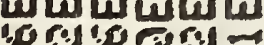

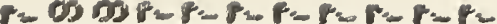

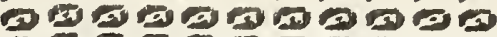

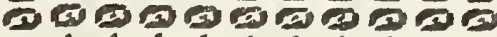
1 I I I I I I

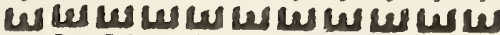
$\Rightarrow$ fo r.s

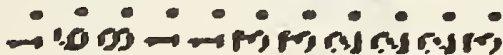

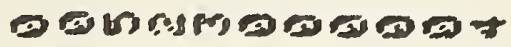

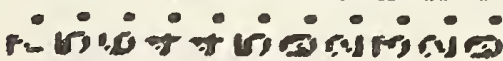
r- ogr

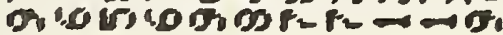

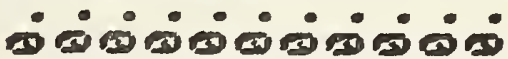

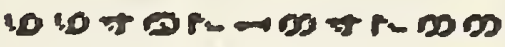

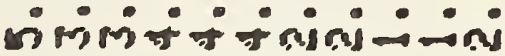

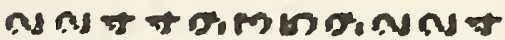

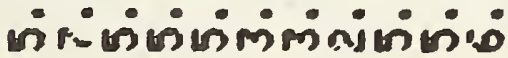

$n=-40.0 \mathrm{M} M-100 \mathrm{rm}$ M to sorimlom.

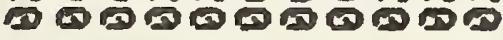
๓ ம்

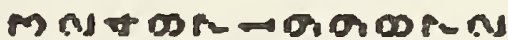
in orm rn

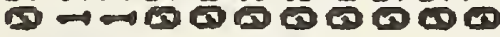
ஸं $\rightarrow \infty \circ$ $-60-\infty$ 9000 $\frac{\Sigma}{\alpha=1}$ MMMMMM a 00000 MMMMMM ond amin ำก NNN 000000

-U MMMMMM

$\rightarrow=N \rightarrow-\infty M-N=$ 600000000600

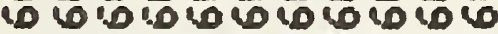
06000000000 MMMMMNMM MM $100-n M m M$ के

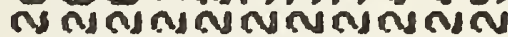

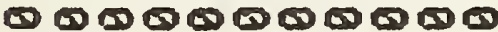
10 106001061060

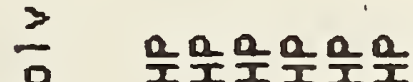

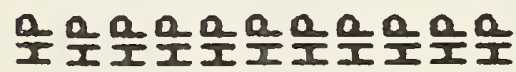
Е 000000000

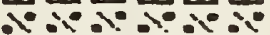

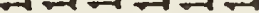
๑心⿴囗十心

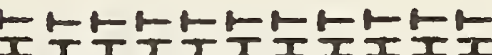
13 ต $x^{0} x^{\circ} x^{\circ} x^{\circ} x^{\circ} x^{\circ} x^{\circ} x^{\circ}$

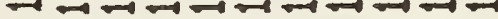
\$

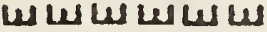
2. 2 a 20

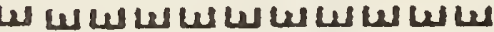

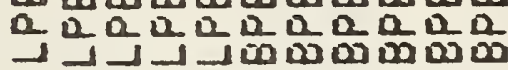




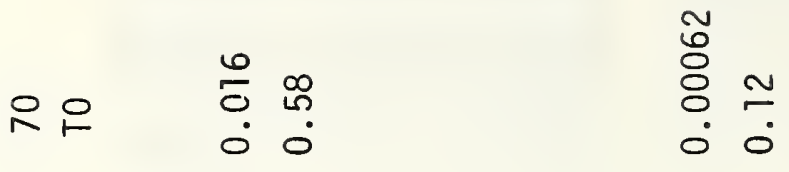

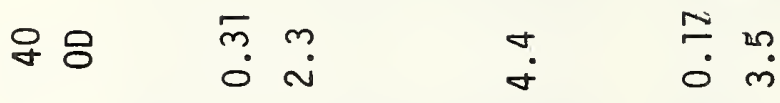

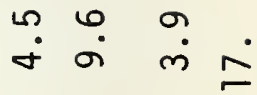

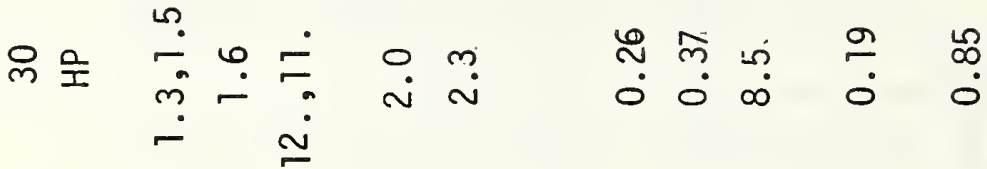

$$
\begin{aligned}
& \stackrel{\infty}{\infty}
\end{aligned}
$$

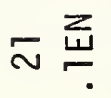

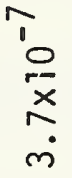

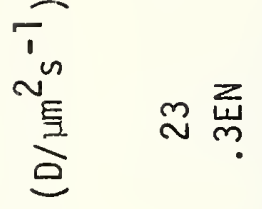

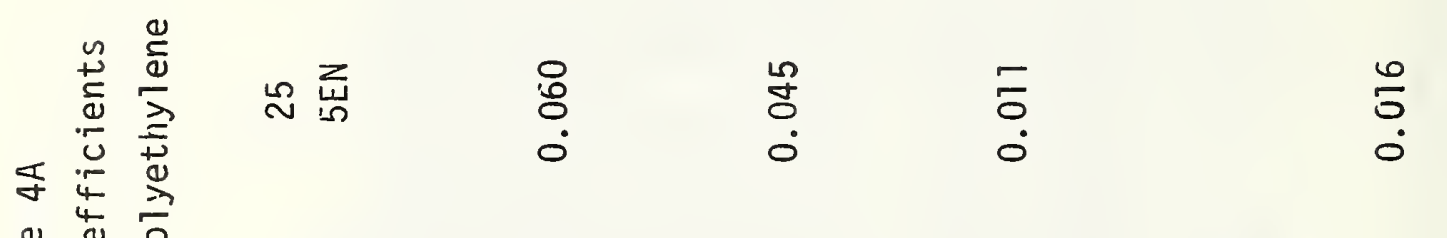

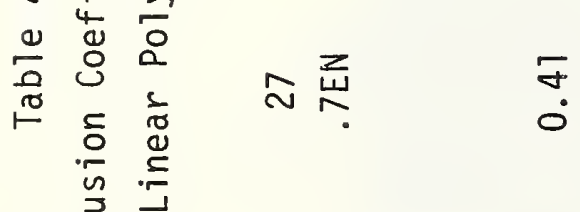

$$
\begin{aligned}
& \text { 妾. }
\end{aligned}
$$

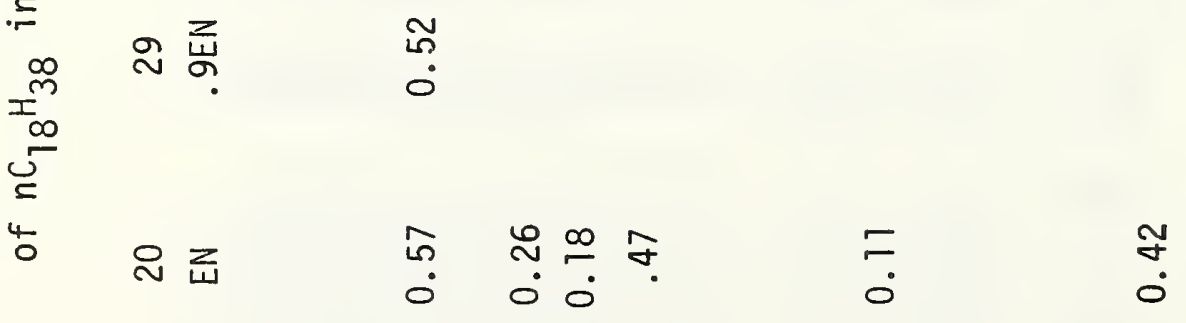

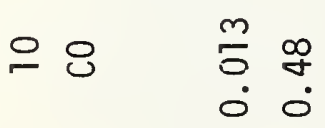

$$
\begin{aligned}
& \stackrel{8}{\square}= \\
& \text { 苛品 }
\end{aligned}
$$

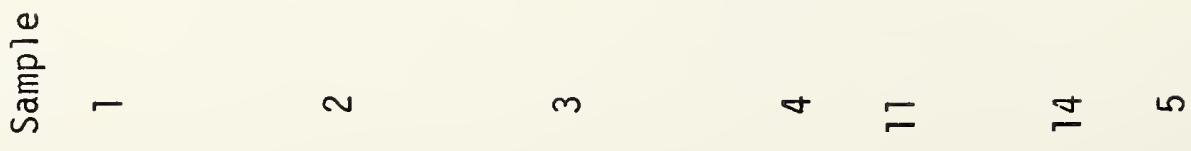


오안웅우

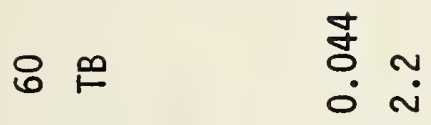

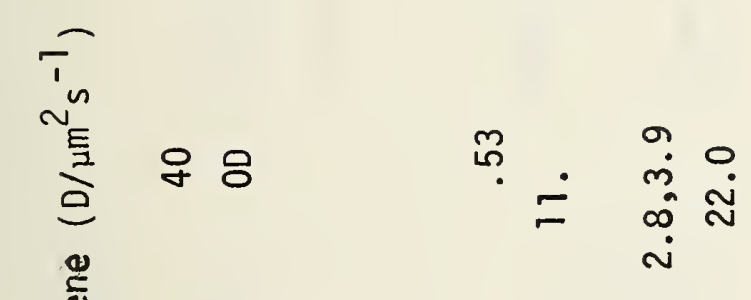

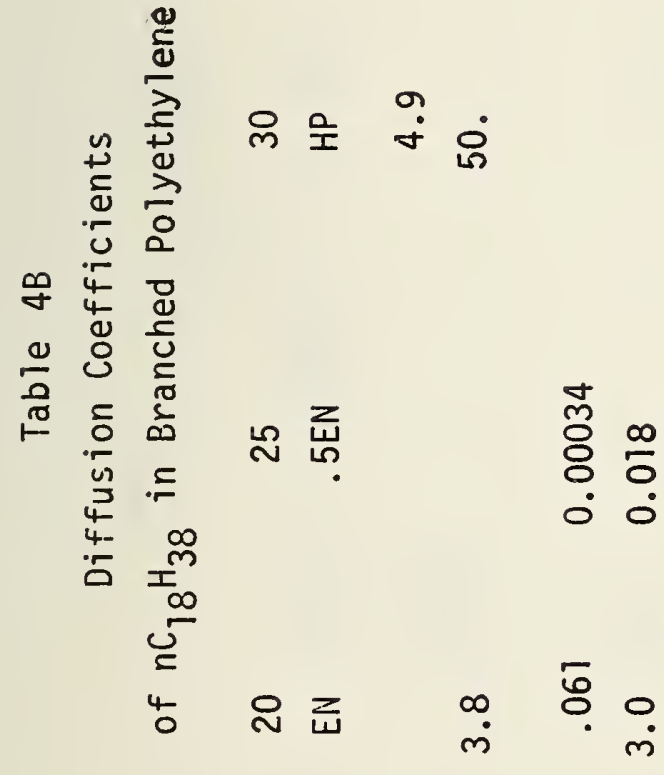

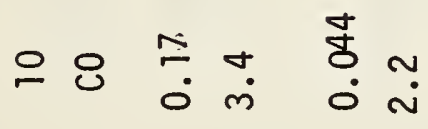

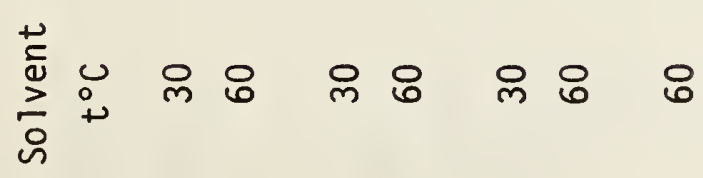

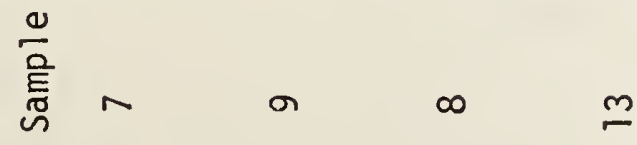


离

3을

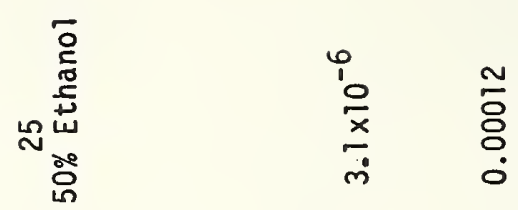

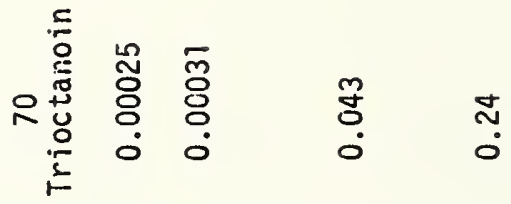

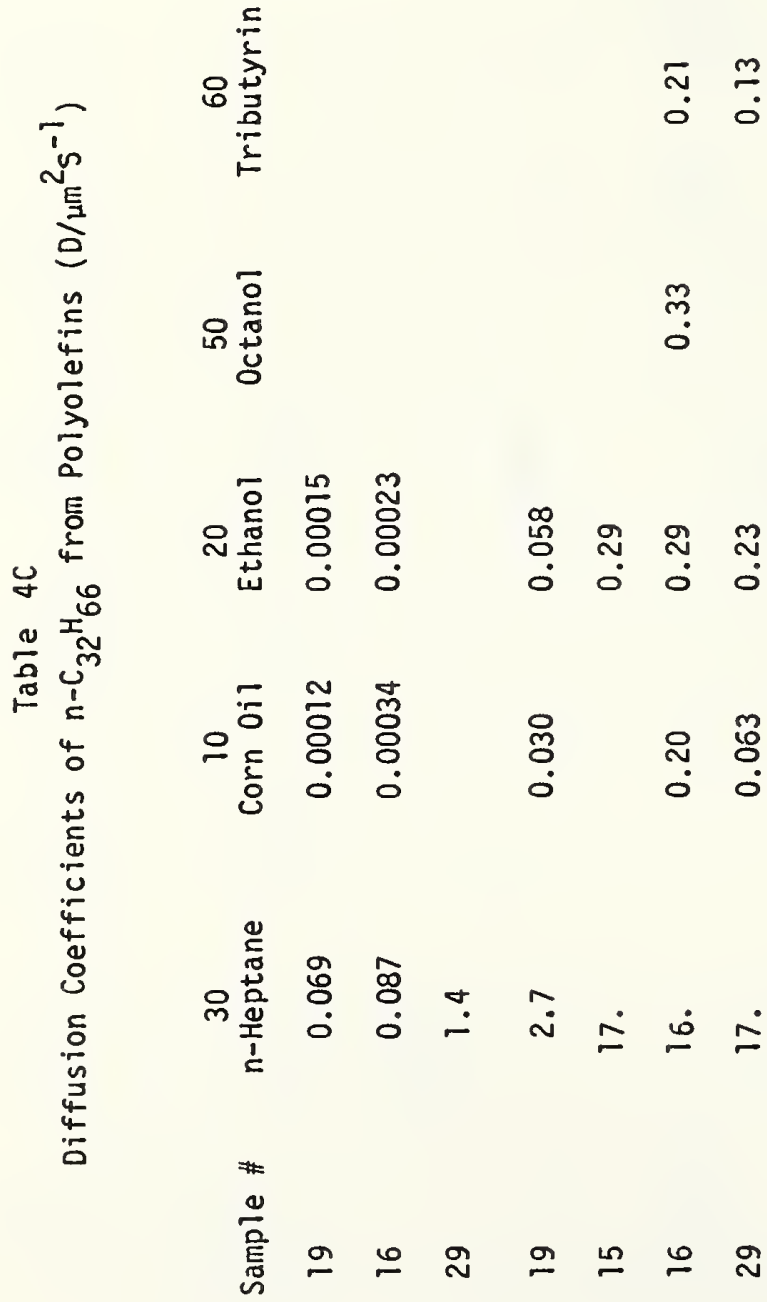

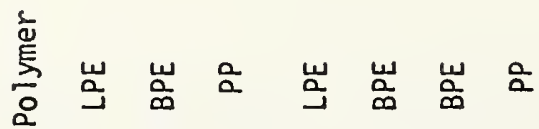

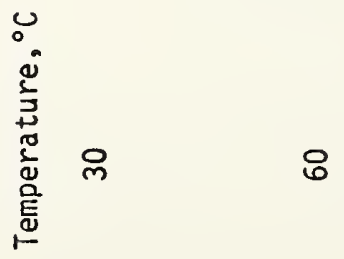




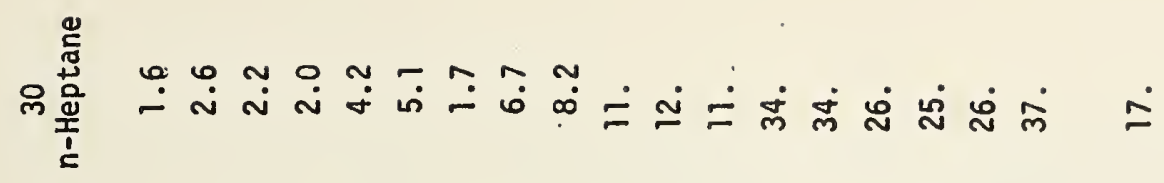

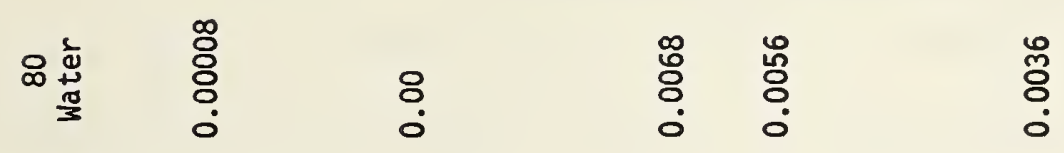

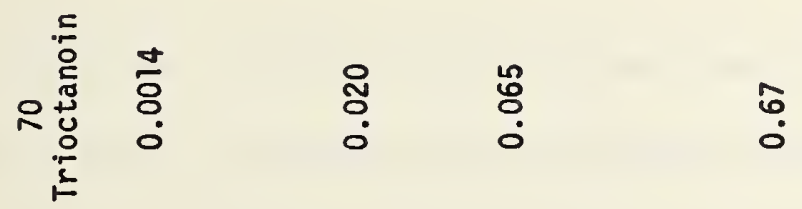

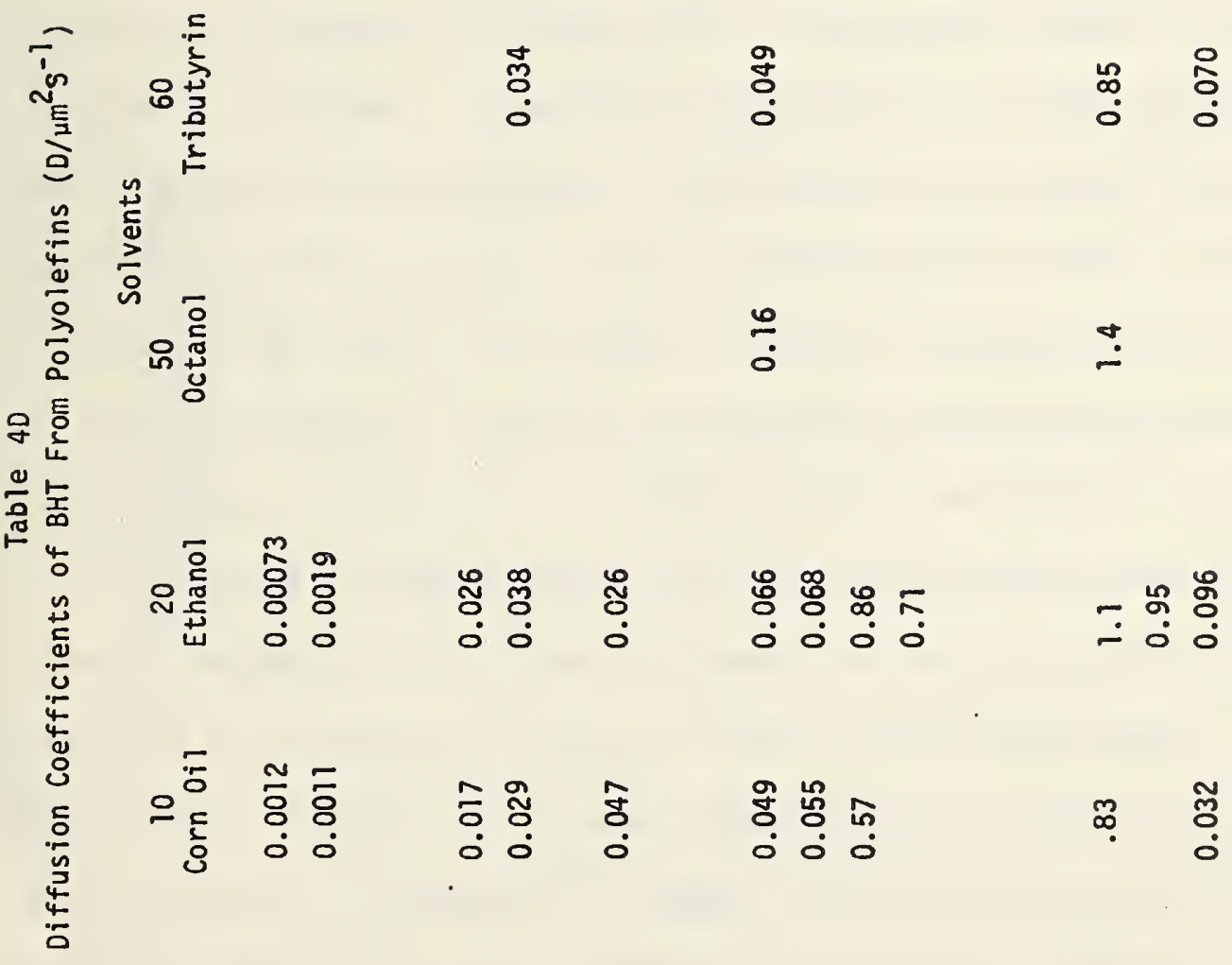

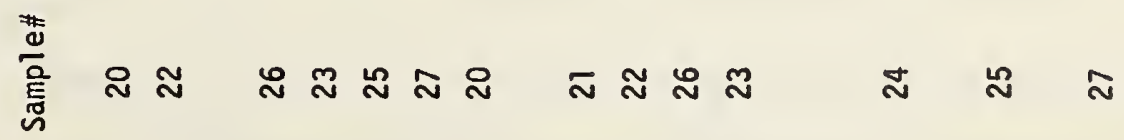

离岕 岕

요요 


\section{Typical Examples of Extraction Experiments}

Typical extraction results may be separated into three categories, namely, ideal Fickian behavior, effect of highly swelling solvents and intermediate cases.

In order to demonstrate the ideal Fickian behavior, in special cases, we may employ the same chemical species as the solvent as well as the migrant, except that the migrant is radioactively labeled. n-octadecane was chosen for these experiments. The polymer was saturated with the migrant/solvent before the extraction experiment. Therefore, change of diffusion coefficient due to swelling or absorption of solvent will not be observed. Under these conditions, the extraction experiments follow an ideal Fickian behavior as shown in Figure $2 A, B$ and $C$ for branched polyethylene saturated with radioactively labeled n-octadecane being extracted by $n$-octadecane at $30^{\circ} \mathrm{C}(840302)$. The curves are calculated as afore mentioned. Both the linear time (Figure $2 \mathrm{~A}$ ) and linear $t^{1 / 2}$ (Figure 2B) representations are commonly used in the literature. The linear $t^{1 / 2}$ representation will show a linear region below 0.6 fractional extraction for partition coefficients favoring the solvent. The log-log representation can also show this 1 inear $t^{1 / 2}$ region as a straight line of slope 0.5 as well as behavior at regions below 0.1 fractional migration.

In a highly swelling solvent, the diffusion coefficient of the migrant will increase drastically as the polymer matrix expands. A typical example is shown as Figure $3 A$ and $B$ for the extraction of $n$ dotriacotane from polypropylene by $n$-heptane at $60^{\circ} \mathrm{C}(2930601)$. The diffusion coefficient changes at minimum by a factor of more than 10 . The maximum diffusion coefficient observed in this case at high degree of extraction is only the lower bound of the diffusion 


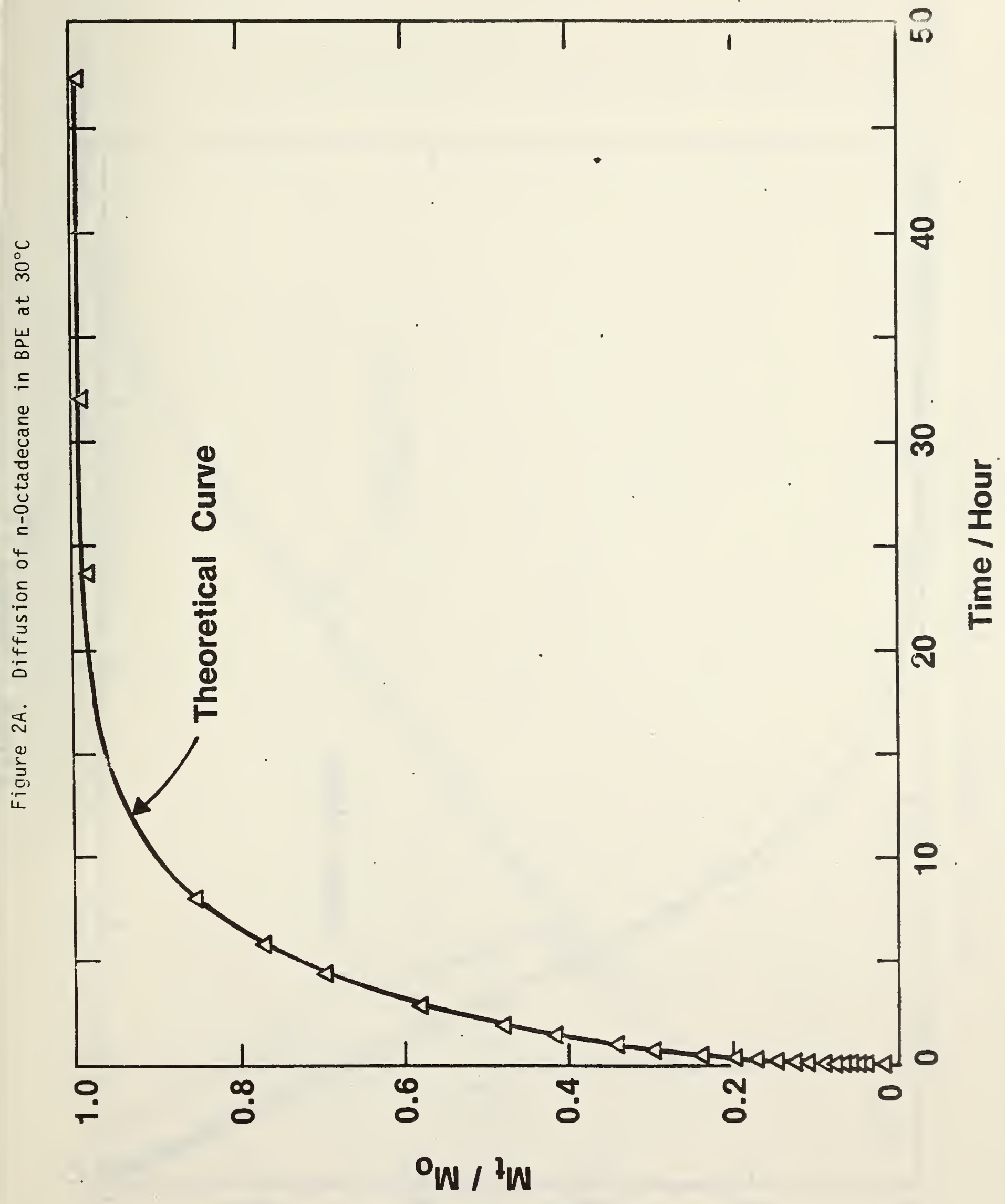




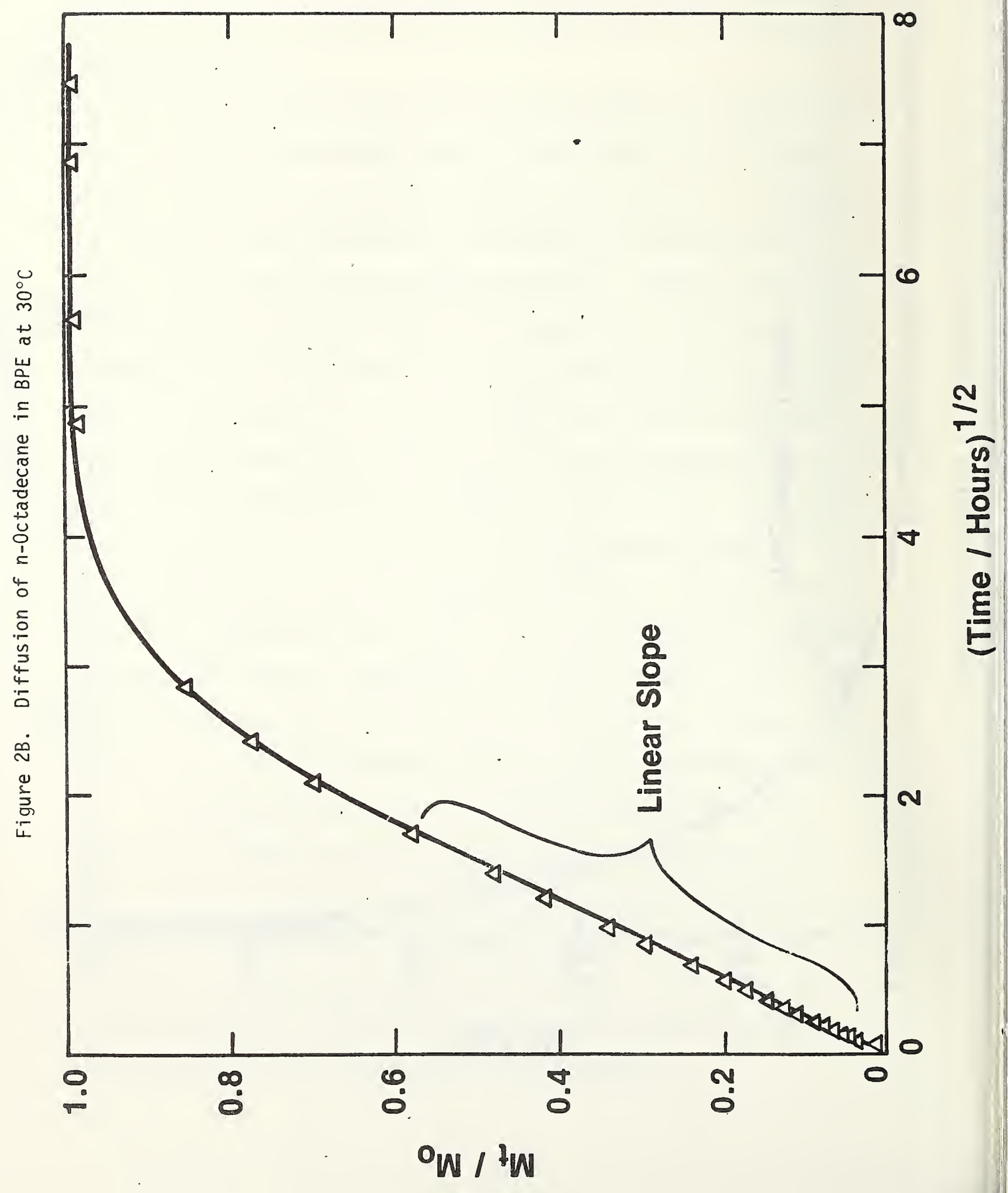




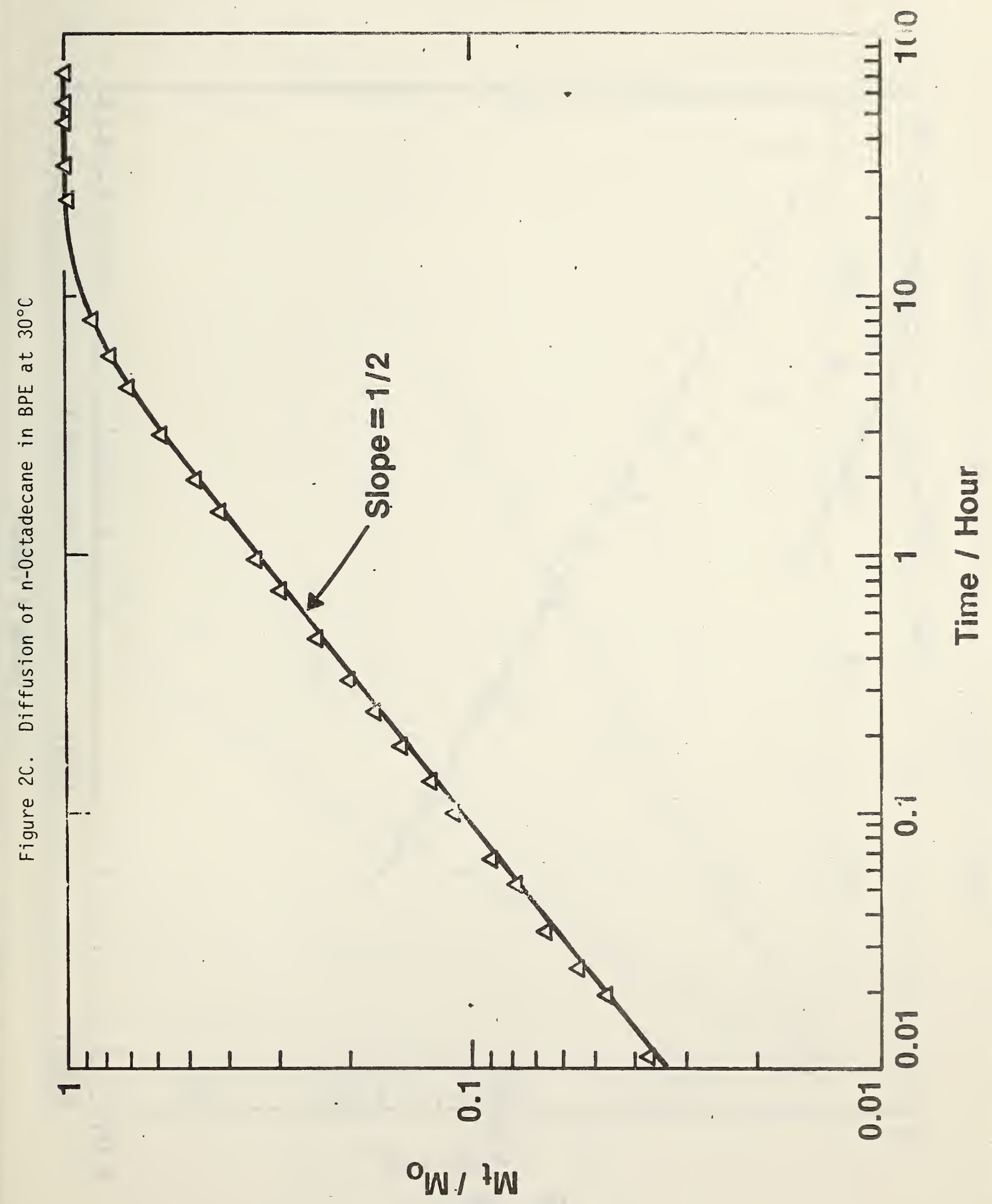




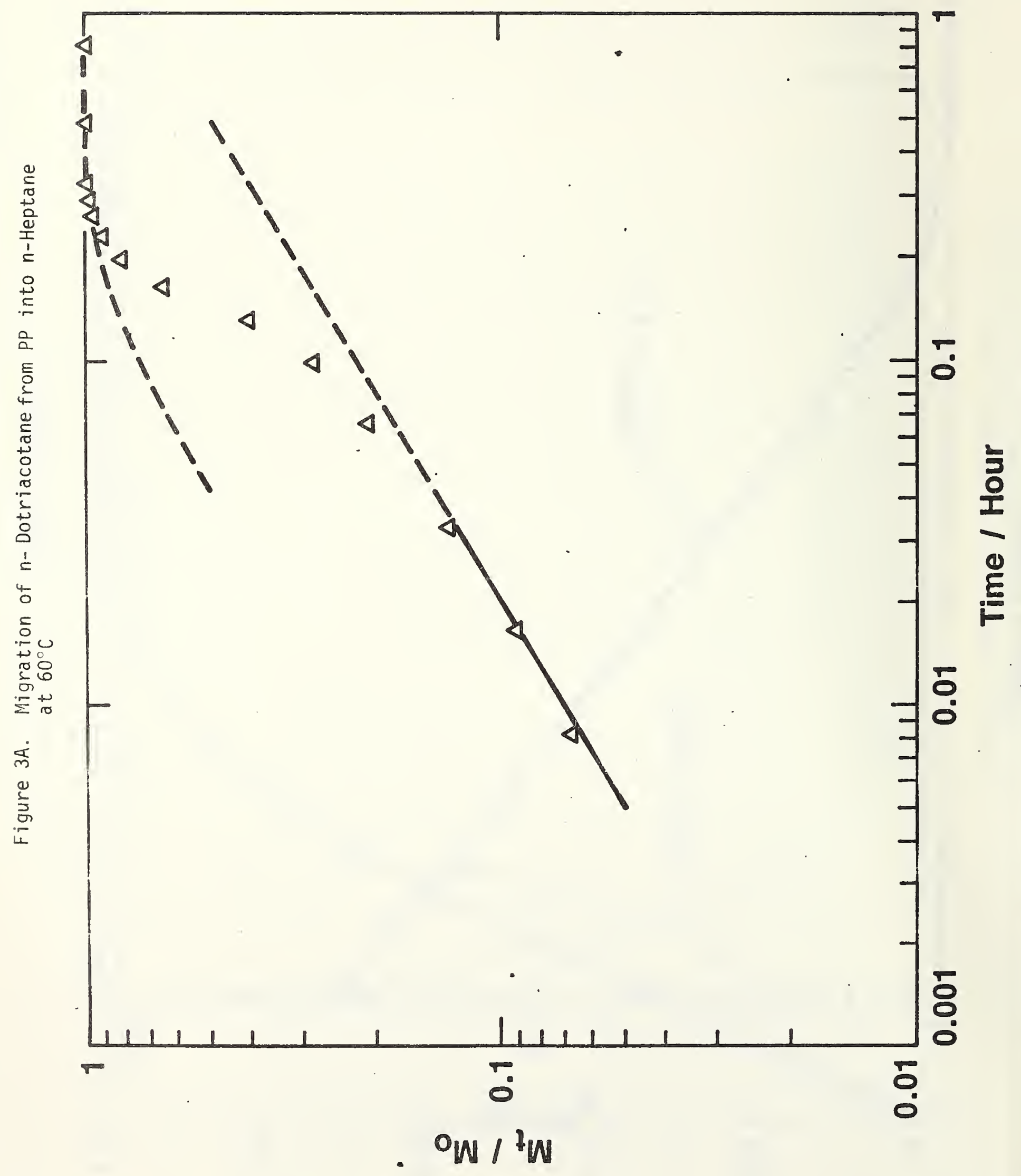




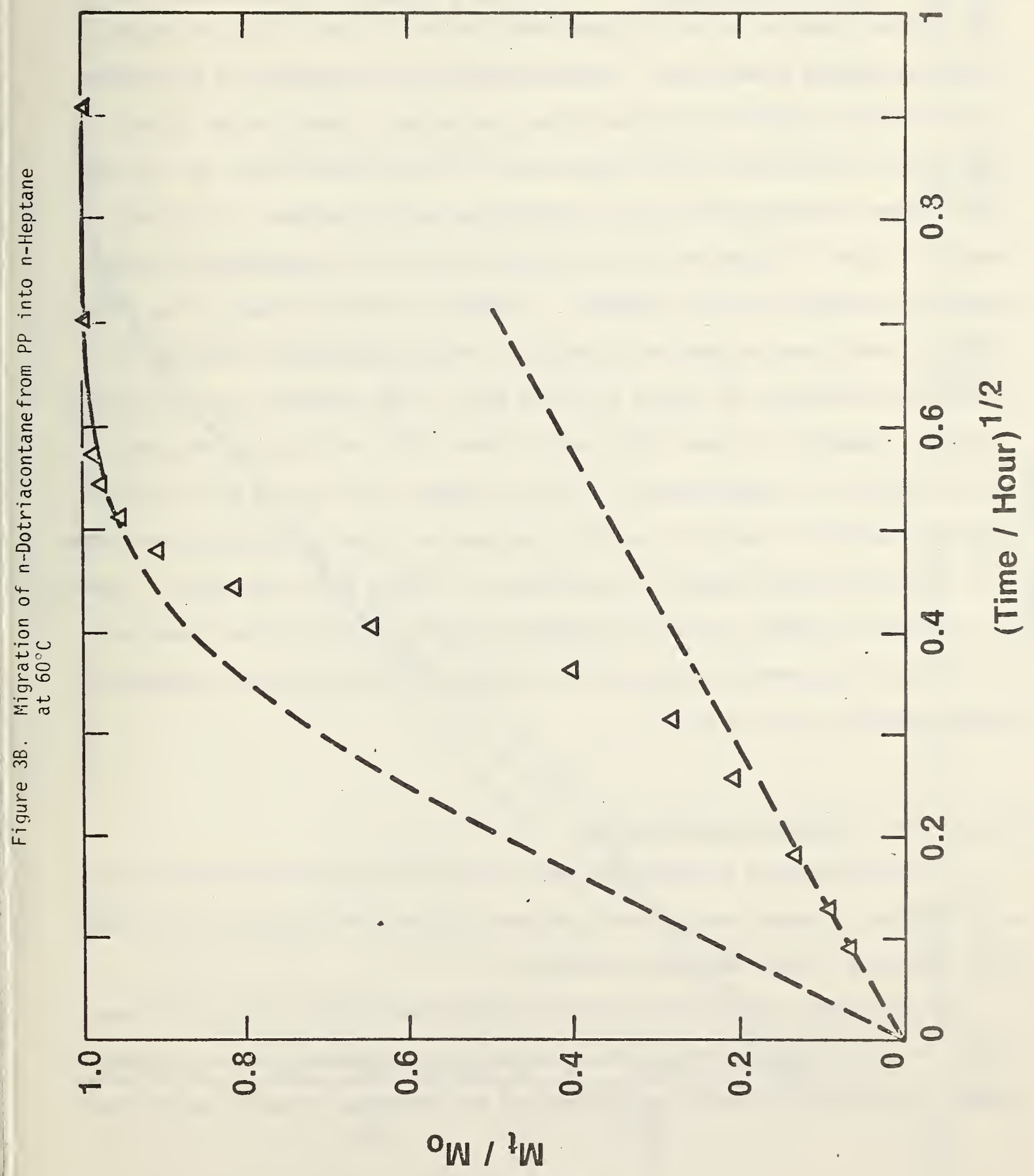


coefficient under equilibrium swelling, as most of the migrant have already been extracted by the time the sample reaches equilibrium swelling.

For most cases of extraction experiments the results would fall in between of these two examples shown above. A moderate swelling or absorption of solvent may occur before the majority of migrant being leached out. Therefore the typical behavior will show up as a shift between two diffusion coefficients, one for the "dry" polymer and the other for the "equilibrium swollen" polymer. A typical example is shown in Figure $4 A$ and $B$ for the extraction of $n$-octadecane from polypropylene by ethanol at $30^{\circ} \mathrm{C}(2820300)$. In general either the lower or the higher diffusion coefficient regions are expanded or reduced depending on the rate of solvent equilibration. One should also note that if the migration curve is shown in Figure $4 B$, commonly as linear Mt/Mo versus linear $t^{1 / 2}$, one may draw the conclusion that the behavior is rather ideally Fickian. However, the log-log plot in Figure $3 \mathrm{~A}$ indicates detail behavior below $10 \%$ of extraction. The 1og-log plot does suffer from resolution at high degree of extraction, i.e. above $70 \%$ of extraction. However, for regulatory purposes, the detailed behavior in this region is less important. As with such high degree of extraction, one may simply use $M_{0}$ as the extractables without producing serious errors.

\section{Correlation of Diffusion Coefficients}

In the following we present empirical correlations of diffusion coefficients as a function of temperature, solvent, migrant, migrant concentration, or polymer, while keeping all other parameters constant.

Due to the wide span of the diffusion coefficients $\left(10^{8}\right)$, log-log plots are used. The solid diagonal line in the correlation plot denotes a 1:1 correspondence between the subject diffusion coefficient and the reference diffusion coefficient. 


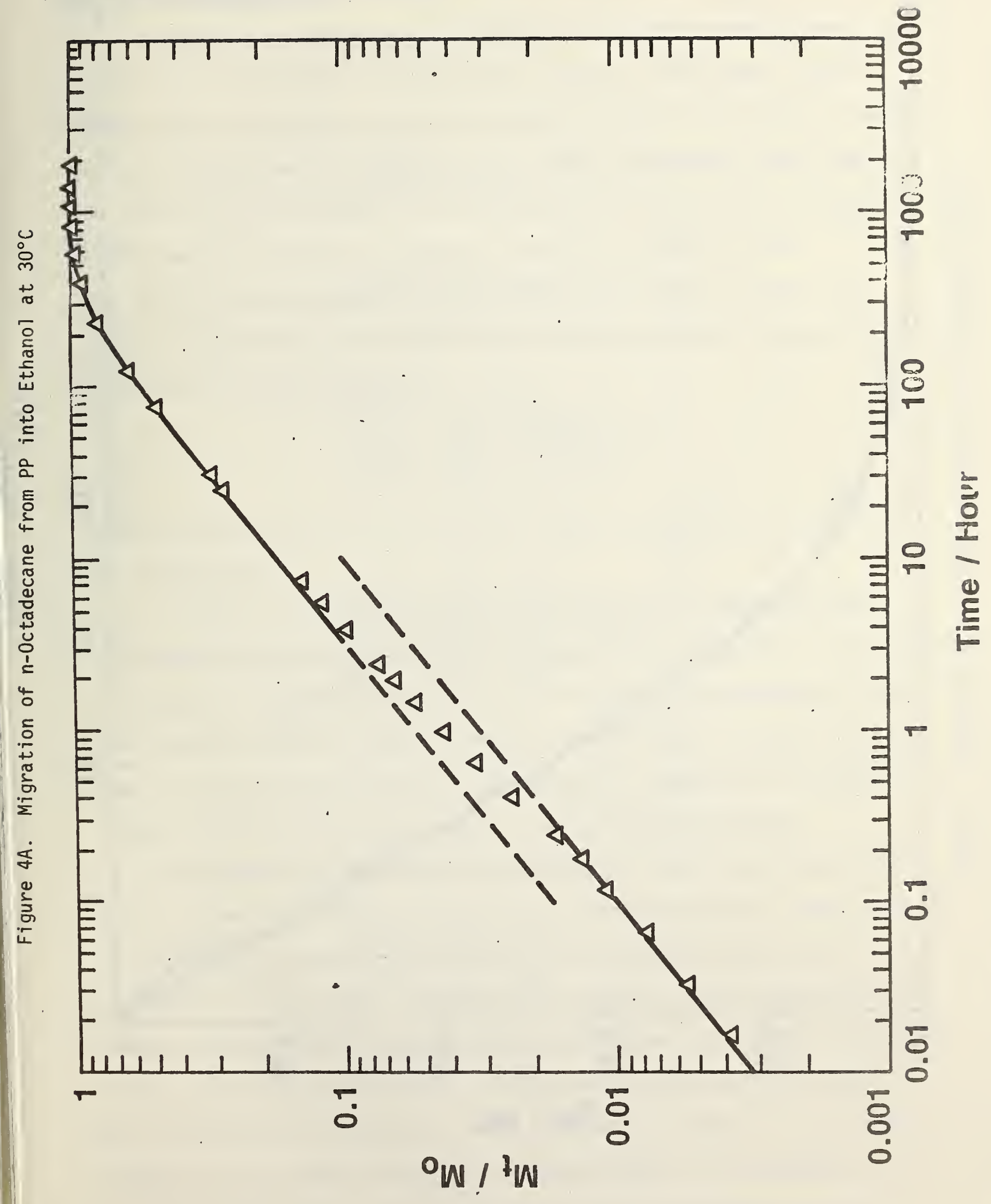




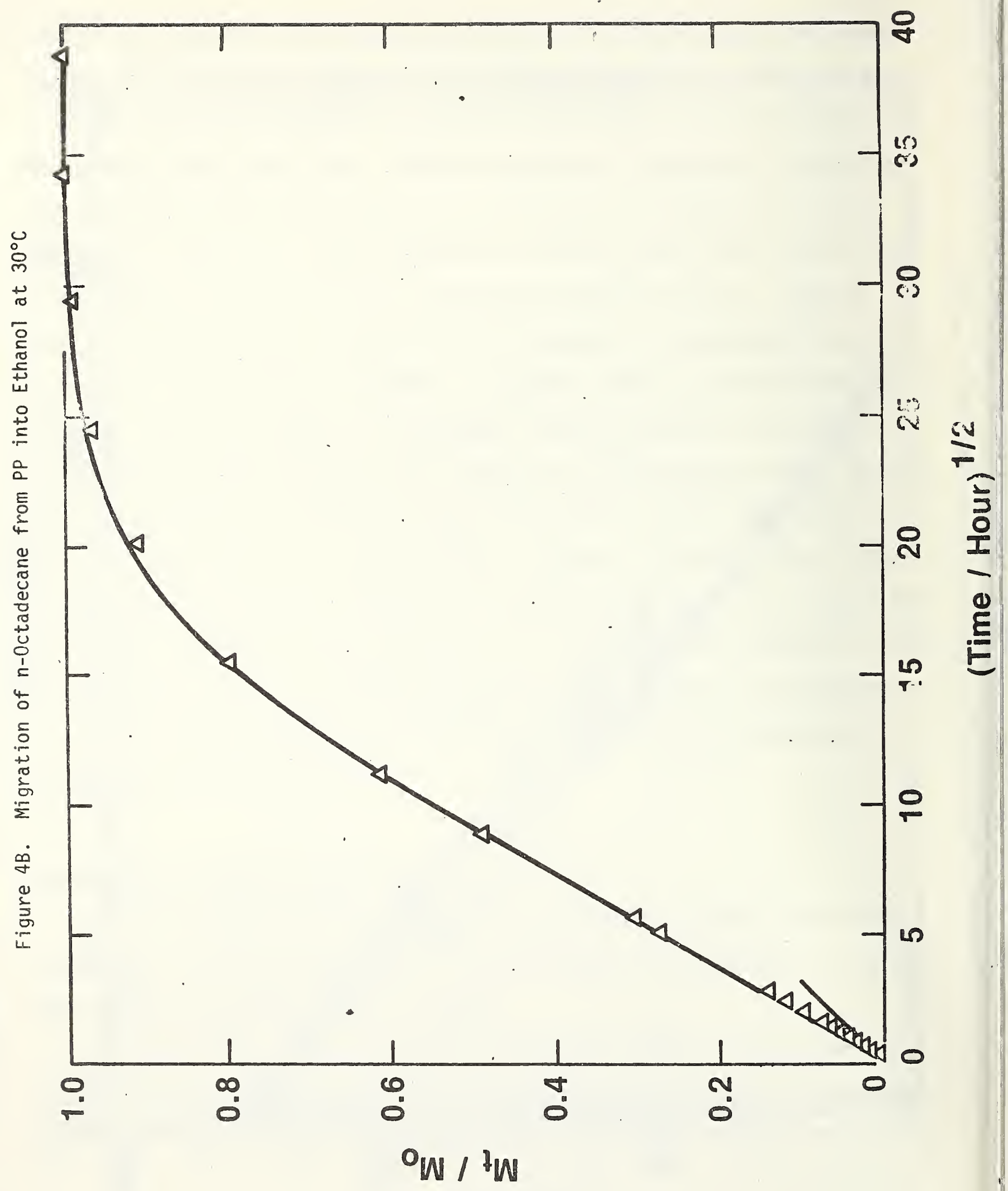




\section{Effect of Temperature}

Temperature will have pronounced effect on solvent extractibilities not only through the changes in solubilities, partition coefficients, swelling powers but also through the possible change of states of the polymer, migrant as well as the solvents. However in the limited temperature ranges that is of interest to food packaging, for instances from -10 to $100^{\circ} \mathrm{C}$, the change in the diffusion coefficient is generally less than a factor of 1000 .

For the limited temperature range baring any change of states, the effect of temperature may be best described by the activation energy defined by $E$ in the following expression:

$$
D_{2} / D_{1}=\exp -\frac{E}{R}\left(\frac{1}{T_{2}}-\frac{1}{T_{1}}\right)
$$

where $D_{2}$ and $D_{1}$ are the diffusion coefficients at temperatures $T_{2}$ and $T_{1}$ (in K), respectively.

Estimation of activation energies are tabulated in Table 5A through $F$ and presented graphically in Figure $5 A$ through $F$ for n-octadecane in triglycerides, in ethanol and ethanol/water mixtures, in n-heptane, in n-octadecane, for $n$ dotriocotane and BHT in various solvents, respectively. A straight-line is used to link the two data points at 30 and $60^{\circ} \mathrm{C}$. A majority of the results listed in Table 3 and 4 are presented in these tables and figures.

The groupings of the diffusion coefficient in each of the figures are due to polymers or solvents. In general, the behavior of PP is rather similar to that of BPE, although the magnitudes of the diffusion coefficients in PP are closer to that in LPE. The behaviors in ethanol, pure triglycerides and corn oil are very similar to each other in magnitudes of activation energies as well as diffusion coefficients. In Figure 5G, diffusion coefficients of BHT in both corn oil and water as measured by NBS for Authur D. Little (ADL) samples as well as data from ADL are plotted together. The agreements in corn oil are excellent. The measurements in water are probably within a factor of 3 from mean values. 


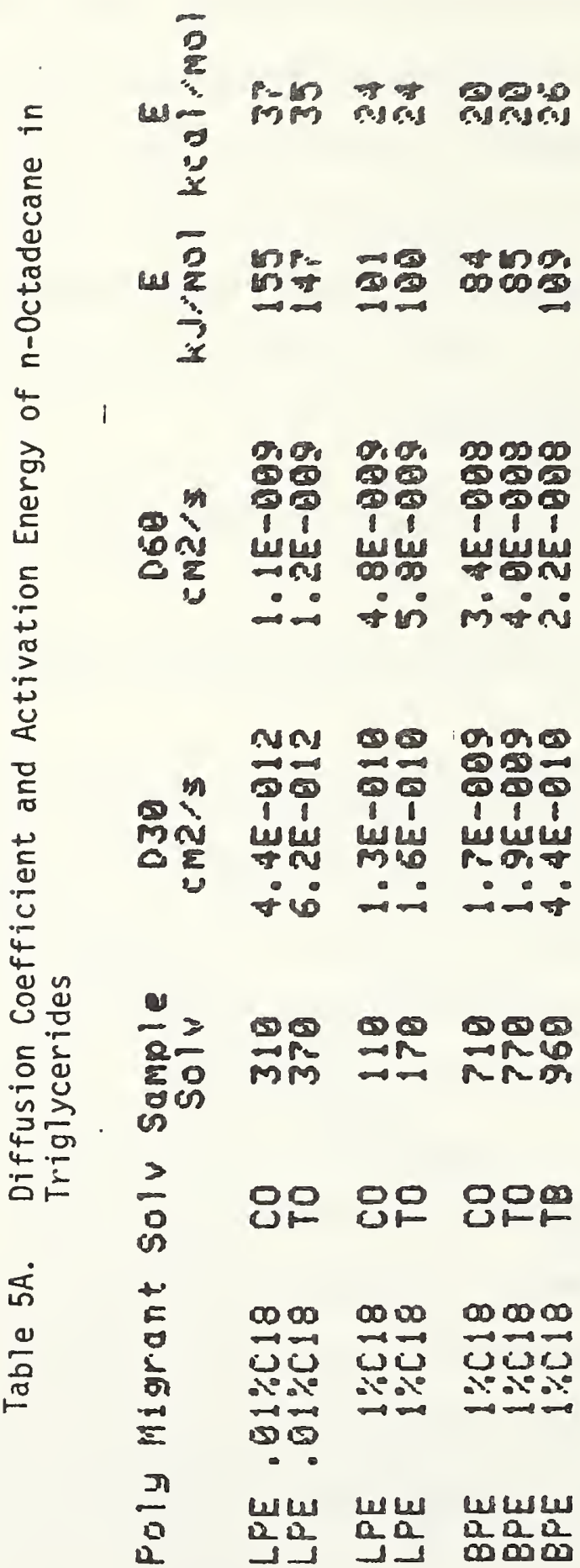




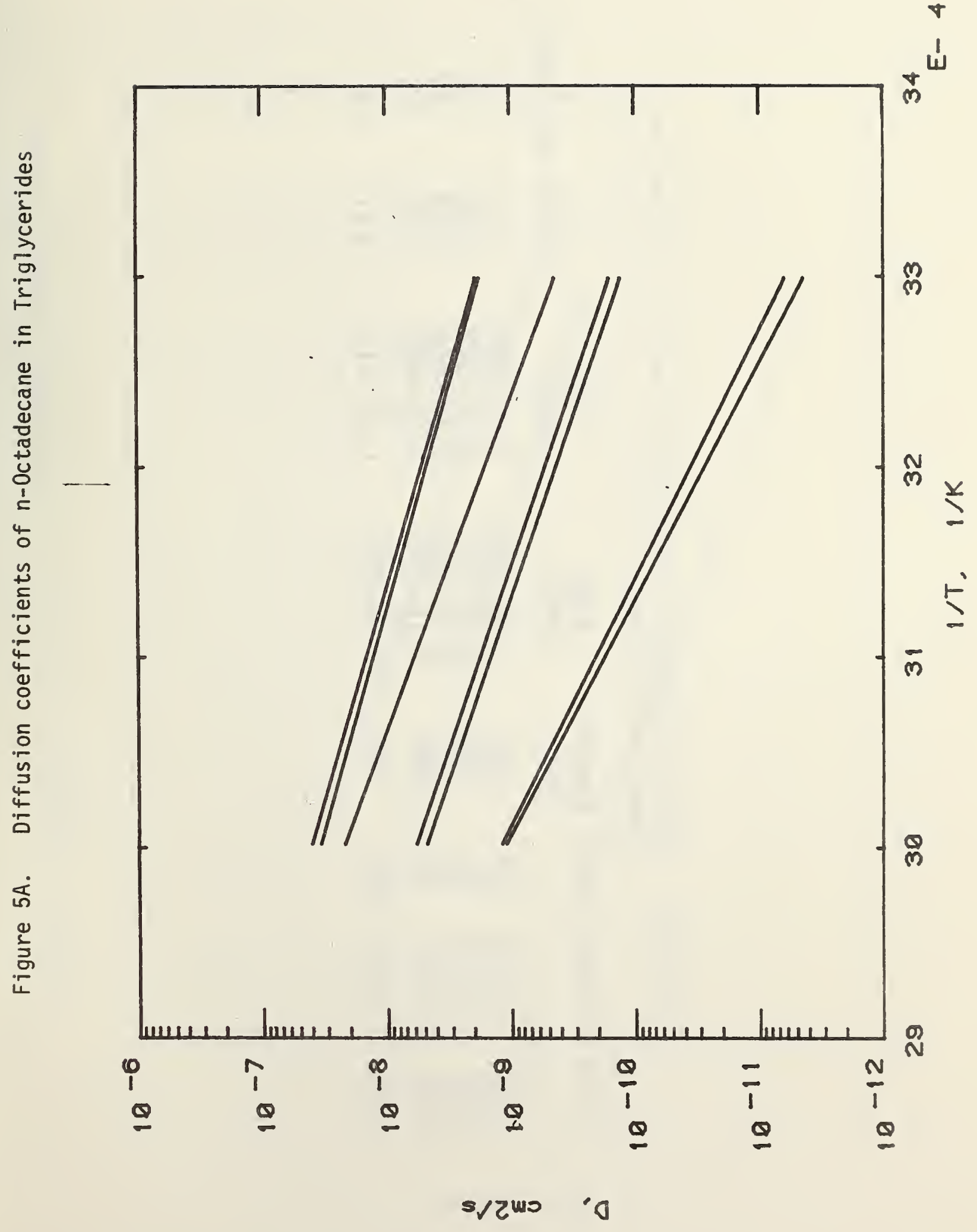




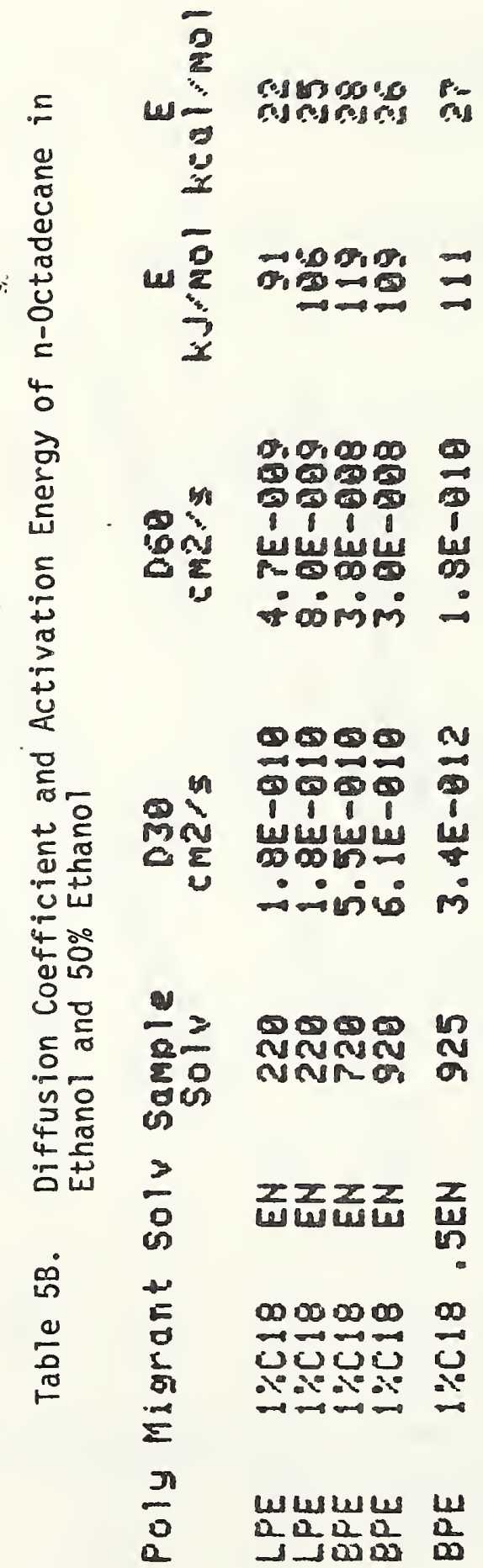




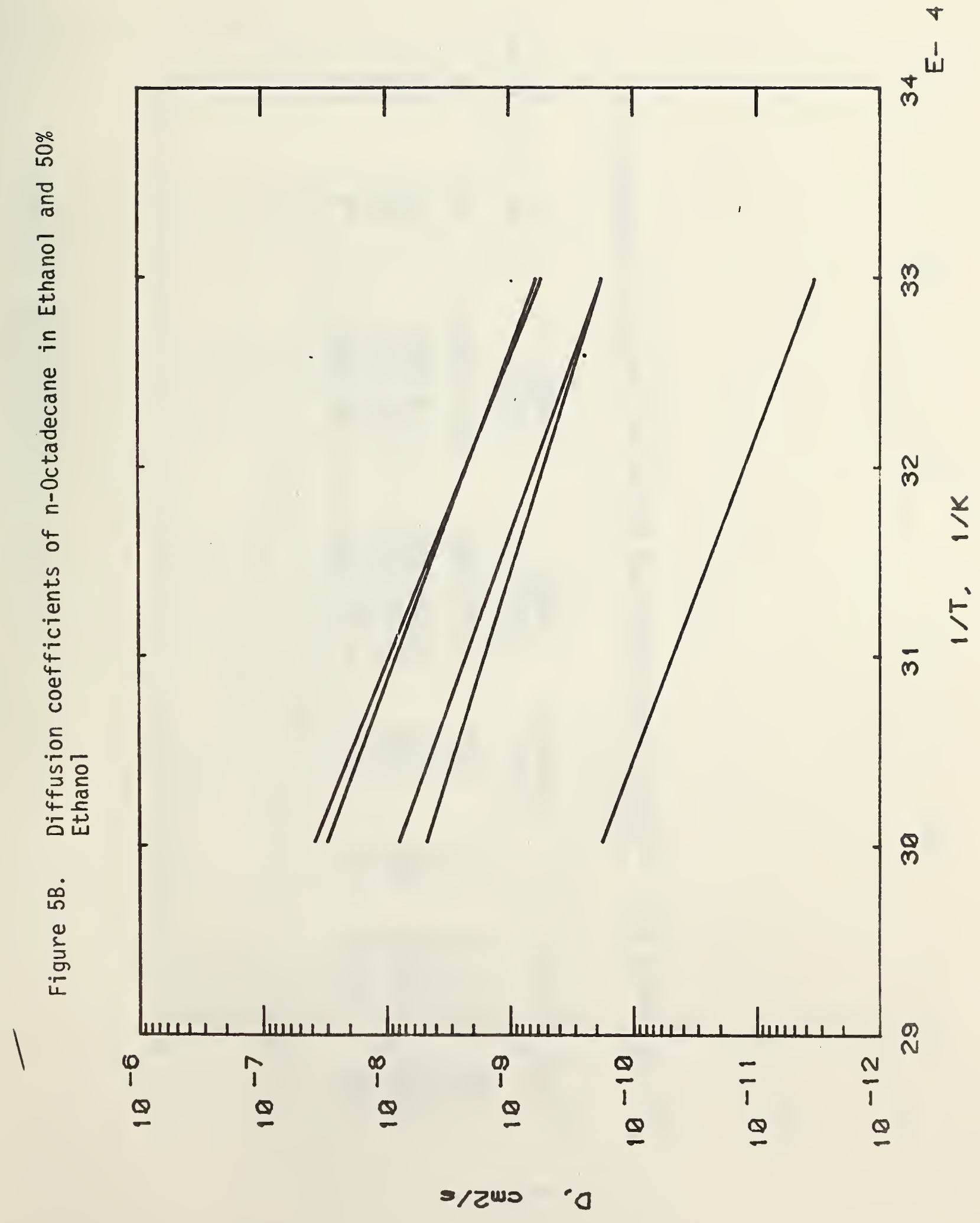




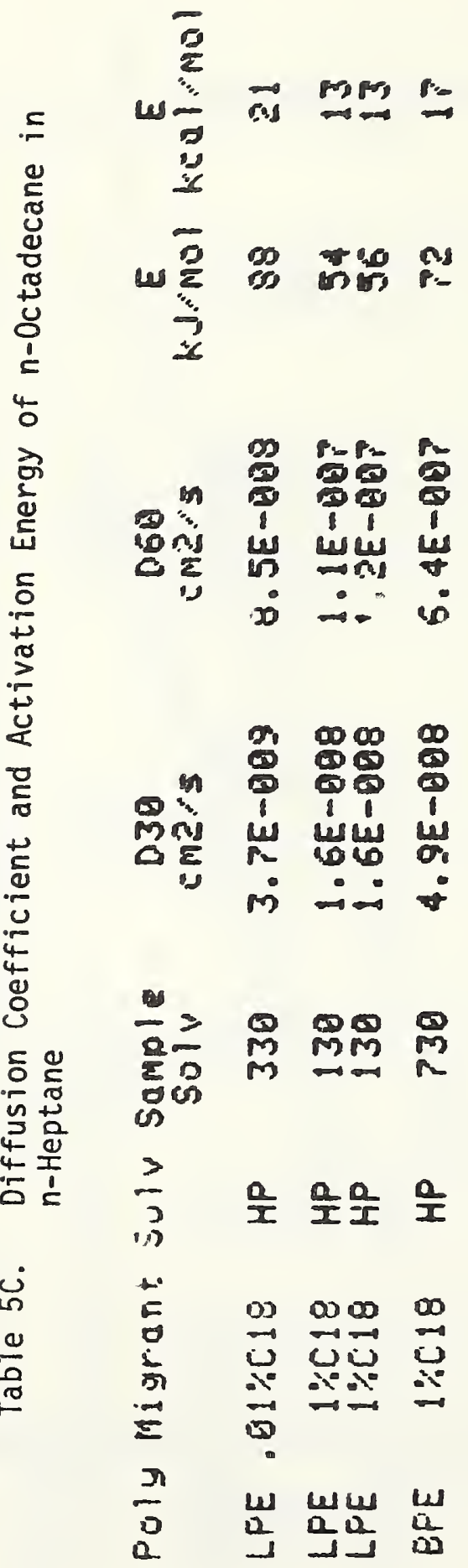




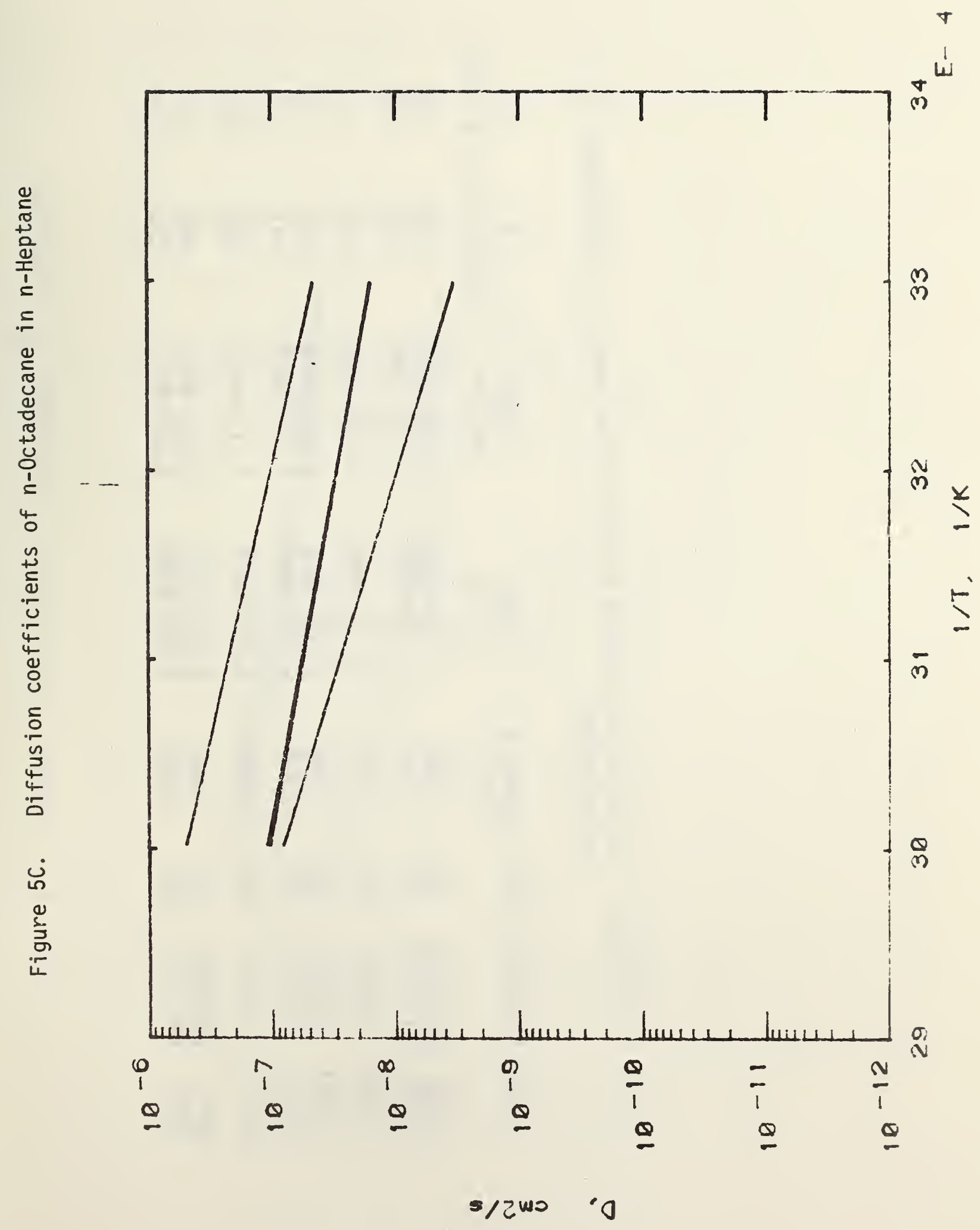




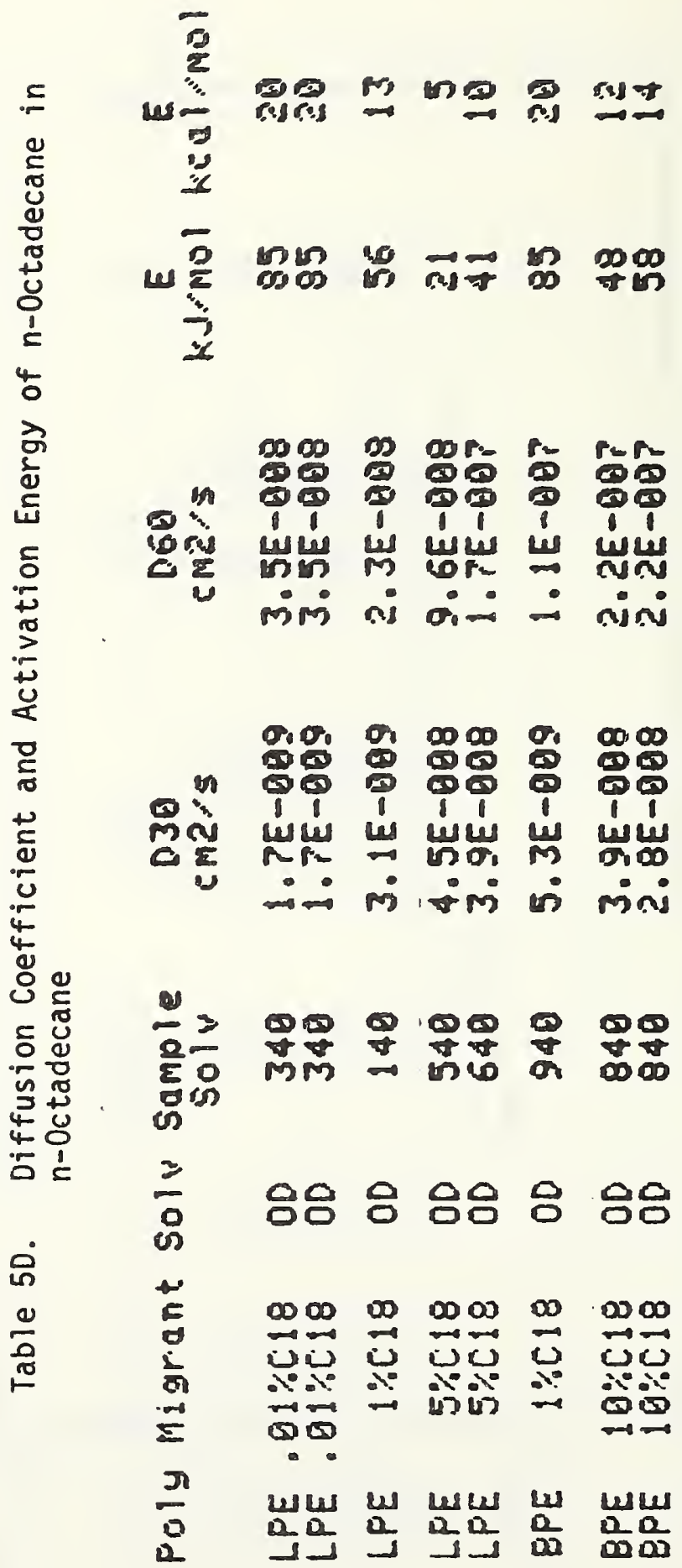




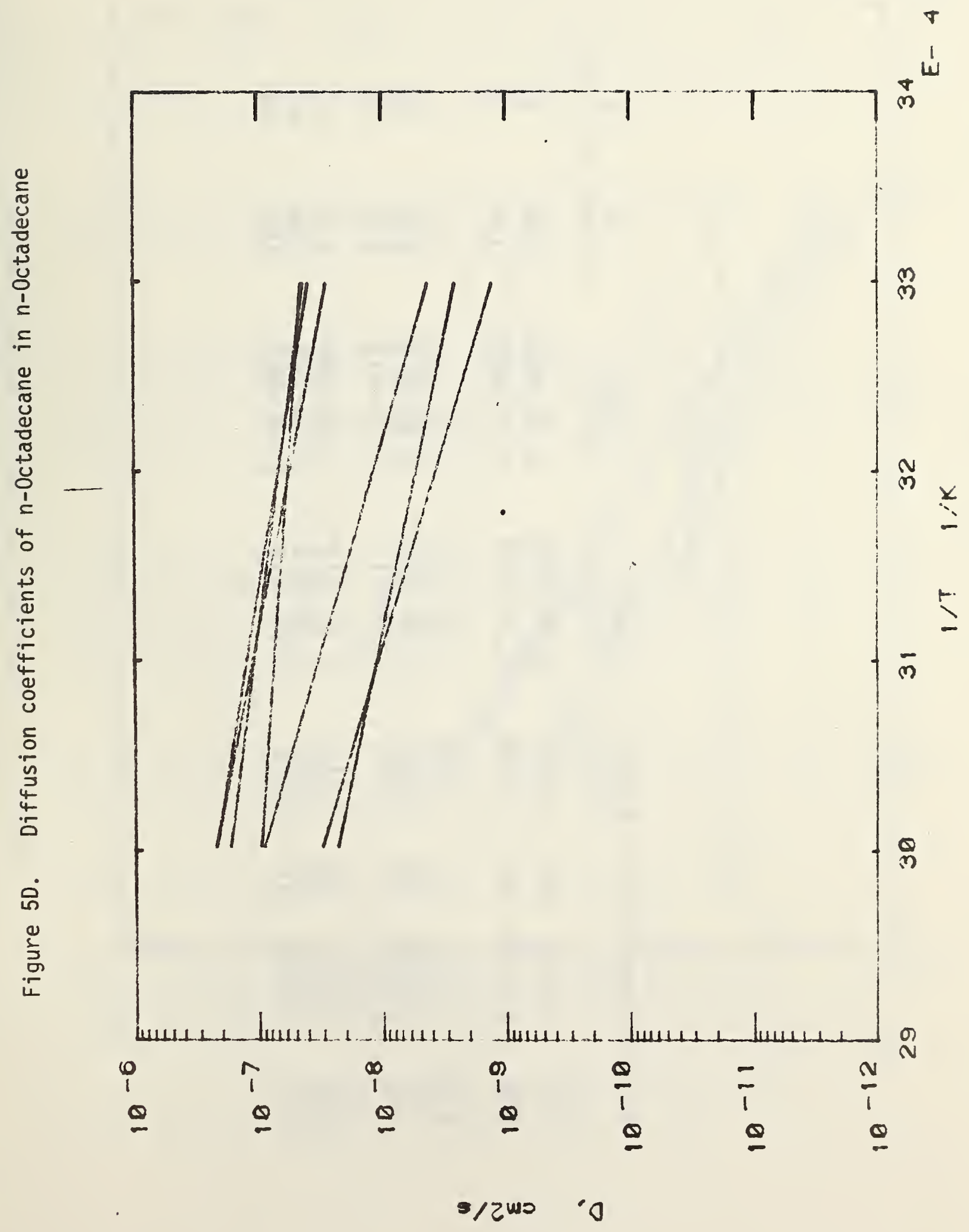




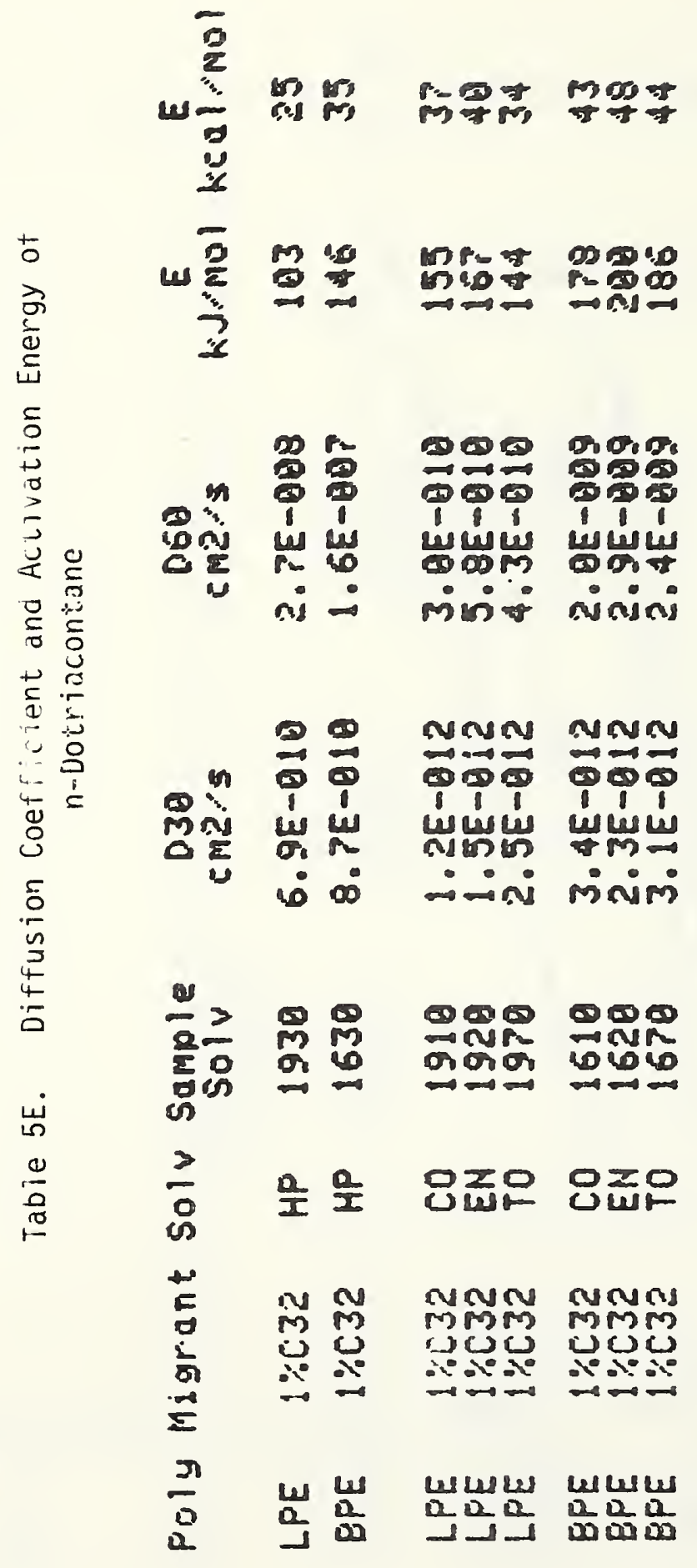




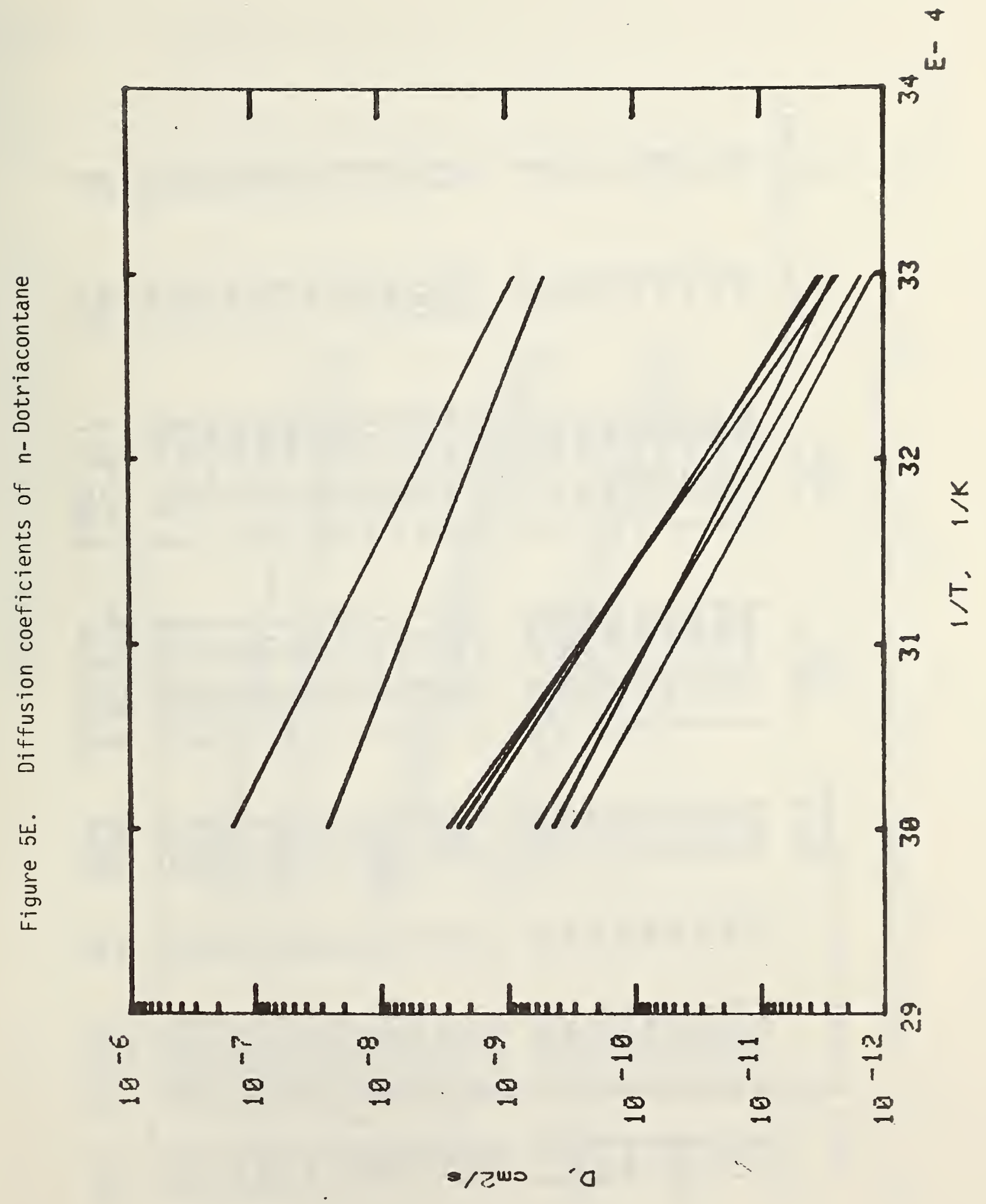




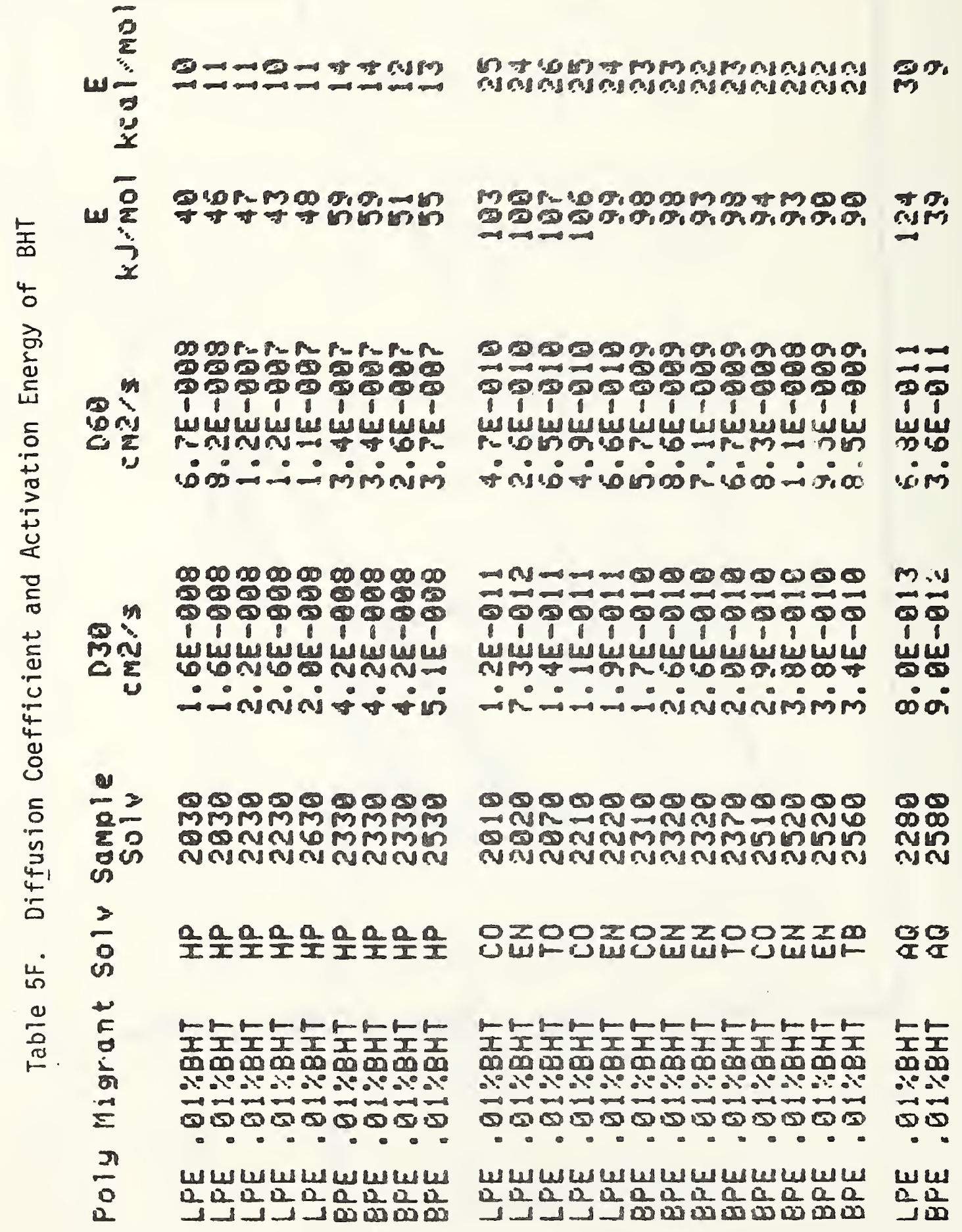




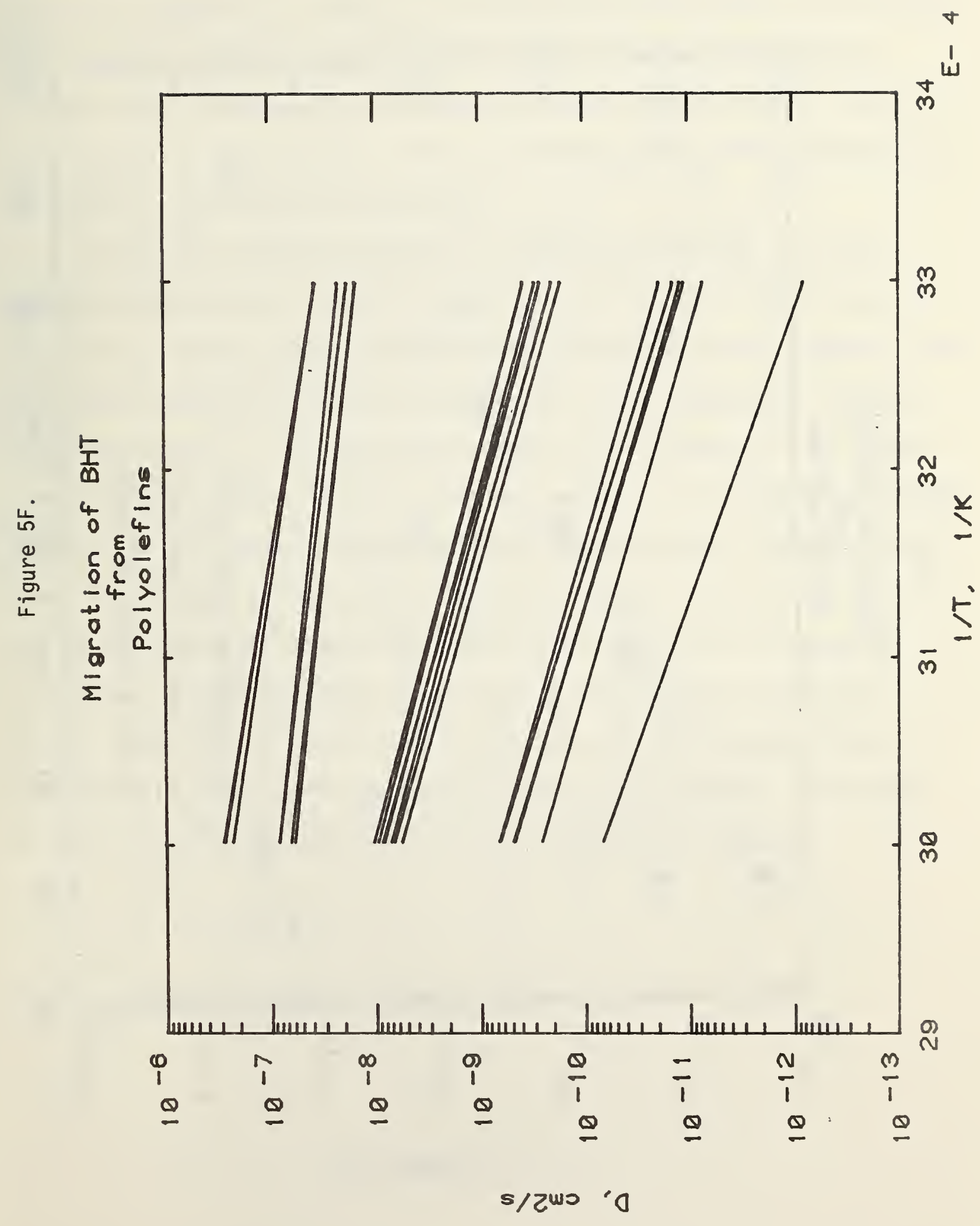




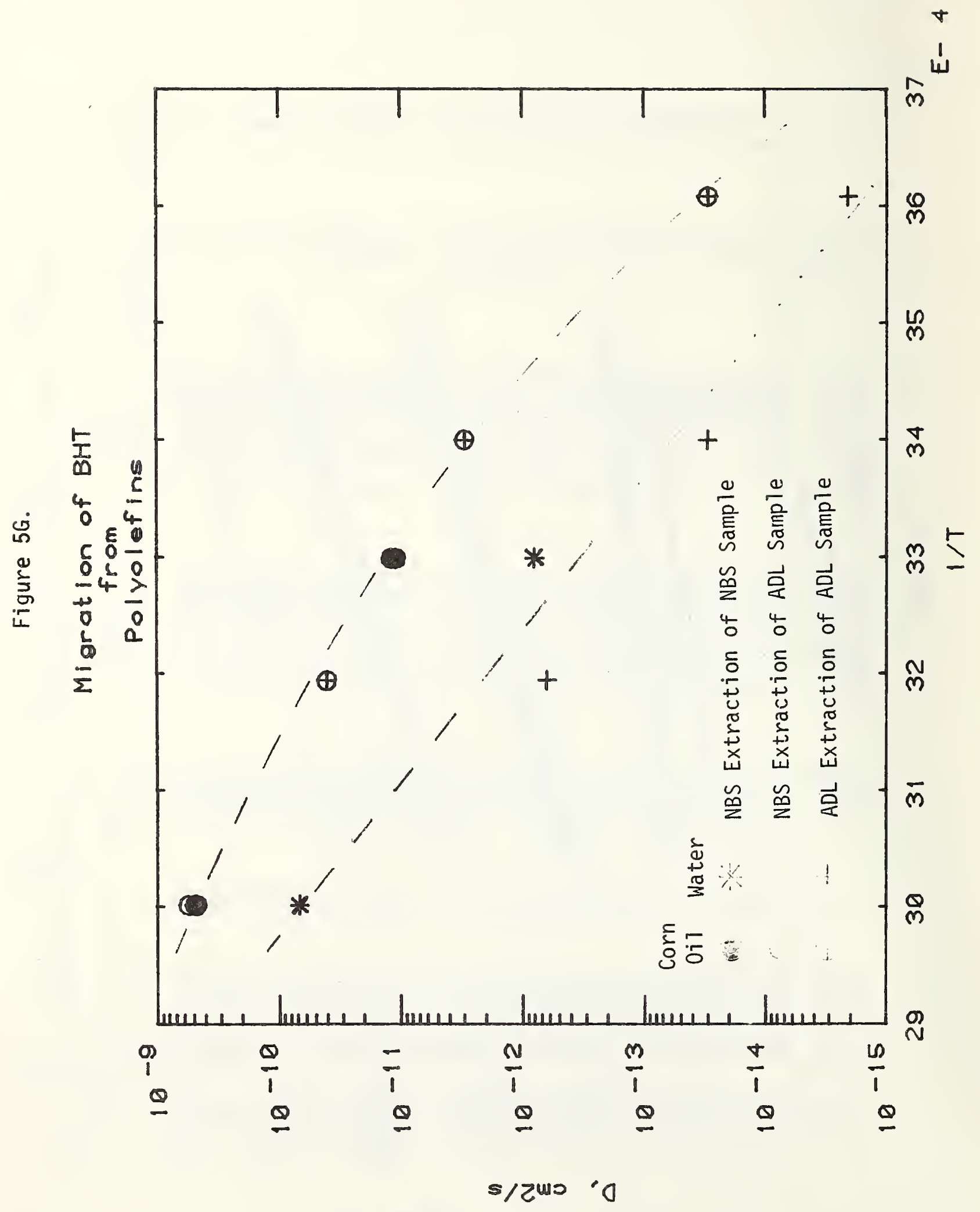


The diffusion coefficients at $30^{\circ} \mathrm{C}$ are less than that of at $60^{\circ} \mathrm{C}$ as expected as shown in Figure 6 . There is a general trend that the effect of change of temperature is greater at lower diffusion coefficient. By grouping into migrants, empirical correlation may yield a scatter of less than a factor of 5. n-Octadecane and BHT may be grouped together without effecting the correlation significantly. Better correlation for $n$-dotriacontane may be obtained by separating into LPE and BPE.

The average activation energies for various combinations of polymer, migrant and solvent are listed in Table $6 A, B$ and $C$. No estimated range is given for activation energies obtained from a single pair of estimation. The activation energies or ratios of $\left(D_{60} / D_{30}\right)$ for $n$-dotriacontane and BHT are plotted in Figure 7 . The activation energies for n-octadecane show similar trend but with a higher scatter of the data points. It is somewhat surprised to find the four combinations having linear correlations of identical slope. These correlations indicate that although the changes in activation energies due to changes in the reference diffusion coefficients are the same, the activation energies are higher in the case of BPE than that of LPE, and higher in the case of $n$-dotriacontane than that in n-octadecane. These correlations are not to be extrapolated toward high diffusion coefficients, as such extrapolations may soon lead to a situation that $D_{30}>D_{60}$. 


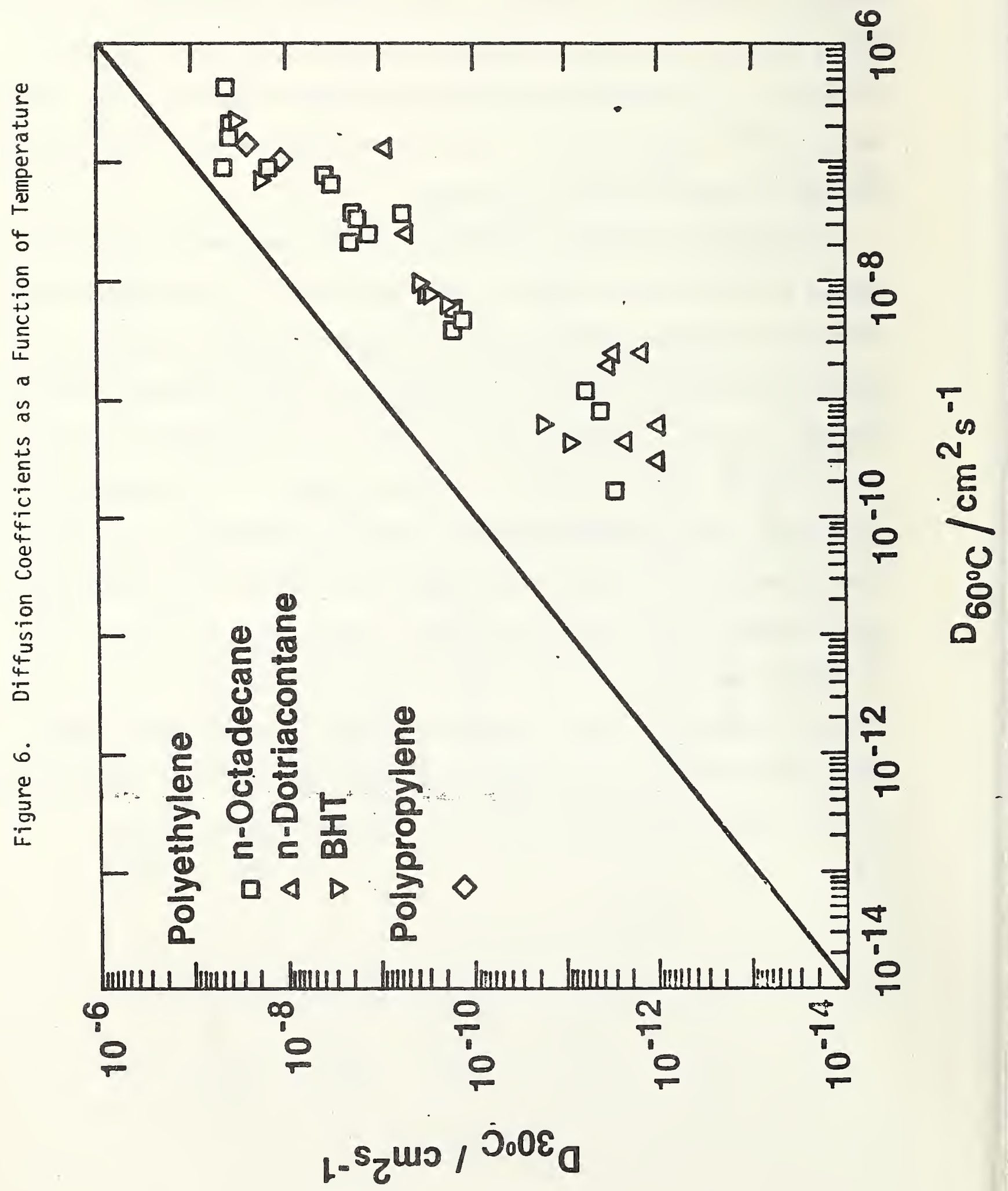




\section{Table $6 \mathrm{~A}$}

Activation Eneraies of Migration of $n$-Octadecane at Different rencentrations

( $E, \mathrm{~kJ} \mathrm{~mol}^{-1}$ )

\begin{tabular}{|c|c|c|c|c|c|}
\hline into from & $0.01 \%$ & $\begin{array}{l}\text { LPE } \\
1 \%\end{array}$ & $5 \%$ & $1 \%$ & $10 \%$ \\
\hline n-Heptane & 88 & 55 & & 72 & \\
\hline$n$-0ctadecane & 85 & 56 & $30+10$ & 85 & $53 \pm 5$ \\
\hline $\begin{array}{l}\text { Ethanol, } \\
\text { Triglycerides }\end{array}$ & $151+4$ & $\begin{array}{r}99+ \\
100 \pm\end{array}$ & & $\begin{array}{c}114+5 \\
93 \mp 15\end{array}$ & \\
\hline $50 \%$ Ethanol & & & & 111 & \\
\hline
\end{tabular}

Table 6B

Activation Energies of Migration of $n$-Dotriacontane

$\left(E, \mathrm{~kJ} \mathrm{~mol}^{-1}\right)$

\begin{tabular}{l|ll}
\hline into from & LPE & BPE \\
\hline n-Heptane & 103 & 146 \\
Ethanol, Triglycerides & $155 \pm 12$ & $188 \pm 11$
\end{tabular}

Table 6C

Activation Energies of Migration of BHT

( $E, \mathrm{KJ} \mathrm{mol}^{-1}$ )

\begin{tabular}{l|cl}
\hline into from & LPE & BPE \\
\hline n-Heptane & $45 \pm 3$ & $56 \pm 4$ \\
Ethanol, Triglycerides & $103+4$ & $94 \pm 3$ \\
Water & 124 & 39
\end{tabular}




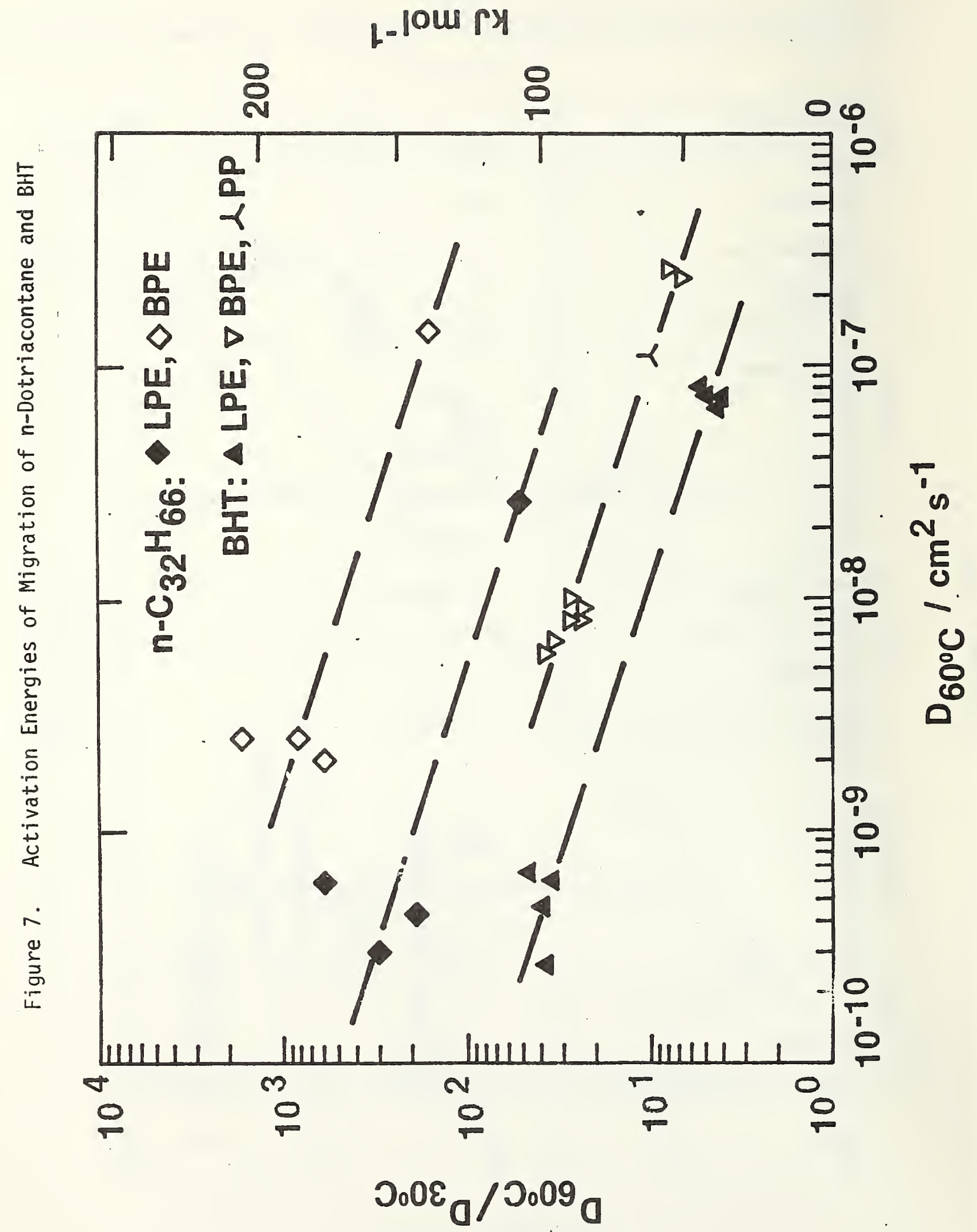


Effect of Solvent

Solvent is the single most important parameter effecting both the amount extractable and the diffusion coefficient of the migrant through solubilities and its swelling action upon the polymer.

By comparing diffusion coefficients in other solvents to one of solvent, e.q. ethanol, while keeping all other parameters constant, it is possible to reduce the large range of $10^{8}$. in the diffusion coefficient for individual solvent to a factor of 5 or less from the correlation.

For partitioning or lower solubility solvents, the correlations may depend not only on the solvent but also on the migrant or polymer. However for solvents having moderate solubilities toward the migrants, the correlation seems to be independent of migrant, polymer or temperature.

The most significant correlations are that for pure triglycerides and ethanol as food oil simulants and that for accelerating solvent, $n$-heptane. As shown in Figure 8A, the diffusion coefficients of migrants (n-octadecane, n-dotriacontane or $B H T$ ) moving from polyolefins (LPE, BPE or PP) into either tributyrin or trioctanoin at either 30 or $60^{\circ} \mathrm{C}$ are almost the same as the corresponding diffusion coefficient into corn oil. The diffusion coefficients in the pure triglycerides are slightly higher than that in corn oil with a ratio of $D$ (pure triglycerides)/D(corn oil) less than 1.5 over most of the range studied. Ethanol seems to behave almost identically to the lower members of pure triglyceride in its extractions behavior as shown in Figure $8 \mathrm{~B}$. However the correlation for the data of ethanol extractions versus that of corn oil scatters more than that for triglycerides, at a factor of about 3 or less.

From the above observations, either ethanol or low members of pure triglycerides may be used successfully as food oil simulants, regardless of their dissimilarities in solvation power for the migrants, swelling power for the polymers and viscosities. 


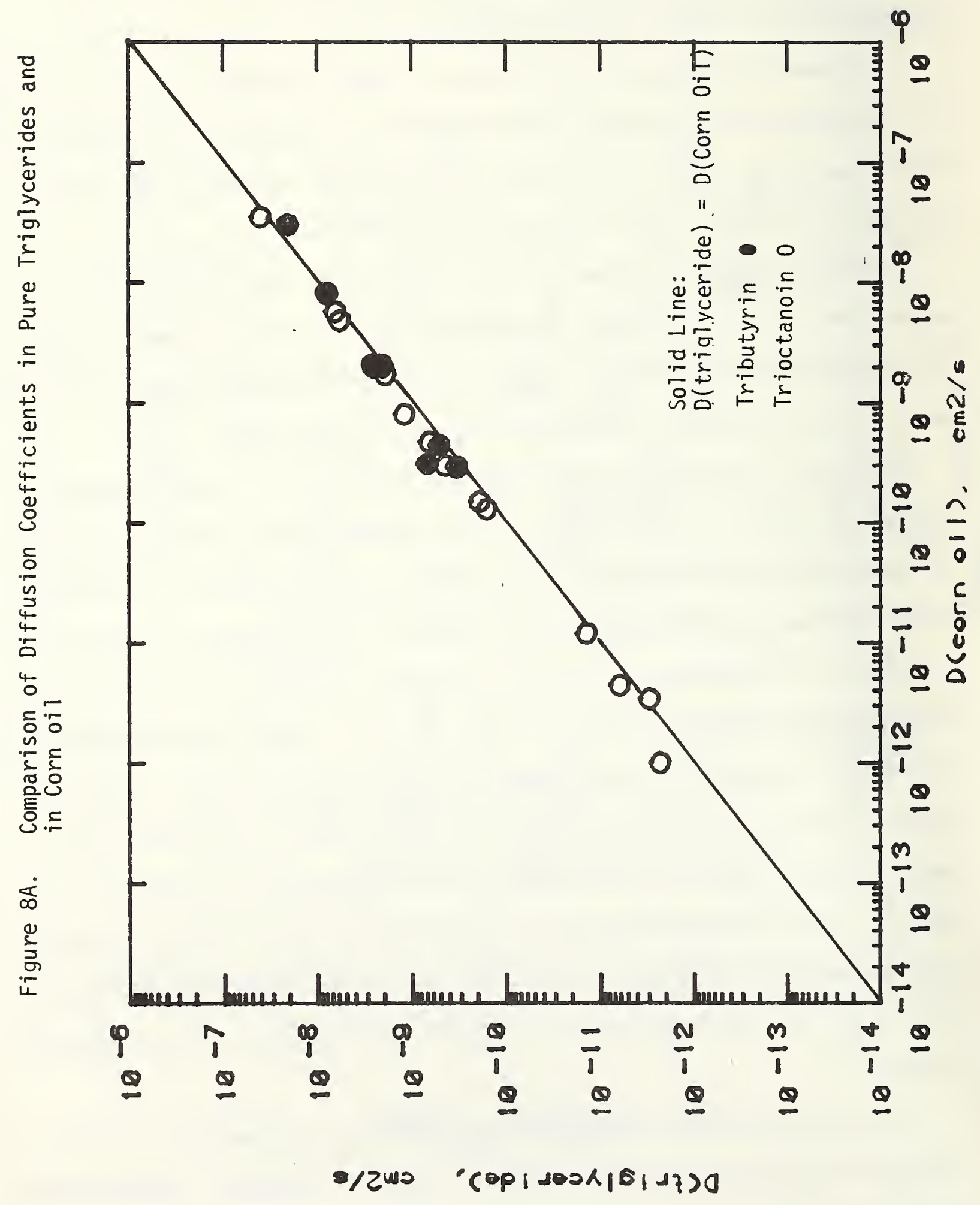




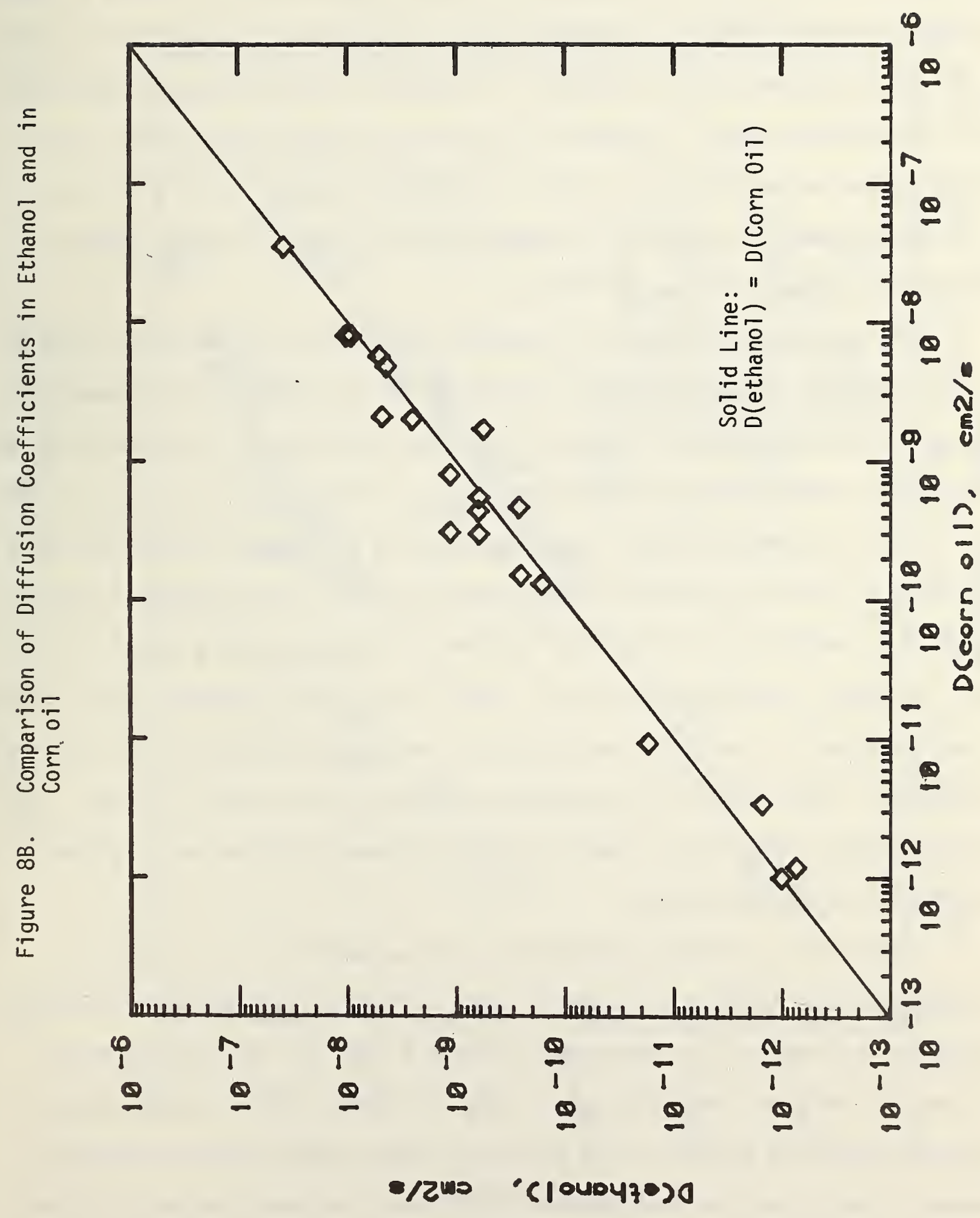


The accelerating action of $n$-heptane over that of food $0 i 1$ was long recognized. However no quantitative figures have been attached to this accelerating action. The accelerating power of $n$-heptane is clearly demonstrated in Figure 9 , where diffusion coefficients in n-heptane and in triglycerides (including corn oil) are plotted against that of ethanol for identical test samples and conditions. The accelerating power is greater at low diffusion coefficients than that at high diffusion coefficients. While at $D$ (ethanol) around $10^{-7} \mathrm{~cm}^{2} \mathrm{~s}^{-1}$ the accelerating power is about 10, the power may be increased to about 1000 at $D$ (ethanol) around $10^{-11}$ or $10^{-12} \mathrm{~cm}^{2} \mathrm{~s}^{-1}$.

The accelerating action of $n$-heptane versus that of ethanol (and that of trigiycerides), coupled with the differences in solubilities of oligomers of polyolefins, has dramatic effects on the total extractables of polyolefins. We, therefore, subjected large quantities $(50 \mathrm{~g})$ of the raw polymer stocks of SRM 1475 (LPE) and SRM 1476 (BPE) to be extracted by both $500 \mathrm{ml}$ of ethanol and $n$ heptane at $70^{\circ} \mathrm{C}$ for 160 days with occasional shaking. By assigning a minimum diffusion coefficient of about $10^{-9} \mathrm{~cm}^{2} / \mathrm{sec}$ for $n-\mathrm{C}_{32} \mathrm{H}_{66}$ and a pellet size of $0.1 \mathrm{~cm}$ radius, the effective time $T=D t / \ell^{2}=1.4$. Thus according to the generalized solution for the diffusion equation, the extraction should be at least $95 \%$ completed. The results of the 160-day extractions are listed in Table 7. For each polymer, about 6 to 8 times as much in polymer fraction was extracted by $n-$ heptane than that by ethanor.

The extracts were than subjected to gel permeation or size exclusion chromatographic analysis by Roger C. Snyder of FDA to find out their molecular weight distributions, as summarized in Table 8 and shown in Figure 10A for Tinear polyethylene SRM 1475 extracts and in Figure 10B for branched polyethylene SRM 1476 extracts. The molecular weight scales were calibrated by means of analyzing five NBS polyethylene standard reference materials, three 


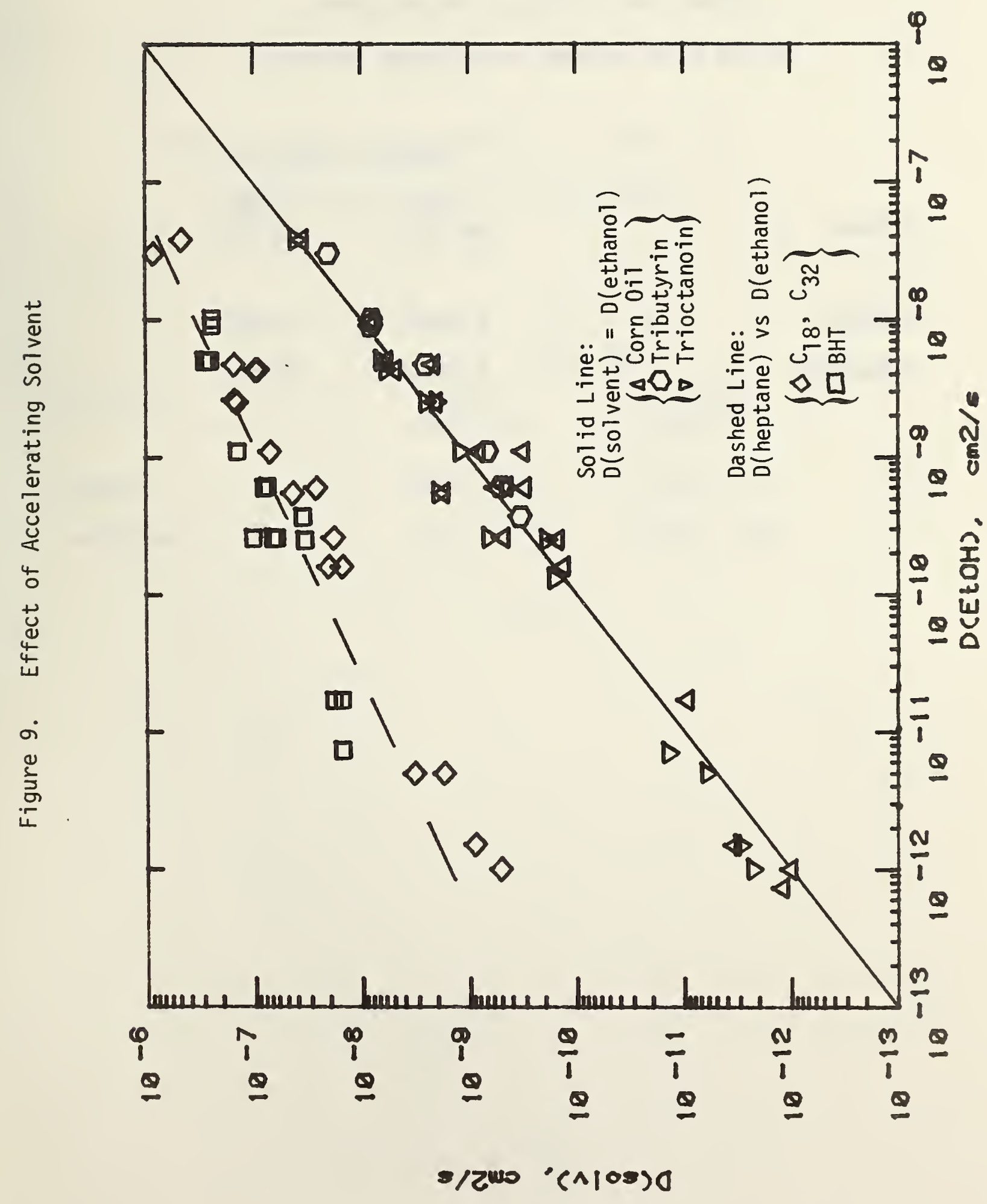


Table 7

Extraction of $50 \mathrm{~g}$ of Polyethylene

by $500 \mathrm{ml}$ of solvent at $70^{\circ} \mathrm{C}$ for 160 days

Fraction Extracted

Solvent

Ethanol

n-Heptane

\begin{tabular}{cc} 
PE & \multicolumn{1}{c}{ PE } \\
SRPA 1475 & SRM 1476 \\
& \\
0.0003 & 0.0023 \\
0.0047 & 0.018
\end{tabular}




\section{Table 8}

Molecular Weight Distributions of Ethanol and n-Heptane Extractions of Polyethylene

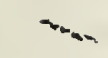

\begin{tabular}{lll} 
Solvent & \multicolumn{2}{c}{$M_{n}: M_{w}$} \\
& SRM 1475 & BPE \\
SRM 1476 \\
Ethanol & $250: 310$ & $310: 410$ \\
n-Heptane & $560: 1020$ & $620: 3000$
\end{tabular}


n-alkane from $C_{6}$ to $C_{44^{\circ}}$. The molecular weight scale for branched polyethylene is only approximately correct, however it should be sufficient for comparison purposes.

The extracts from the linear polyethylene show rather normal symmetrical distributions with peaks of molecular weight centering at about 300 for ethanol and at about 800 for $n$-heptane extracts. This type of result is expected from the practice of molecular weight fractionation by solvents of different solubility power. At the low molecular side, e.q. $\mathrm{n}-\mathrm{C}_{18} \mathrm{H}_{38}$, there is large enough solubility or even total miscibility of the oligomer fraction in both solvents, such that almost all of the low molecular weight fraction will be dissolved by the extraction processes, cf. label $A$ in Figure $10 A$ and 10B. As molecular weight gets higher, e.g. $n-C_{32} H_{66}$, the solubility in the solvent, especially ethanol, decreases which causes a partitioning of the particular component between the polymer and the extracting solvent to occur, cf. label $B$ in Figure $10 A$ and $10 B$. The partitioning of $n-C_{32} H_{66}$ has been confirmed by extractions with ethanol. The diffusion coefficient for larger molecules also drop significantly. Thus the combination of the effects of solubility, partitioning and diffusion created the shape of the molecular weight distribution curve beyond the peak molecular weight extracted.

A similar compariosn for branched polyethylene is seen in Figure 10B. The skewed curves are most likely due to the incorrectness of the size versus molecular weight calibration until one could identify the individual branched Jow molecular weight hydrocarbon species. The peak of apparent molecular weight extracted is about 350 for ethanol and about 3200 for $n$-heptane extracts. Therefore, n-heptane can not only accelerate the diffusion process but also remove high molecular species of oligomers that may be hardly detectable in ethanol or triglyceride extracts. 


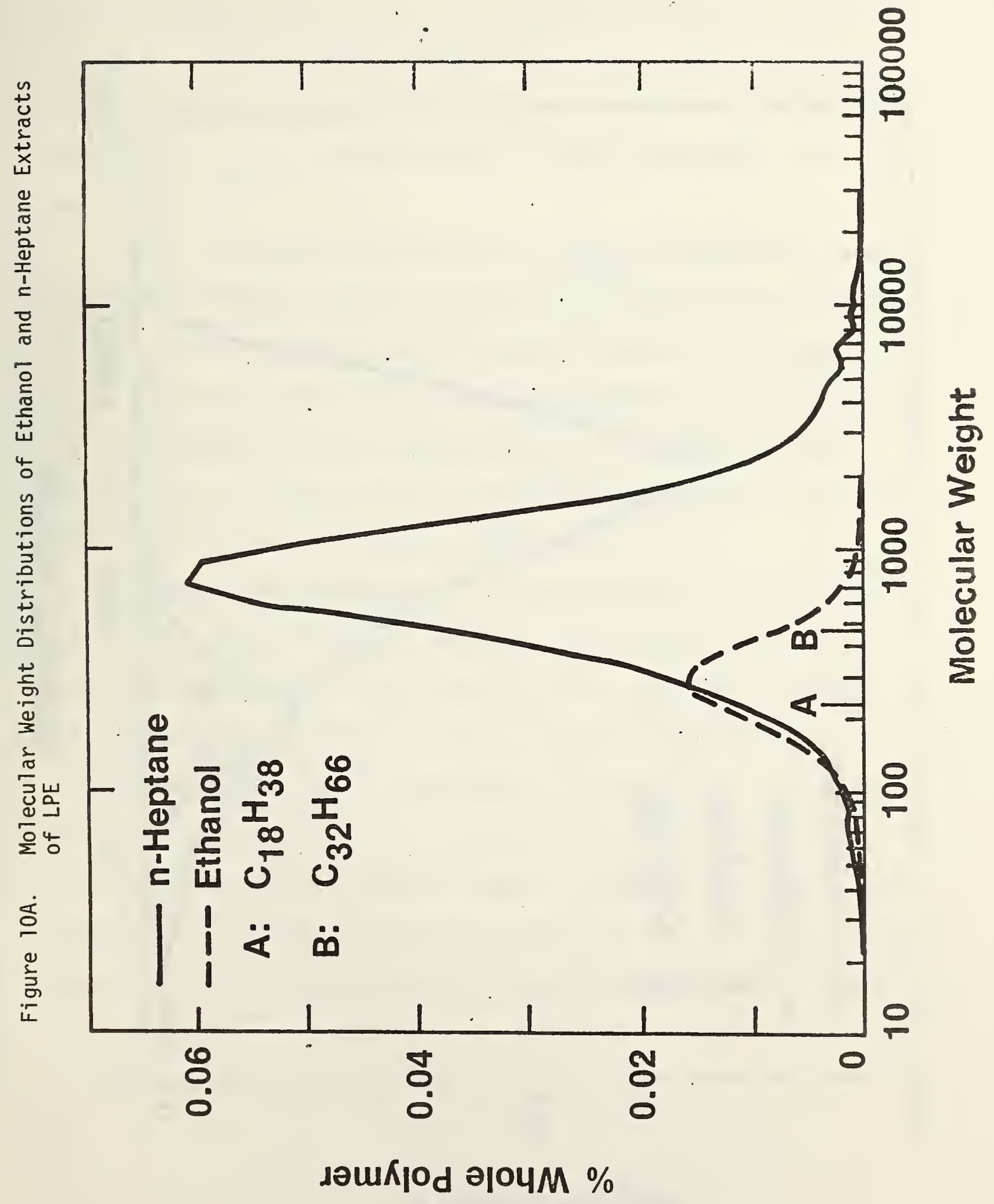




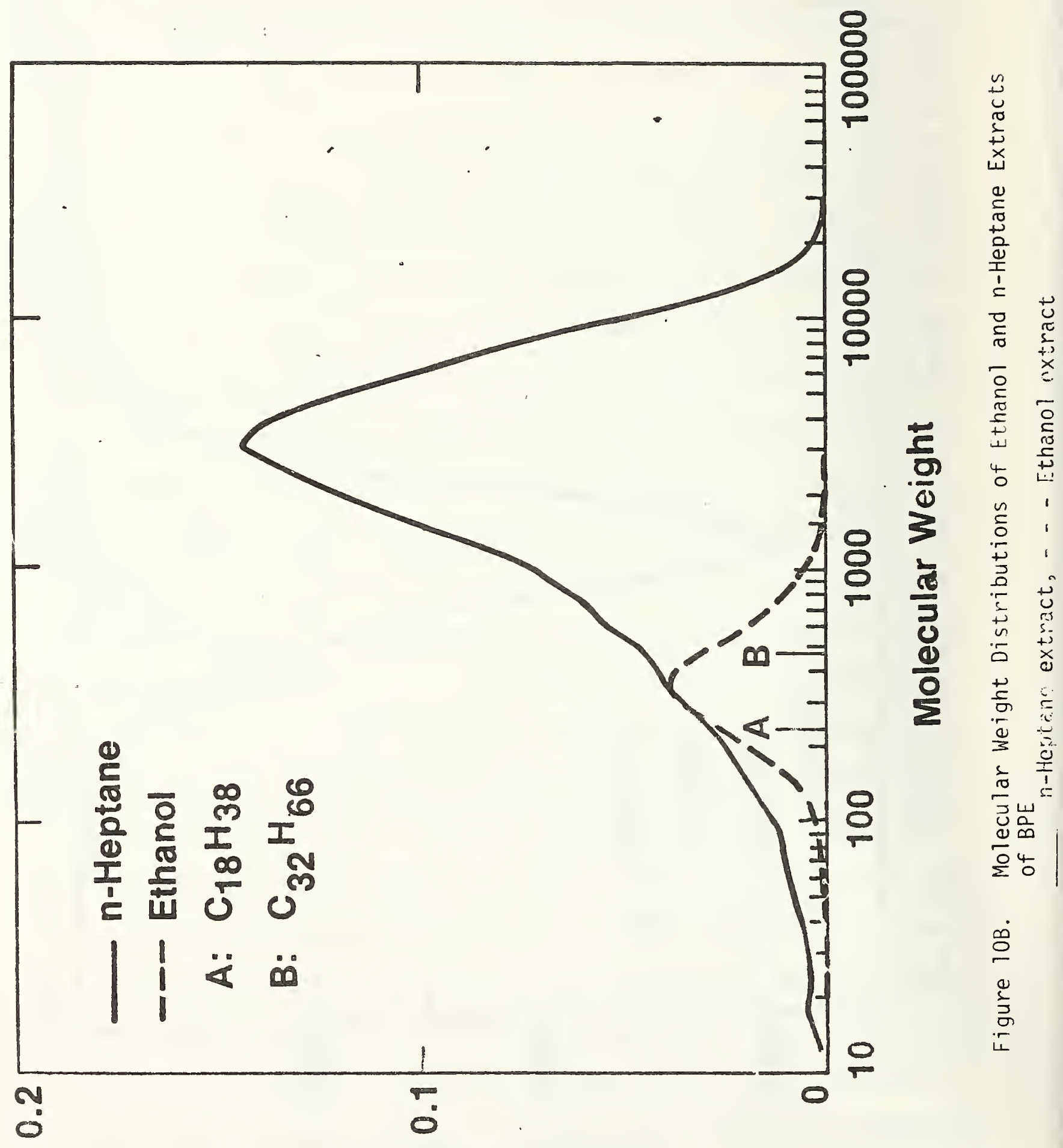

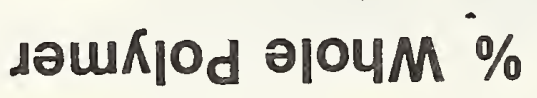


In all coses studied, the maximum reduction is chariging of uase polymer from BPE to LPE or PP is by a factor of around 30 as shown in Figure 11. The mean effect throughout the entire range of diffusion coefficients from $10^{-14}$ to $10^{-6} \mathrm{~cm}^{2} \mathrm{~s}^{-1}$ is a factor of 6 (dashed line), or $D_{\mathrm{BPE}} \cong 6 \mathrm{D}_{\mathrm{LPE}, \mathrm{PP}}$. No observation is beyond a factor of 5 from this mean correlation.

Finer correlations are possible by specifying the temperature and the migrant involved. However, the change is greater for higher temperatures. For oligomeric migrants, constant factors $\left(D_{\mathrm{LPE}, \mathrm{PP}} / \mathrm{D}_{\mathrm{BPE}}=1 / 2\right.$ at $30^{\circ} \mathrm{C}$ and $1 / 6$ at $60{ }^{\circ} \mathrm{C}$ ) may be assigned independent of solvents used. For the migration of BHT, although the, effect is greater at higher temperatures, no constant factors may be assigned. A general trend of great effect at lower diffusion coefficients prevails.

Therefore, the influences of different polyolefins on the diffusion coefficient cannot be directly related to the crystallinities of the polyolefins alone. The crystallinities of BPE, LPE, and PP are about 55,75 , and $90 \%$, respectively. Since the diffusion coefficient, as a first approximation, may be proportional to the amorphous content or the mean free volume of the entire system, the effect of different polyolefins on migration is relatively small. Slight perturbations in the temperature coefficient or activation energy, as well as in solubilities, may produce just as large an effect as due to amorphous content alone. 


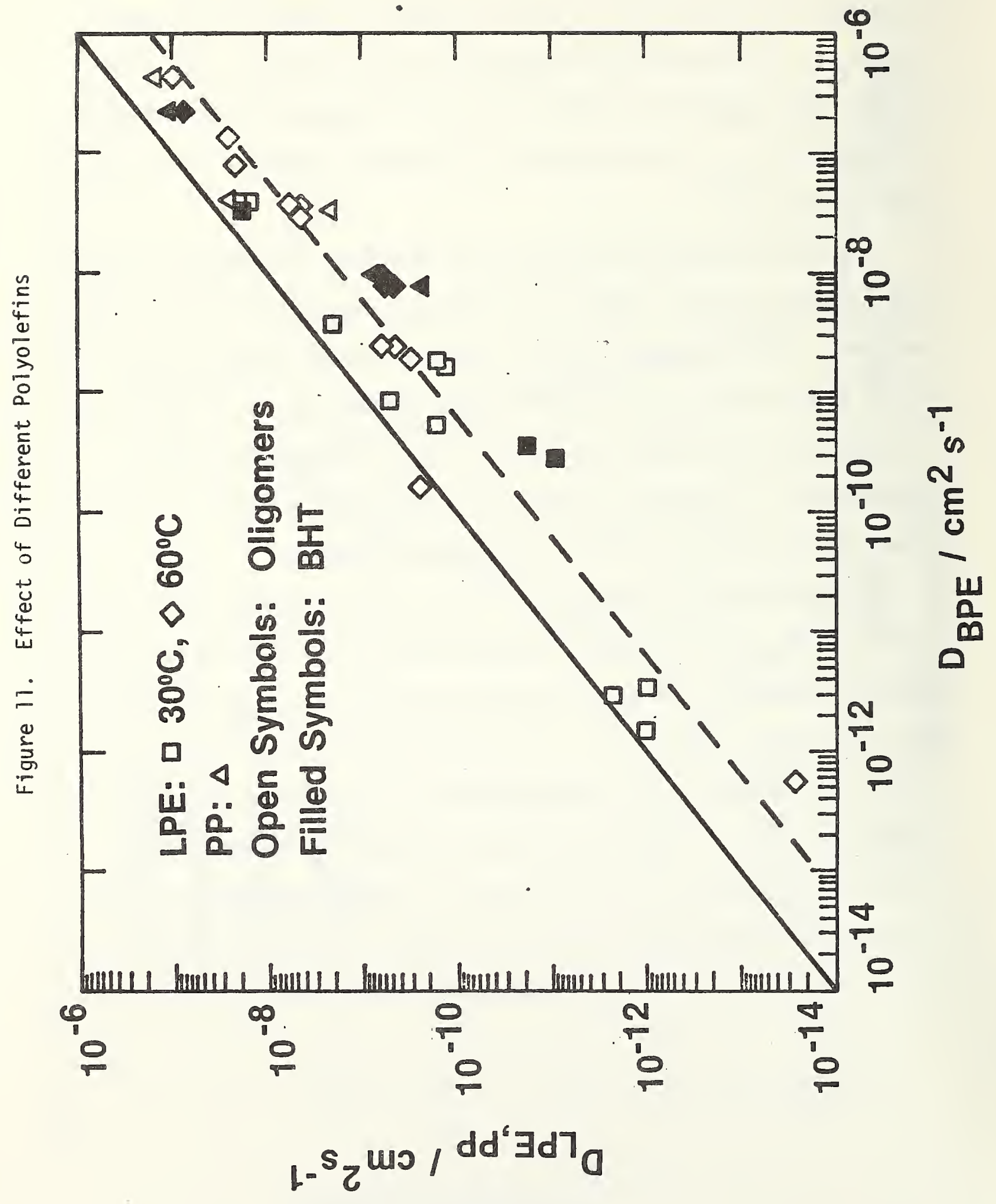




\section{Effect of Migrants}

As expected, the diffusion coefficient of oligomers of higher molecular weight species will be less than that of lower molecular species, as shown in Figure 12, where diffusion coefficients of $n$ dotriacontane is plotted versus that of $n$-octadecane. There can be a change in the diffusion coefficients by a factor of 500. Quantitative assessment of the effect of changing in molecular weight of oligomers is rather difficult because of other factors and perturbations. Nevertheless, the general trend of having greater effect at lower diffusion coefficient prevails. The trend of the effect is greater at $30^{\circ} \mathrm{C}$ than at $60{ }^{\circ} \mathrm{C}$. However, the effect is greater in BPE than in LPE and that the trend of the effect seems greater for $n$-heptane extractions than that for ethanol and triglyceride extractions.

As seen in Figure 13, the extraction behavior of BHT and n-octadecane are almost identical when $n$-heptane is used as the extracting solvent. However, the diffusion coefficient of BHT is generally about 4 to 10 times lower than that of n-octadecane when ethanol or trigylcerides are used as extracting solvents. 


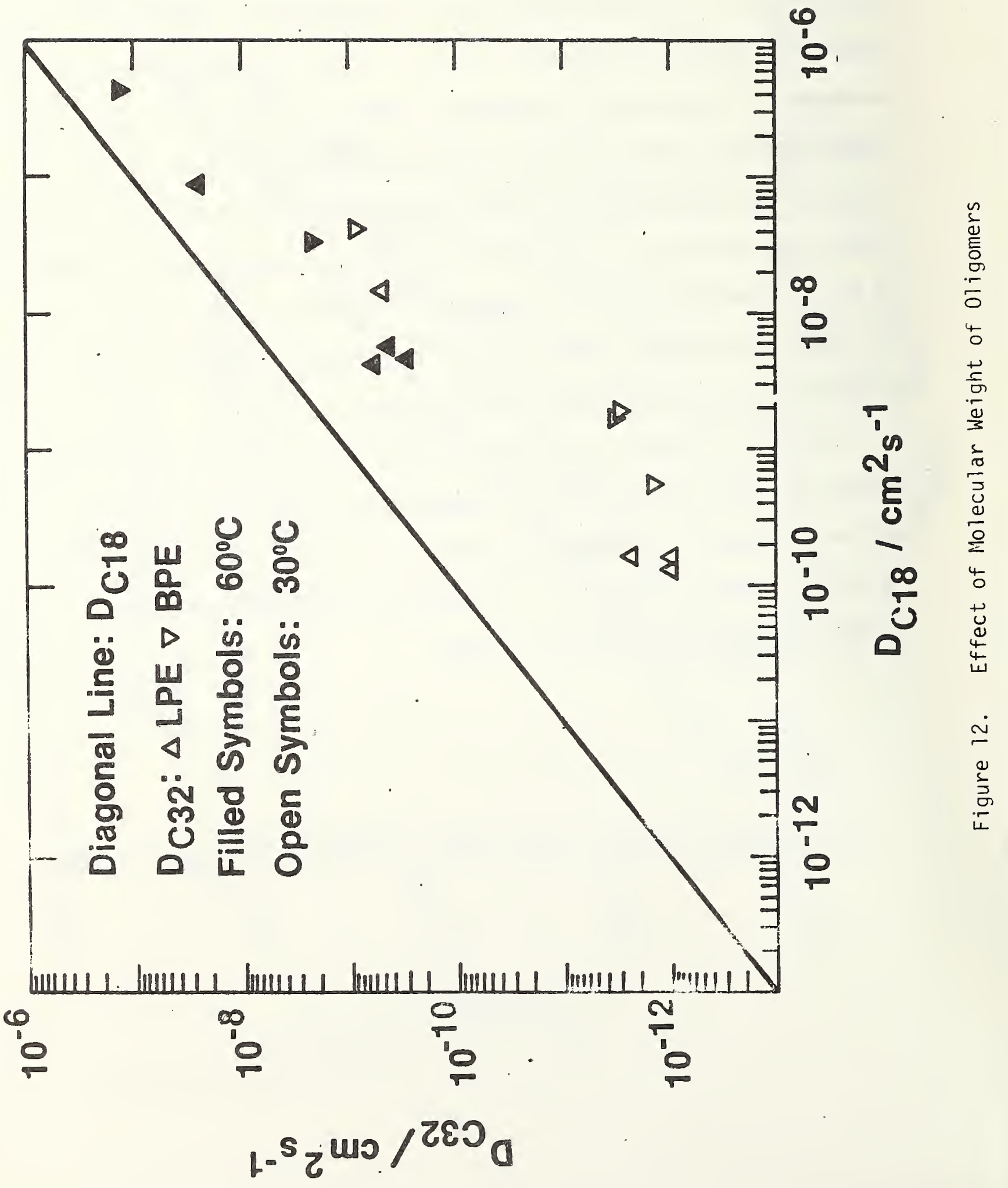




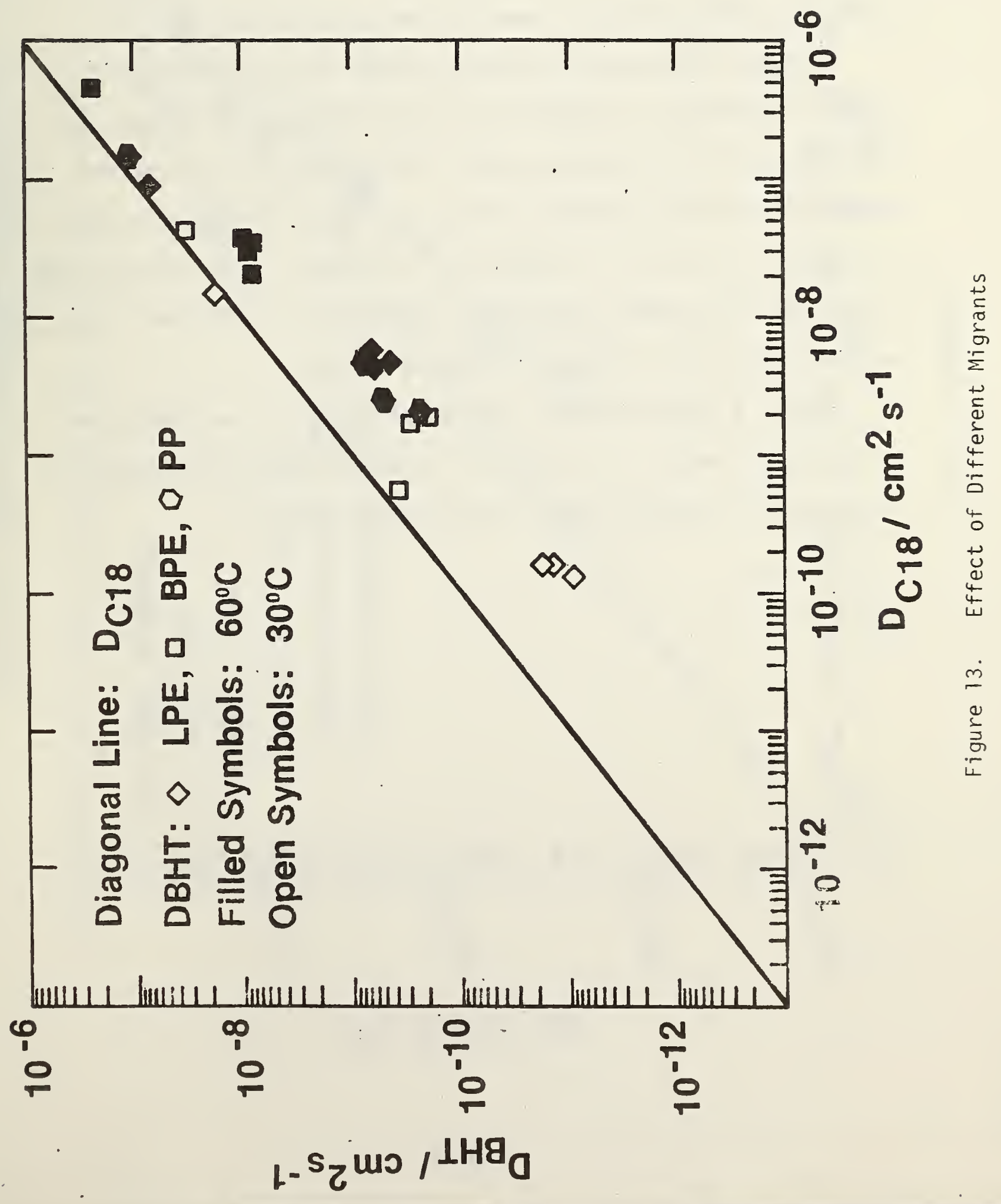




\section{Effect of Migrant Concentrations}

For relatively insoluble migrants in polyolefin, such as BHT, no significant difference in diffusion coefficient was observed as a function of the migrant concentration.

01 igomers may be viewed as either a plasticizer or low molecular weight swelling solvents for the polymer. Therefore, their contents are expected to have marked influence on the migration behavior.

Figure 14 shows the influence of added $n-\mathrm{C}_{18} \mathrm{H}_{38}$ concentration toward the diffusion coefficients of $n-\mathrm{C}_{18} \mathrm{H}_{38}$ from LPE into various solvents at different temperatures. For changes of concentration between $0.01 \%$ and $1 \%$ loading of $n-\mathrm{C}_{18} \mathrm{H}_{38}$, the diffusion coefficients change by a factor of 1 to about 30. For change of concentration from $1 \%$ to 5 or $10 \%$ loading (saturated), the diffusion coefficient changes by a factor of 5 to 20 , with hydrocarbon as solvents.

Again, a general trend of greater influence at lower diffusion coefficient prevails. The effect of oligomer concentration seems to disappear at diffusion coefficient around $10^{-6} \mathrm{~cm}^{2} \mathrm{~s}^{-1}$. 


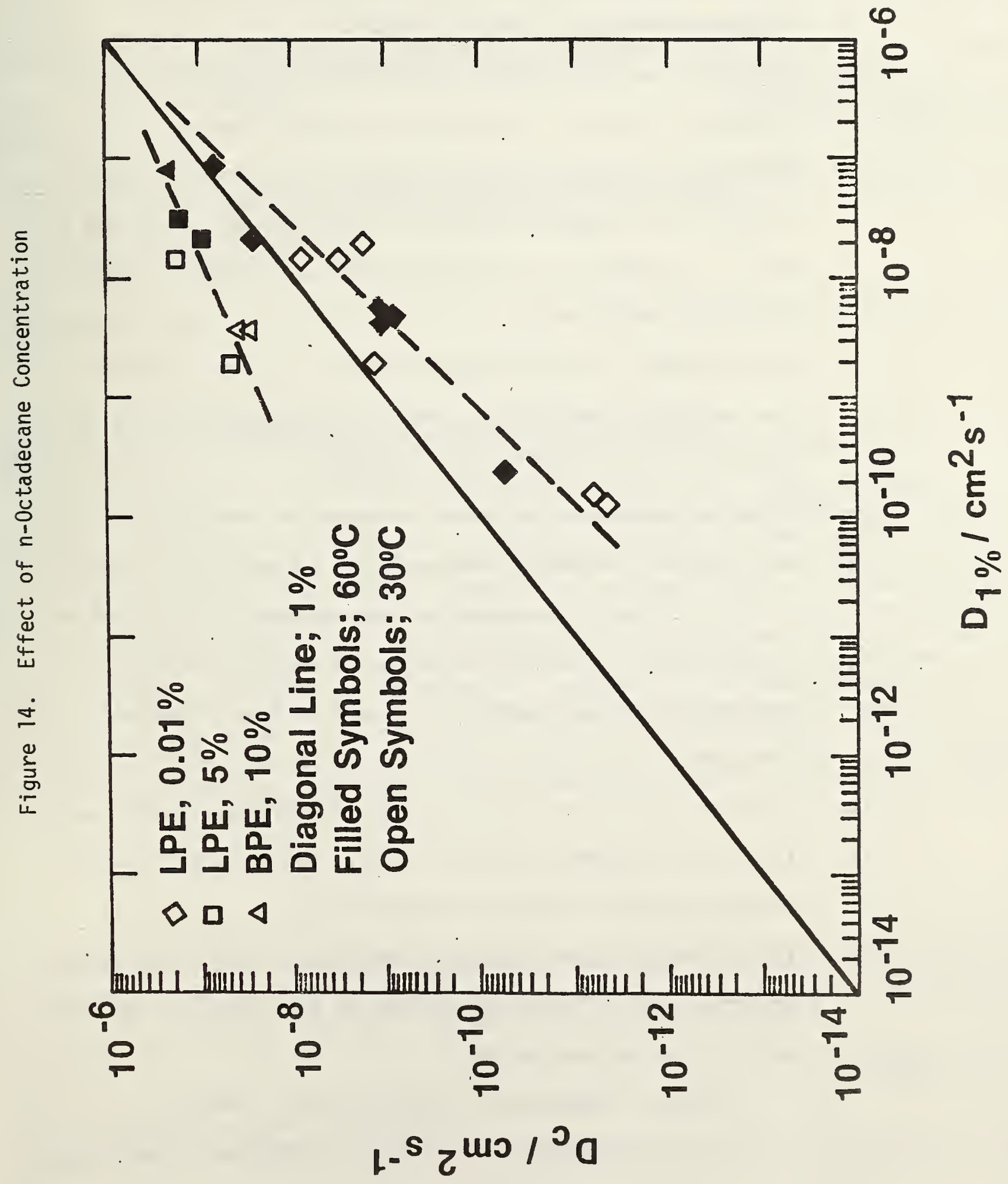


- Maximum amount of extractables depends on the original migrant concentration in the polymer, the solubilities of the migrant in solvent and in polymer or the partition coefficient.

- Migration behavior is mainly Fickian.

- Diffusion coefficient may be increased by absorption of the solvent or swelling of the polymer by the solvent.

- Activation energies for the change of diffusion coefficient as a function of temperature generally lies between 40 to $200 \mathrm{~kJ}$ $\mathrm{mol}^{-1}$. In general, the activation energy is lower at higher diffusion coefficients.

- Lower members of pure triglycerides may be used to simulate the extraction behavior of corn oil in oligomers and antioxidants, such as BHT, from polyolefins.

- Anhydrous ethanol may also be considered as food-oil simulant.

- Accelerating action of $n$-heptane over that of food-oils or oil simulants is more pronounced at lower diffusion coefficients.

- n-Heptane will remove higher molecular weight fraction of oligomers which may hardly be detected in food oil or $0 i 1$ simulants.

- Diffusion coefficient increases with increased oligomer content.

- Increase of amorphous content in polyolefin produces a corresponding change in diffusion coefficient.

- The following is the ranking of importance of the parameters effecting the diffusion coefficient of a migrant from polymer matrix into surrounding media.

Solvent > Temperature (1 imited range) > Swelling $>$ Migrant Concentration > Amorphous content. 
4. TITLE AND SUBTITLE

Models for the Migration of Low Molecular Weight Additives in Polyolefins FDA Annual Report for the Period October 1, 1979 to September 30, 1980

5. AUTHOR(S)

L. E. Simith, S. S. Chang, F. L. McCracken, G. A. Senich, F. W. Wang

6. PERFORMING ORGANIZATION (If joint or other than NBS, see instructions)

NATIONAL BUREAU OF STANDARDS

DEPARTMENT OF COMMERCE

WASHINGTON, D.C. 20234

7. ContracU/Grant No.

FDA 224-77-2443

8. Type of Report \& Period Covered

Annua1, $10 / 1 / 79-$

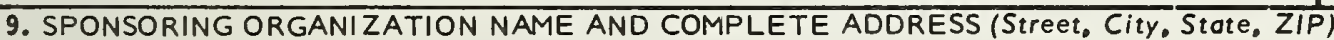
$9 / 30 / 80$

Food and Drug Administration

Rockville, MD 20857

10. SUPPLEMENTARY NOTES

Document describes a computer program: SF-185, FIPS Software Summary, is attached.

11. ABSTRACT (A 200-word or less factual summary of most significant information. If document includes a significant bibliography or literature survey. mention it here)

Food Packaging is an important encounter in the daily life. The low molecular weight components in the packaging materials may migrate into the foods. This program, sponsored by the Bureau of Foods of the Food and Drug Administration, tends to provide theoretical models, reliable data base, and methodology to study the migration phenomene and to provide reasonable worst-case estimates for the concentrations of the indirect additives in food. In this annual report we present a relationship of diffusion coefficients of gaseous diffusants in polyolefins based on free volume theory, procedures and results of inverse gas chromatography for migrant-polymer interaction parameters and diffusivities of oligomers in polyethylene, methods and preliminary results of spectrofluorimetry on the migration of antioxidants in polyethylene, results and correlations of extraction experiments with radioactive labeled migrants. It was found that anhydrous ethanol and lower numbers of pure triglycerides can successfully simulate the extractive behavior of food oils. The accelerating action of $n$-heptane over that of the food oil is quantified.

12. KEY WORDS (Six to twelve entries: alphabetical order; capitalize only proper names; and separate key words by semicolons) antioxidants; diffusion; food packaging; spectrofluorimetry; inverse gas chromatography migration; oil simulants; oligomers; radioactive tracer; polyolefins

13. AVAILABILITY

8 Unlimited

14. NO. OF PRINTED PAGES

$\square$ For Official Distribution. Do Not Release to NTIS

$\square$ Order From Superintendent of Documents, U.S. Government Printing Office, Washington, D.C. 20402.

15. Price

Order From National Technical Information Service (NTIS), Springfield, VA. 2216I 


